\title{
On Besov Spaces and Sobolev Spaces of Generalized Functions Defined on a General Region
}

\author{
By \\ Tosinobu Muramatu
}

\section{$\S 1$. Introduction}

In this paper we shall take a new approach to the theory of Besov spaces (or Lipschitz spaces) and Sobolev spaces of generalized functions or distributions defined on an open set with the cone property. These function spaces on the whole $n$-space $\mathbb{R}^{n}$ has been extensively studied by many authors, see e.g. [1], [2], [6], [7], [12], [22], [31], [32], [33]. However, it seems that theory of the spaces on an open subset $\Omega$ of $\mathbb{R}^{n}$ has not been completed up to now. To study it we shall employ the integral operator of the form

$$
\begin{aligned}
& \int_{0}^{a} t^{\sigma} d_{*} t \int K(t, x,-z, x+t z) f(x+t z) d z, \quad \text { or } \\
& \int_{0}^{a} t^{\sigma} d_{*} t \int K(t, x,-z, x+t z) u(t, x+t z) d z,
\end{aligned}
$$

where $\mathrm{K}$ satisfies appropriate conditions stated below. A representation of generalized functions by means of the integrals as above, stated in Theorem 1 and proved by an elementary calculus, make it possible to discuss the various problem, such as extension to the whole $n$-space, imbedding and determination of the interpolation space and the dual space, without any help of approximation of generalized function by smooth functions. Another key result in this report is Theorem 2, a characterization of the function spaces by means of "regularization", which was suggested by $\mathrm{H}$. Komatsu to the author. Taibleson [32] gave an analogue to this for Besov spaces on $\mathbb{R}^{n}$, which is an $n$-dimensional version of the result by

Received January 25, 1973. 
Hardy-Littlewood [11], and E. M. Stein proved the fact that $L^{p}\left(\mathbb{R}^{n}\right)$-norm, $1<p<\infty$, is equivalent to that of the Littlewood-Paley $g$-function which is very similar to our results for Sobolev spaces, see for example [31]. But their theory depends on the Poisson integral, while ours on the convolution with smooth functions having compact support. Our theory for the interpolation space of Sobolev spaces leans on the $L^{p}$-boundedness result for a class of pseudo-differential operators, instead of the theory for maximal function, $g$-function and harmonic functions.

Let us explain our notations:

Notations. $\quad x=\left(x_{1}, \ldots, x_{n}\right), y, z$ will denote points in Euclidean $n$-space $\mathbb{R}^{n}$, and $\alpha=\left(\alpha_{1}, \ldots, \alpha_{n}\right), \beta, \gamma$ multi-indices of non-negative integers.

$$
\begin{aligned}
& |\alpha|=\alpha_{1}+\cdots+\alpha_{n}, \alpha !=\alpha_{1} ! \cdots \alpha_{n} !,|x|^{2}=x_{1}^{2}+\cdots+x_{n}^{2}, x^{\alpha}=x_{1}^{\alpha_{1}} \cdots x_{n}^{\alpha_{n}}, \\
& D_{j}=\partial / \partial x_{j}, D^{\alpha}=D_{1}^{\alpha_{1}} \cdots D_{n}^{\alpha_{n}} .
\end{aligned}
$$

We say $\alpha \geqq \beta$ if $\alpha_{j} \geqq \beta_{j}, i=1, \ldots, n$.

$$
\left(\begin{array}{l}
\alpha \\
\beta
\end{array}\right)=\frac{\alpha !}{(\alpha-\beta) ! \beta !} \quad \text { for } 0 \leqq \beta \leqq \alpha,
$$

and is equal to zero otherwise. By $I$ we will denote the interval $[0, a]$ with $0<a<t_{0}$. For $t \in I \quad d_{*} t=t^{-1} d t$, for $y \in \mathbb{R}^{n} \quad d_{*} y=|y|^{-n} d y$, where $d y=d y_{1} \cdots d y_{n}$, the usual Lebesgue measure. $\mathbb{R}^{+}$is the set of positive real numbers. Moreover we will make use of the following abbreviations:

$$
\begin{aligned}
& K^{(\alpha, \gamma, \beta)}(t, x, z, y)=D_{x}^{\alpha} D_{z}^{\gamma} D_{y}^{\beta} K(t, x, z, y), \\
& u^{(\alpha)}(t, x)=D_{x}^{\alpha} u(t, x), \Omega_{j, y}=\bigcap_{k=0}^{j}(\Omega-k y) .
\end{aligned}
$$

$X, Y$ will denote Banach spaces, and $L^{p}(M, d \mu ; X)$ the space of $X$ valued $L^{p}$ functions on a measure space $(M, d \mu)$.

$$
\begin{aligned}
L^{p}(\Omega ; X)=L^{p}(\Omega, d x, X), \quad L_{*}^{p}\left(\mathbb{R}^{n} ; X\right) & =L^{p}\left(\mathbb{R}^{n}, d_{*} x ; X\right), \\
\text { and } L_{*}^{p}(I ; X) & =L^{p}\left(\mathbb{I}, d_{*} t ; X\right) .
\end{aligned}
$$

$C^{\infty}(\Omega ; X)=\mathscr{E}(\Omega ; X)$ is the spaces of $X$-valued infinitely differentiable functions, $C_{0}^{\infty}(\Omega ; X)=\mathscr{D}(\Omega ; X)$ the space of all $\varphi \in C^{\infty}(\Omega ; X)$ with compact 
support contained in $\Omega$, and $\mathscr{B}^{\infty}(\Omega ; X)$ is the space of $f \in C^{\infty}(\Omega ; X)$, whose derivatives of all order are bounded on $\Omega$. On the other hand, $\mathscr{D}^{\prime}(\Omega ; X)$ is the space of $X$-valued generalized functions or distributions on $\Omega$, and $\mathscr{E}^{\prime}(\Omega ; X)$ is the space of those with compact support contained in $\Omega$. $L^{p}(\Omega)=L^{p}(\Omega ; \mathbb{C}), C^{\infty}(\Omega)=C^{\infty}(\Omega ; \mathbb{C})$, etc.

$(X, Y)_{\theta, p}$ is the mean interpolation space due to Lions-Peetre [17], and $[X, Y]_{\theta}$ is the complex interpolation space due to Calderón $[8]$ and Lions $[15]$.

Now, we shall give the definition of the spaces and a few remarks about our results.

Definition. As usual, for a non-negative integer $m W_{p}^{m}(\Omega ; X)$, $1 \leqq p \leqq \infty$, will denote the set of all $L^{p}(\Omega ; X)$ functions whose generalized derivatives of order up to $m$ belong to $L^{p}(\Omega ; X)$. For a negative inieger $-m, W_{p}^{-m}(\Omega ; X)$ will denote the set of all $X$-valued generalized functions $f$ on $\Omega$ with

$$
f(x)=\sum_{|\alpha| \leqq m} D_{x}^{\alpha} f_{\alpha}(x), \quad f_{\alpha}(x) \in \mathbb{L}^{p}(\Omega ; X) .
$$

The norm of the space is

$$
\|f\|_{W_{p}^{-m}(\Omega ; X)}=\inf \sum_{|\alpha| \leqq m}\left\|f_{\alpha}(x)\right\|_{L^{p}(\Omega ; X)},
$$

the infimum being taken over all $\left\{f_{\alpha}\right\}$ satisfying (1.3). $W_{p}^{\infty}=\bigcap_{k} W_{p}^{k}$, $W_{p}^{-\infty}=\bigcup_{k} W_{p}^{k}$.

The space $B_{p, q}^{\sigma}(\Omega ; X)$, $\sigma$ real, $1 \leqq p, q \leqq \infty$, is defined as follows: For the case $\sigma=k+\theta$, where $0<\theta<1, k$ is a non-negative integer, $B_{p, q}^{\sigma}(\Omega ; X)$ is the set of functions $f$ such that $f \in \mathbb{W}_{p}^{k}(\Omega ; X)$ and

$$
\left\|\left[|| f^{(c)}(x+y)-f^{(u)}(x) \|_{L^{\rho}\left(\Omega_{1, y} ; X\right)}|y|^{-\theta^{-}}\right]\right\|_{L_{*}^{q}\left(\mu^{n}{ }^{n}\right)}
$$

is finite for any multi-index $\alpha$ with $|\alpha|=k$.

The norm is the sum of the norm of $W_{p}^{k}$ and the above semi-norms for all $|\alpha|=k$. For the case $\sigma=k+1$, where $k$ is a non-negative integer, the Besov space $B_{p, q}^{\sigma}(\Omega ; X)$ is defined analogously except that (1.5) is replaced by

$$
\|\left[\left\|f^{(\alpha)}(x+2 y)-2 f^{(\alpha)}(x+y)+f^{(\alpha)}(x)\right\|_{L^{p}\left(\Omega_{2, y} ; X\right)}|y|^{-1^{-}} \|_{L_{*}^{q}\left(\mathbb{R}^{n}\right)} .\right.
$$


For the case $\sigma=-k+\theta$, where $0<\theta \leqq 1$ and $k$ is a positive integer, $B_{p, q}^{\sigma}(\Omega ; X)$ is the space of generalized functions $f$ such that

$$
f(x)=\sum_{|\alpha| \leqq k} D_{x}^{\alpha} f_{\alpha}(x), \quad f_{\alpha} \in B_{p, q}^{\theta}(\Omega ; X),
$$

and the norm is

$$
\inf \sum_{|\alpha| \leqq k}\left\|f_{\alpha}\right\|_{B_{p, q}^{\theta}(\Omega ; X)}
$$

Finally, the space $H_{p}^{\sigma}(\Omega ; X)$ is

$$
H_{p}^{\sigma}(\Omega ; X)=\left[W_{p}^{k}(\Omega ; X), W_{p}^{k+1}(\Omega ; X)\right]_{\theta},
$$

if $\sigma=k+\theta, 0<\theta<1$, and $k$ is an integer; $H_{p}^{\sigma}(\Omega ; X)=W_{p}^{\sigma}(\Omega ; X)$ if $\sigma=k$ is an integer.

In the following of the paper we shall always assume that $\Omega$ is an open set with the cone property, that is, there exist a function $\Psi(x) \in \mathscr{B}^{\infty}\left(\mathbb{R}^{n} ; \mathbb{R}^{n}\right)$ and a number $t_{0}>0$ such that for any $x \in \Omega$ and $0 \leqq t<t_{0} x+t \Psi(x)+t B$ is contained in $\Omega$, where $B$ is the unit ball in $\mathbb{R}^{n}$ (in regard of this point, see $[20] \S 1$ ). Replacing $\Psi(x)$ by $(\eta+\varepsilon) \Psi(x)$, $\eta \geqq 1 \varepsilon>0$, and $t_{0}$ by $t_{0}(\eta+\varepsilon)^{-1}$, if necessary, we may assume that for any $x \in \bar{\Omega}$, the closure of $\Omega$, and $0<t<t_{0} x+t \Psi(x)+t \eta B$ is contained in $\Omega$. In connection with this we shall denote by $A(t, x)$ the closure of the set of all points $z$ such that there exist $t_{1}, t_{2}, \ldots, t_{m} \geqq 0, z^{(1)}, \ldots, z^{(m)} \in B$ with $t=t_{1}+\cdots+t_{m}, x_{1}=x+t_{1} \Psi(x)+t_{1} z^{(1)}, x_{2}=x_{1}+t_{2} \Psi\left(x_{1}\right)+t_{2} z^{(2)}, \ldots$, and $x_{m}=x+t z=x_{m-1}+t_{m} \Psi\left(x_{m-1}\right)+t_{m} z^{(m)}$. It is evident that, $x+t A(t, x) \subset \Omega$ if $x \in \bar{\Omega}, 0<t<t_{0}$ or if $x \in \bar{\Omega}, 0<t<t_{0}$, and that

$$
A(t, x) \subset b B \quad \text { for } 0<t<t_{0},
$$

where $b=\sup |\Psi(x)|+1$. Also by definition we find that

$$
w \in A\left(t_{1}, x\right), t z-t_{1} w \in\left(t-t_{1}\right) A\left(t-t_{1}, x+t_{1} w\right)
$$

with $0 \leqq t_{1}<t$ implies $z \in A(t, x)$.

It was proved that for any open set $\Omega$ with the cone property the space $B_{p, q}^{\sigma}(\Omega), 0<\sigma<2$, is identical with the set of functions $f \in L^{p}(\Omega)$ such that

$$
\left\|\left[\|f(x+2 y)-2 f(x+y)+f(x)\|_{L^{p}\left(\Omega_{2, y}\right)}|y|^{-\sigma}\right]\right\|_{L_{*}^{q}\left(\boldsymbol{R}^{n}\right)}
$$


is finite. (see [18]). The same fact is valid for the $X$-valued case.

Sometimes the space $H_{p}^{\sigma}\left(\mathbb{R}^{n}\right)$ is defined by Fourier transform, (see e.g. [8], [15], [27]) and $H_{p}^{\sigma}(\Omega)$ the restriction of $H_{p}^{\sigma}\left(\mathbb{R}^{n}\right)$ to $\Omega$. However, from Theorem 6 this space coincides with $H_{p}^{\sigma}(\Omega)$ defined above for the case $1<p<\infty$.

Next, let us introduce the space of generalized functions with "zero boundary value":

Definition. Let $\mathscr{F}(\Omega ; X)$ be a space of $X$-valued generalized functions. By $\mathscr{F}_{\bar{\Omega}}\left(\mathbb{R}^{n} ; X\right)$ we denote the set of $f \in \mathscr{F}\left(\mathbb{R}^{n} ; X\right)$ with support contained in $\bar{\Omega}$.

Then, from Theorem 5 Corollary $C_{0}^{\infty}(\Omega ; X)$ is dense in $B_{p, q, \bar{\alpha}}^{\sigma}\left({ }^{n}{ }^{n} ; X\right)$ for $1 \leqq p, q \leqq \infty$ and dense in $H_{p, \Omega}^{\sigma}\left(\mathbb{R}^{n} ; X\right)$ for $1<p<\infty$. In addition, for $1<p, q<\infty, B_{p, q}^{\sigma}(\Omega)$ and $B_{p^{\prime}, q^{\prime}, \Omega}^{-\sigma}$ are dual each other. $H_{p}^{\sigma}(\Omega)$ and $H_{p^{\prime}, \Omega}^{-\sigma}$ are also dual each other (Theorem 11). Hence our negative norm is a generalization of the one due to P. D. Lax [14].

Finally let us state the conditions imposed on the kernel $K$ of the operator (1.1).

Definition. We say $K(t, x, z, y) \in \mathscr{K}_{0}$ if it is a $\mathscr{B}^{\infty}\left(\mathbb{R}^{n} \times \mathbb{R}^{n} \times \mathbb{R}^{n}\right)$ valued continuous function of $0 \leqq t \leqq t_{0}$ and if its support regarded as a function of $z$ is contained in $-A(t, x)$ for any fixed $t, x, y . K \in \mathscr{K}_{j}$, where $j$ is a positive integer, if

$$
K(t, x, z, y)=\sum_{|\alpha|=j} K_{\alpha}^{(0, \alpha, 0)}(t, x, z, y)
$$

for some $K_{\alpha} \in \mathscr{K}_{0},|\alpha|=j . \quad K \in \overline{\mathscr{K}}_{j}$, if

$$
K(t, x, z, y)=\sum_{|\alpha|=j-1} K_{\alpha}^{(0, \alpha, 0)}(t, x, z, y)
$$

for some $K_{\alpha} \in \mathscr{K}_{0}$ with $\int K_{\alpha}(t, x, z, y) d z=0,|\alpha|=j-1$.

It is obvious that $\mathscr{K}_{j} \subset \overline{\mathscr{K}}_{j}$.

\section{§2. Calculus of Generalized Fumetions}

Definition 2.1. By $\mathscr{M}^{q}(I \times \Omega ; X),\left(\right.$ or $\left.\mathscr{U}_{0}^{q}(I \times \bar{Q} ; X)\right), 1 \leqq q \leqq \infty$, we 
denote the set of all $\mathscr{D}^{\prime}(\mathscr{\Omega} ; X)$-valued (or $\mathscr{D}_{\bar{\Omega}}\left(\mathbb{R}^{n} ; X\right)$-valued) functions $u(t, x)$ of $t \in \mathbb{I}$ such that for every compact subset $C$ in $\Omega$ (or in $\mathbb{R}^{n}$ ) there exist a non-negative integer $k$ and functions

$$
\begin{aligned}
& u_{\alpha}(t, x) \in L_{*}^{q}\left(\mathbb{I} ; L_{\text {loc }}^{1}(\Omega ; X)\right),|\alpha| \leqq k, \\
& \left(\text { or } u_{\alpha}(t, x) \in L_{*}^{q}\left(I ; L_{\text {loc }}^{1}\left(\mathbb{P}^{n} ; X\right)\right)\right),
\end{aligned}
$$

satisfying

$$
u(t, x)=\sum_{|\alpha| \leqq k} D_{x}^{\alpha} u_{\alpha}(t, x) \text { on } \mathbb{C} .
$$

EXmple $\mathbb{Z}_{\mathbb{X}} \mathbb{1}$. If $\rho \in \mathbb{L}_{*}^{q}(I), f \in \mathscr{D}^{\prime}(\Omega ; X)$, (see $[26]$, note that every Banach space is a complete $(D F)$-space), then $\rho(t) f(x) \in \mathscr{M}^{q}(\mathbb{I} \times \Omega ; X)$.

Example 2.2。 Let $1 \leqq p, q \leqq \infty$. Then

$$
\begin{aligned}
& L^{p}\left(\Omega ; L_{*}^{q}(I ; X)\right) \subset \mathscr{M}^{q}(I \times \Omega ; X), \\
& L_{*}^{q}\left(I ; L^{p}(\Omega ; X)\right) \subset \mathscr{M}^{q}(I \times \Omega ; X) .
\end{aligned}
$$

Lemping 2. I。 (I) Let $\mathbb{K}(t, x, z, y)$ belong to $\mathscr{K}_{m}$. Assume that $\rho(t) t^{m} \in \mathbb{L}_{*}^{1}(\mathbb{I})$. Then

(a) for any $\varphi \in \mathscr{E}(\Omega)$ the integral

$$
\begin{aligned}
\varphi(t, x) & =\int \mathbb{K}(t, x,-z, x+t z) \varphi(x+t z) d z \\
& =t^{-n} \int \mathbb{K}\left(t, x, \frac{x-y}{t}, y\right) \varphi(y) d y
\end{aligned}
$$

is well defind and

$$
\varphi(t, x) \in \mathscr{E}(\Omega) \text { for every fixed } t,
$$

$$
\varphi(t, x) \rightarrow k(x) \varphi(x) \text { in } \mathscr{E}(\Omega) \text { as } t \rightarrow 0
$$

where

$$
k(x)=\int \mathbb{K}(0, x, z, x) d z
$$

$$
\int_{0}^{a} \varphi(t, \gamma) \rho(t) d_{*} t, \text { is connergent in } \mathscr{E}(\Omega) \text {. }
$$


In particular, in the case where $\Omega=\mathbb{R}^{n}$ the same facts are also valid when $\mathscr{E}(\Omega)$ is replaced by $\mathscr{D}\left(\mathbb{R}^{n}\right)$.

(b) For any $\psi \in \mathscr{D}(\Omega)$ the integral

$$
\begin{aligned}
\psi(t, x)=\int K(t, & x+t z, z, x) \psi(x+t z) d z \\
& =t^{n} \int K\left(t, y, \frac{y-x}{t}, x\right) \psi(y) d y
\end{aligned}
$$

is well defined and

$$
\phi(t, x) \in \mathscr{D}(\Omega) \text { for every fixed } t
$$

$$
\psi(t, x) \rightarrow k(x) \psi(x) \text { in } \mathscr{D}(\Omega) \text { as } t \rightarrow 0 \text {, }
$$

$$
\int_{0}^{a} \psi(t, x) \rho(t) d_{*} t \text { is convergent in } \mathscr{D}(\Omega) .
$$

(II) Let $X$ be a Banach space and let $K(t, x, z, y)$ belong to $\mathscr{K}_{0}$. Then

(a) for $f \in \mathscr{D}^{\prime}(\Omega ; X)$ and $t>0$

$$
\begin{aligned}
U(t, x) & =\int K(t, x,-z, x+t z) f(x+t z) d z, \\
& =t^{-n}<K\left(t, x, \frac{x-y}{t}, y\right), f(y)>_{y} .
\end{aligned}
$$

$\left(<,>\right.$ denotes the duality between $\mathscr{D}(\Omega)$ and $\left.\mathscr{D}^{\prime}(\Omega ; X)\right)$ is well defined and

$$
U(t, x) \in C^{\infty}(\Omega ; X) \text { for every fixed } t>0,
$$

$$
U(t, x) \rightarrow k(x) f(x) \text { in } \mathscr{D}^{\prime}(\Omega ; X) \text { as } t \rightarrow 0,
$$

(b) for any $g \in \mathscr{D} \frac{\prime}{\bar{a}}\left(\mathbb{R}^{n} ; X\right)$, and $t>0$

$$
\begin{aligned}
V(t, x) & =\int K(t, x+t z, z, x) g(x+t z) d z \\
& =t^{-n}<K\left(t, y, \frac{y-x}{t}, x\right), g(y)>_{y}
\end{aligned}
$$

is well defined and 
(i)

$$
V(t, x) \in C^{\infty}\left(\mathbb{R}^{n} ; X\right), \operatorname{supp}(V(t, x)) \subset \Omega
$$

(In particular, if $g \in \mathscr{E}^{\prime}(\Omega ; X)$ then $V(t, x) \in C_{0}^{\infty}(\Omega ; X)$ ) for every fixed $t>0$,

$$
V(t, x) \rightarrow k(x) g(x) \text { in } \mathscr{D}^{\prime}\left(\mathbb{R}^{n} ; X\right) \text { as } t \rightarrow 0 \text {. }
$$

Remark. It is convenient to write the duality between distributions and differentiable functions by the integral. Hence we make use of this convention in the following if no confusion occurs.

Proof. (I) (a) The integral $\varphi(t, x)$ is well defined, since for fixed $t$ and $x \in \Omega$

$$
\operatorname{supp}\left[K\left(t, x, \frac{x-y}{t}, y\right)\right] \subset x+t A(t, x)
$$

Part (i) is evident. To prove part (ii) we observe that for any compact subset $C$ in $\Omega$ the set

$$
I \times\{(x,-z, y) \mid x \in C, z \in A(t, x), y=x+t z, 0 \leqq t \leqq a\}
$$

is compact, therefore, $D_{x}^{\alpha}\{K(t, x,-z, x+t z) \varphi(x+t z)\}$ converges to $D_{x}^{\alpha}\{K(0, x,-z, x) \varphi(x)\}$ uniformly on the above set as $t \rightarrow 0$. This implies that $D_{x}^{\alpha} \varphi(t, x) \rightarrow D_{x}^{\alpha}\{k(x) \varphi(x)\}$ uniformly on $C$ as $t \rightarrow 0$. Hence $\varphi(t, x) \rightarrow$ $k(x) \varphi(x)$ in $\mathscr{E}(\Omega)$. In the case where $\Omega=\mathbb{R}^{n}$ and $\varphi \in \mathscr{D}\left(\mathbb{R}^{n}\right)$, using the fact

$$
\operatorname{supp}[\varphi(t, x)] \subset \operatorname{supp}(\varphi)+t b B
$$

as is readily seen, we find that

$$
\varphi(t, x) \rightarrow k(x) \varphi(x) \text { in } \mathscr{D}\left(\mathbb{R}^{n}\right)
$$

In order to establish part (iii), we first consider the case $m=0$. Let $\varepsilon$ be a positive number, and set

$$
\varphi_{\varepsilon}(x)=\int_{\varepsilon}^{a} \varphi(t, x) \rho(t) d_{*} t
$$

Since for any compact set $C$ in $\Omega$ the set 


$$
A=\{y \mid y \in x+t A(t, x), x \in C, 0 \leqq t \leqq a\}(\subset \Omega)
$$

is compact, it follows that

$$
\begin{aligned}
\left|D^{\alpha} \varphi_{\varepsilon}(x)-D^{\alpha} \varphi_{\delta}(x)\right| & \leqq \int_{\delta}^{\varepsilon} \rho(t) d_{*} t \int\left|D_{x}^{a}\{K(t, x,-z, x+t z) \varphi(x+t z)\}\right| d z \\
& \leqq C \sup _{\beta \leqq \alpha} \sup _{A}\left|D^{\beta} \varphi(y)\right| \int_{\delta}^{\varepsilon} \rho(t) d_{*} t \rightarrow 0
\end{aligned}
$$

as $\varepsilon, \delta \rightarrow 0$. Therefore, $\varphi_{\varepsilon}(x)$ is convergent in $\mathscr{E}(\Omega)$. Part (iii) for the case $m \geqq 1$ follows immediately from that for the case $m=0$ with the aid of the following identity (cf. Cor. 2);

$$
\begin{aligned}
\varphi(t, x) & -\sum_{|a|=m} \int K_{a}^{(1), n,(1)}(t, x, \cdots z, x+t z) \varphi(x+t z) d z, \\
& =\iota^{m} \sum_{|\alpha|=m} \sum_{\beta}\left(\begin{array}{c}
\alpha \\
\beta
\end{array}\right) \int K_{a}^{(0,0, a-\beta)}(t, x,-z, x+t z) \varphi^{(\beta)}(x+t z) d z .
\end{aligned}
$$

(I) (b). Since $\varphi \in \mathscr{D}\left(\mathcal{R}^{n}\right)$, it is evident that $\varphi(t, x) \in C^{\infty}(\Omega)$. Let $C$ be the support of $\varphi$. Then the set $A$ defined by (2.1) is compact and contains the support of $\phi(t, x)$ for every $t$. Hence the fact $\phi(t, x) \rightarrow k(x) \psi(x)$ in $\mathscr{D}(\Omega)$ as $t \rightarrow 0$ follows from the fact

$$
D_{x}^{\alpha}\{K(t, x+t z, z, x) \psi(x+t z)\} \rightarrow D_{x}^{\alpha}\{K(0, x, \approx, x) \psi(x)\}
$$

uniformly on $A \times b B$ since $I \times A \times b B$ is compact. Part (iii) can be verified in the same manner as (I) (a) part (iii).

(II) (a). Since for fixed $t>0, x \in \Omega$ the function $K(t, x,(x-y) / t, y)$ of $y \in \Omega$ belongs to $\mathscr{D}(\Omega), U(t, x)$ is well defined. Part (i) is immediately verified by the definition of generalized functions. Let us consider part (ii). From Fubini's theorem it follows that for any $\psi \in \mathscr{D}(\Omega)$

$$
\begin{aligned}
\int U(t, x) \psi(x) d x & =\left\langle i^{-n} K\left(t, x, \frac{x-y}{t}, y\right) \psi(x), f(y) \otimes 1_{x}>_{(x, y)}\right. \\
& =<\int t^{-n} K\left(t, x, \frac{x-y}{t}, y\right) \psi(x) d x, f(y)>_{y} \\
& =\left\langle\psi(t, y), f(y)>_{y}\right.
\end{aligned}
$$


whence, applying (I) (b) (ii), we obtain

$$
\int U(t, x) \psi(x) d x \rightarrow<k(y) \psi(y), f(y)>=<\psi(y), k(y) f(y)>
$$

as $t \rightarrow 0$.

(II) (b). Since for any fixed $t>0, x$, the function $K(t, y,(y-x) / t, x)$ of $y$ belongs to $\mathscr{D}\left(\mathbb{R}^{n}\right), V(t, x)$ is well defined. It is easily find that

$$
\operatorname{supp} V(t, x) \subset\{x \mid x=y+t A(t, y), y \in \operatorname{supp}(g)\} \subset \Omega \text {. }
$$

The remainder parts of (b) can be shown similarly.

Corollary 1. With the same assumptions and notations as in the lemma, we have

$$
\int U(t, x) \psi(x) d x=\langle\psi(t, x), f(x)>
$$

for $f \in \mathscr{D}^{\prime}(\Omega ; X), \phi \in \mathscr{D}(\Omega)$, and

$$
\int V(t, x) \varphi(x) d x=<\varphi(t, x), g(x)>
$$

for either $g \in \mathscr{E}^{\prime}(\Omega ; X), \varphi \in \mathscr{E}(\Omega)$ or $g \in \mathscr{D}_{\bar{\Omega}}^{\prime}\left(\mathbb{R}^{n} ; X\right), \varphi \in \mathscr{D}\left(\mathbb{R}^{n}\right)$.

Corollary 2. With the some assumptions and notations as in the lemma, we have

$$
\begin{aligned}
& D_{x}^{\alpha} U(t, x)=\sum_{\beta}\left(\begin{array}{l}
\alpha \\
\beta
\end{array}\right) t^{-|\beta|} \int K^{(\alpha-\beta, \beta, 0)}(t, x,-z, x+t z) f(x+t z) d z \\
& D_{x}^{\alpha} V(\iota, x)=\sum_{\beta}\left(\begin{array}{l}
\alpha \\
\beta
\end{array}\right)(-t)^{-|\beta|} \int K^{(0, \beta, \alpha-\beta)}(t, x+t z, z, x) g(x+t z) d z \\
& \int K^{(0, \alpha, 0)}(t, x,-z, x+t z) f(x+t z) d z \\
& \quad=t^{|\alpha|} \sum_{\beta} \int\left(\begin{array}{l}
\alpha \\
\beta
\end{array}\right) K^{(0,0, \alpha-\beta)}(t, x,-z, x+t z) f^{(\beta)}(x+t z) d z \\
& \int K^{(0, \alpha, 0)}(t, x+t z, z, x) g(x+t z) d z \\
& =(-l)^{|a|} \sum_{\beta}\left(\begin{array}{l}
\alpha \\
\beta
\end{array}\right) \int K^{(a-\beta, 0,0)}(t, x+t z, z, x) g_{g}^{(\beta)}(x+t z) d z .
\end{aligned}
$$


Proof. It is easy to find that

$$
\begin{aligned}
D_{x}^{\alpha} U(t, x) & =t^{-n}<D_{x}^{\alpha}\left\{K\left(t, x, \frac{x-y}{t}, y\right)\right\}, f(y)> \\
& =\sum_{\beta}\left(\begin{array}{c}
\alpha \\
\beta
\end{array}\right) t^{-n-|\beta|}<K^{(\alpha-\beta, \beta, 0)}\left(t, x, \frac{x-y}{t}, y\right), f(y)>,
\end{aligned}
$$

and that

$$
\begin{aligned}
D_{x}^{\alpha} V(t, x) & =t^{-n}<D_{x}^{\alpha}\left\{K\left(t, y, \frac{y-x}{t}, x\right)\right\}, g(y)> \\
& =\sum_{\beta}\left(\begin{array}{l}
\alpha \\
\beta
\end{array}\right) t^{-n-|\beta|}(-1)^{|\beta|}<K^{(0, \beta, \alpha-\beta)}\left(t, y, \frac{y-x}{t}, x\right) f(y)>.
\end{aligned}
$$

From the identity

$$
\sum_{\beta}(-1)^{|\alpha-\beta|}\left(\begin{array}{l}
\alpha \\
\beta
\end{array}\right)\left(\begin{array}{l}
\beta \\
\gamma
\end{array}\right)= \begin{cases}1 & \text { for } \alpha=\gamma \\
0 & \text { for } \alpha \neq \gamma,\end{cases}
$$

it follows that

$$
\begin{aligned}
& \sum_{\beta} t^{-n}\left(\begin{array}{l}
\alpha \\
\beta
\end{array}\right)<K^{(0,0, \alpha-\beta)}\left(t, x, \frac{x-y}{t}, y\right), f^{(\beta)}(y)> \\
= & \sum_{\beta} t^{-n}(-1)^{|\beta|}\left(\begin{array}{l}
\alpha \\
\beta
\end{array}\right)<D_{y}^{\beta}\left\{K^{(0,0, \alpha-\beta)}\left(t, x, \frac{x-y}{t}, y\right)\right\}, f(y)> \\
= & \sum_{\gamma} \sum_{\beta} t^{-n}(-1)^{|\beta-\gamma|}\left(\begin{array}{l}
\alpha \\
\beta
\end{array}\right)\left(\begin{array}{l}
\beta \\
\gamma
\end{array}\right) t^{-|\gamma|}<K^{(0, \gamma, \alpha-\gamma)}\left(t, x, \frac{x-y}{t}, y\right), f(y)> \\
= & t^{-n-|\alpha|}<K^{(0, \alpha, 0)}\left(t, x, \frac{x-y}{t}, y\right), f(y)>.
\end{aligned}
$$

Similarly, we have

$$
\begin{aligned}
\sum_{\beta} t^{\prime \prime \prime}\left(\begin{array}{l}
\alpha \\
\beta
\end{array}\right) & <K^{(\alpha-\beta, 0,0)}\left(t, y, \frac{y-x}{t}, x\right), g^{(\beta)}(y)> \\
& =t^{-n-|a|}(-1)^{|a|}<\mathbb{K}^{(0, \alpha, 0)}\left(i, y, \frac{y-x}{t}, x\right), g(y)>.
\end{aligned}
$$

Liprollary 3. Assume that 


$$
K(t, x, z, y)=\sum_{|\alpha|=m} K_{\alpha}^{(0, \alpha, 0)}(t, x, z, y),
$$

where $K_{\alpha} \in \mathscr{K}_{0}$, and set for every $|\beta| \leqq m$

$$
\begin{aligned}
& \widehat{K}_{\beta}(t, x, z, y)=\sum_{|\alpha|=m}\left(\begin{array}{l}
\alpha \\
\beta
\end{array}\right)(-1)^{|\alpha-\beta|} K_{\alpha}^{(\alpha-\beta, 0,0)}(t, x, z, y) \\
& \tilde{K}_{\beta}(t, x, z, y)=\sum_{|\alpha|=m}\left(\begin{array}{l}
\alpha \\
\beta
\end{array}\right)(-1)^{|\alpha-\beta|} K_{\alpha}^{(0,0, \alpha-\beta)}(t, x, z, y)
\end{aligned}
$$

(Note that $K_{\beta}=\hat{K}_{\beta}=\tilde{K}_{\beta}$ for $|\beta|=m$ ).

Then for $f \in \mathscr{D}^{\prime}(\Omega ; X)$ and $g \in \mathscr{D}_{\bar{\Omega}}^{\prime}\left(\mathbb{R}^{n} ; X\right)$

$$
\begin{aligned}
& \begin{aligned}
U(t, x)=\int K(t, x, & -z, x+t z) f(x+t z) d z \\
& =t^{m} \sum_{|\beta| \leqq m} D_{x}^{\beta} \int \hat{K}_{\beta}(t, x,-z, x+t z) f(x+\iota z) d z
\end{aligned} \\
& V(t, x)=\int K(t, x+t z, z, x) g(x+t z) d z \\
& =(-t)^{m} \sum_{|\beta| \leqq m} D_{x}^{\beta} \int \tilde{K}_{\beta}(t, x+t z, z, x) g(x+t z) d z .
\end{aligned}
$$

Proof. From Corollary 2 and from (2.2) it follows that

$$
\begin{aligned}
& t^{m} \sum_{\beta} D_{x}^{\beta} \int \hat{K}_{\beta}(t, x,-z, x+t z) f(x+t z) d z \\
= & \sum_{\beta} \sum_{\gamma} t^{m-|\gamma|}\left(\begin{array}{c}
\beta \\
\gamma
\end{array}\right) \int \hat{K}_{\beta}^{(\beta-\gamma, \gamma, 0)}(t, x,-z, x+t z) f(x+t z) d z, \\
= & \sum_{\gamma} \sum_{\beta} \sum_{|\alpha|=m} t^{m-|\gamma|}\left(\begin{array}{l}
\beta \\
\gamma
\end{array}\right)\left(\begin{array}{l}
\alpha \\
\beta
\end{array}\right)(-1)^{|\alpha-\beta|} \int K_{\alpha}^{(\alpha-\gamma, \gamma, 0)}(t, x,-z, x+t z) f(x+t z) d z \\
= & \int_{|\alpha|=m} K_{\alpha}^{(0, \alpha, 0)}(t, x,-z, x+t z) f(x+t z) d z=U(t, x) .
\end{aligned}
$$

The proof of the second identity goes the same way.

Lemma 2.2. Let $K(t, x, z, y) \in \mathscr{K}_{0}$.

(a) Let $u(t, x) \in \mathscr{M}^{1}(I \times \Omega ; X)$ and let

$$
U(t, x)=\int K(t, x,-z, x+t z) u(t, x+t z) d z .
$$


Then the integral $\int_{0}^{a} U(t, x) d_{*} t$ is convergent in $\mathscr{D}^{\prime}(\Omega, X)$.

(b) Let $v(t, x) \in \mathscr{M}_{0}^{1}(\Gamma \times \bar{\Omega} ; X)$ and let

$$
V(t, x)=\int K(t, x+t z, z, x) v(t, x+t z) d z
$$

Then the integral $\int_{0}^{a} V(t, x) d_{*} t$ is convergent in $\mathscr{D}_{\bar{a}}^{\prime}\left(\mathbb{R}^{n} ; X\right)$.

Proof. (a) By Lemma 2.1 Corollary 1 we get

$$
\int U(t, x) \psi(x) d x=<\psi(t, x), u(t, x)>_{x}
$$

for every $\psi \in \mathscr{D}(\Omega)$, where $\phi(t, x)$ is the function defined in Lemma 2.1 I. (b). Let $A$ be the compact set given by (2.1) where $C$ is the support of $\psi$. Then the support of $\psi(t, x), t>0$, is contained in $A$. Since $u \in \mathscr{M}^{1}$, there exists a representation

$$
u(t, x)=\sum_{|\alpha| \leqq k} D^{\alpha} u_{\alpha}(t, x), \quad u_{\alpha} \in L_{*}^{1}\left(I, L_{l o c}^{1}(\Omega)\right)
$$

on $A$, so we obtain

$$
\left\langle\psi(t, x), u(t, x)>=\sum_{|\alpha| \leqq k}(-1)^{\mid \alpha i}\left\langle\psi^{(\alpha)}(t, x), u_{\alpha}(t, x)>.\right.\right.
$$

But

$$
\begin{aligned}
\psi^{(\alpha)}(t, x) & =\sum_{\beta}\left(\begin{array}{l}
\alpha \\
\beta
\end{array}\right)(-t)^{-|\beta|} t^{-n} \int K^{(0, \beta, \alpha-\beta)}\left(t, y, \frac{y-x}{t}, x\right) \psi(y) d y \\
& =\sum_{\gamma} \sum_{\beta}\left(\begin{array}{l}
\alpha \\
\beta
\end{array}\right)\left(\begin{array}{l}
\beta \\
\gamma
\end{array}\right) t^{-n} \int K^{(\beta-\gamma, 0, \alpha-\beta)}\left(t, y, \frac{y-x}{t}, x\right) \psi^{(\gamma)}(y) d y,
\end{aligned}
$$

which gives

$$
\left|\psi^{(\alpha)}(t, x)\right| \leqq c \sup _{\alpha \geqq \beta} \sup _{x}\left|\phi^{(\beta)}(x)\right| \leqq c\|\psi\|_{k},
$$

where $c$ is independent of $t, \psi, \alpha$ and $\|\psi\|_{k}=\sup _{|\alpha| \leqq k} \sup _{x}\left|\psi^{(\alpha)}(x)\right|$. Therefore, we obtain 


$$
\begin{aligned}
\int_{\varepsilon}^{\delta} \| \sum_{|\alpha| \leqq k}<\psi^{(\alpha)}(t, x), & u_{\alpha}(t, x)>\|_{X} d_{*} t \\
& \leqq c\|\phi\|_{k} \int_{\varepsilon|\alpha| \leqq k}^{\delta} \sum_{\mid}\left\|u_{\alpha}(t, x)\right\|_{L^{1}(A ; X)} d_{*} t \rightarrow 0
\end{aligned}
$$

as $\varepsilon, \delta \rightarrow 0$. This completes the proof of part (a). (see [26] or [34]). (b). By Lemma 2.1 (II). (b) we find that the support of $V(t, x), t>0$, is contained in $\Omega$, which gives $V(t, x) \in \mathscr{E}\left(\mathbb{R}^{n}\right)$. Therefore, it will suffice to prove the convergence in $\mathscr{D}^{\prime}\left(\mathbb{R}^{n} ; X\right)$. Suppose that $\varphi(x) \in \mathscr{D}\left(\mathbb{R}^{n}\right)$ and let $\varphi(t, x)$ be the function defined in Lemma 2.1(I) (a). Then by Lemma 2.1 Corollary 1 we have

$$
\int V(t, x) \varphi(x) d x=<\varphi(t, x), v(t, x)>_{x}
$$

Combining this with the fact that the support of $\varphi(t, x), t>0$, is contained in a compact subset in $\mathbb{R}^{n}$ which is independent of $t$, we obtain

$$
\int V(t, x) \varphi(x) d x=\sum_{|\alpha| \leqq m}(-1)^{|\alpha|} \int \varphi^{(\alpha)}(t, x) v_{\alpha}(t, x) d x
$$

where $v_{\alpha} \in L_{*}^{1}\left(I ; L_{\text {loc }}^{1}\left(\mathbb{R}^{n}\right)\right)$. Hence we obtain our assertion by discussing in the same manner as part (a).

Corollary $\mathbb{1}$. Let $1 \leqq q \leqq \infty$, and let $\sigma$ be a real number. Assume that $K \in \mathscr{K}_{j}$, where $j$ is a non-negative number such that $j+\sigma \geqq 0$ when $q=1$ and $j+\sigma>0$ when $q \geqq 1$.

(a) For $u(t, x) \in \mathscr{M}^{q}(I \times \Omega ; X)$ let us set

$$
U(t, x)=\int K(t, x,-z, x+t z) u(t, x+t z) d z .
$$

Then $\int_{0}^{a} t^{\sigma} U(t, x) d_{*} t$ is convergent in $\mathscr{D}^{\prime}(\mathscr{B} ; X)$, and

$$
D_{x}^{\alpha} \int_{0}^{a} t^{\sigma} U(t, x) d_{*} t=\int_{0}^{a} t^{\sigma} D_{x}^{\alpha} U(t, x) d_{*} t
$$

(b) For $v(t, x) \in \mathscr{M}_{0}^{q}(I \times \bar{\Omega} ; X)$ let us set

$$
V(t, x)=\int K(t, x+t z, z, x) v(t, x+t z) d z
$$


Then

$$
\int_{0}^{a} t^{\sigma} V(t, x) d_{*} t \text { is convergent in } \mathscr{D}^{\prime}\left(\mathbb{R}^{n} ; X\right),
$$

whose support is contained in $\Omega$, and

$$
D_{x}^{\alpha} \int_{0}^{a} t^{\sigma} V(t, x) d_{*} t=\int_{0}^{a} t^{\sigma} D_{x}^{\alpha} V(t, x) d_{*} t
$$

Proof. From the identity

$$
\begin{aligned}
U(t, x) & =\int \sum_{|\alpha|=j} K_{\alpha}^{(0, \alpha, 0)}(t, x,-z, x+t z) u(t, x+t z) d z, \\
& =t^{j} \sum_{|\alpha|=j} \sum_{\beta}\left(\begin{array}{l}
\alpha \\
\beta
\end{array}\right) \int K_{\alpha}^{(0,0, \alpha-\beta)}(t, x,-z, x+t z) u^{(\beta)}(t, x+t z) d z,
\end{aligned}
$$

the fact that $t^{j+\sigma} u^{(\beta)}(t, x) \in \mathscr{M}^{1}(I \times \Omega)$ which is an immediate consequence of Hölder's inequality, and from the lemma the convergence of the integral follows. Since

$$
D_{x}^{\alpha} U(t, x)=\sum_{\beta}\left(\begin{array}{l}
\alpha \\
\beta
\end{array}\right) t^{-|\beta|} \int K^{(\alpha-\beta, \beta, 0)}(t, x,-z, x+t z) u(t, x+t z) d z
$$

and since $K^{(\alpha-\beta, \beta, 0)} \in \mathscr{K}_{j+|\beta|}$, it follows that

$$
\int_{0}^{a} D_{x}^{\alpha} U(t, x) d_{*} t
$$

is convergent in $\mathscr{D}^{\prime}(\Omega ; X)$. Therefore, we obtain the formula of differentiation under integral sign by letting $\varepsilon \rightarrow 0$ in the identity

$$
D_{x}^{\alpha} \int_{\varepsilon}^{a} t^{\sigma} U(t, x) d_{*} t=\int_{\varepsilon}^{a} D_{x}^{\alpha} U(t, x) d_{*} \iota
$$

The proof of part (b) goes the same way.

Corollary 2. Let $K \in \mathscr{K}_{j}, \rho(t) t^{j} \in L_{*}^{1}(I)$. Then

(a) for $f \in \mathscr{D}^{\prime}(\Omega ; X)$ and $\psi \in \mathscr{D}(\Omega)$ we have

$$
\begin{aligned}
<\psi(x), \int_{0}^{a} \rho(t) d_{*} t \int K(t, x,-z, x+t z) f(x+t z) d z> \\
\quad=<\int_{0}^{a} \rho(t) d_{*} t \int K(t, x+t z, z, x) \psi(x+t z) d z, f(x)>;
\end{aligned}
$$


(b) for $g \in \mathscr{E}^{\prime}(\Omega ; X)$ and $\varphi \in \mathscr{E}(\Omega)$ or for $g \in \mathscr{D}_{\bar{\Omega}}^{\prime}\left(\mathbb{R}^{n} ; X\right)$ and $\varphi \in \mathscr{D}\left(\mathbb{H}^{n}\right)$ we have

$$
\begin{aligned}
<\varphi(x), \int_{0}^{a} \rho(t) d_{*} t \int K(t, x+t z, z, x) g(x+t z) d z> \\
\quad=<\int_{0}^{a} \rho(t) d_{*} t \int K(t, x,-z, x+t z) \varphi(x+t z) d z, g(x)>
\end{aligned}
$$

Proof. With the aid of a limitting argument, these identities follow from Corollary 1 and Lemma 2.1 Corollary 1.

\section{§3. Integral Representatioms}

Lemma 3.1. Let $K_{1}(x, z, y) \in \mathscr{K}_{0}$ and set

$$
\begin{aligned}
& K_{m}(x, z, y)=\sum_{|\alpha|<m} \frac{1}{\alpha !} D_{z}^{\alpha}\left\{z^{\alpha} K_{1}(x, z, y)\right\}, \\
& \tilde{K}_{m}(x, z, y)=\sum_{|\alpha|=m} \frac{1}{\alpha !} D_{z}^{\alpha}\left\{z^{\alpha} K_{1}(x, z, y)\right\} .
\end{aligned}
$$

Then, for $f \in \mathscr{D}^{\prime}(\Omega ; X)$ and $g \in \mathscr{D}_{\bar{\Omega}}^{\prime}\left(\mathbb{R}^{n} ; X\right)$

$t \frac{\partial}{\partial t} \int K_{m}(x,-z, x+t z) f(x+t z) d z=-m \int \tilde{K}_{m}(x,-z, x+t z) f(x+t z) d z$,

and

$t \frac{\partial}{\partial t} \int K_{m}(x+t z, z, x) g(x+t z) d z=-m \int \tilde{K}_{m}(x+t z, z, x) g(x+t z) d z$

Proof. From the identity

$$
\begin{aligned}
& t \frac{\partial}{\partial t}\left\{t^{-n} \int K_{m}\left(x, \frac{x-y}{t}, y\right) f(y) d y\right\} \\
&=-n t^{-n} \int K_{m}\left(x, \frac{x-y}{t}, y\right) f(y) d y \\
&-t^{-n} \int \sum_{j=1}^{n} \frac{x_{j}-y_{j}}{t} \frac{\partial K_{m}}{\partial z_{j}}\left(x, \frac{x-y}{t}, y\right) f(y) d y,
\end{aligned}
$$


and the identity

$$
n K_{m}(x, z, y)+\sum_{j=1}^{n} z_{j} \frac{\partial K_{m}}{\partial z_{j}}(x, z, y)=m \tilde{K}_{m}(x, z, y)
$$

the first identity follows. In the same way we get the second identity.

Corollary 1. (Integral representation) Let us choose $\omega(z) \in C_{0}^{\infty}\left(\mathbb{R}^{n}\right)$ such that supp $\omega$ is contained in the unit ball $B$, and that its integral is equal to 1. Put

$$
\begin{gathered}
\omega_{1}(x, z)=\omega(-z-\Psi(x)), \\
\omega_{m}(x, z)=\sum_{|\alpha|<m} \frac{1}{\alpha !} D_{z}^{\alpha}\left\{z^{\alpha} \omega_{1}(x, z)\right\}, \\
M(x, z)=\sum_{|\alpha|=m} \frac{m}{\alpha !} D_{z}^{\alpha}\left\{z^{\alpha} \omega_{1}(x, z)\right\} .
\end{gathered}
$$

Then, for $f \in \mathscr{D}^{\prime}(\Omega ; X)$ and $g \in \mathscr{D}_{\bar{\Omega}}^{\prime}\left(\mathbb{R}^{n} ; X\right)$,

$$
\begin{aligned}
& f(x)=\int_{0}^{a} d_{*} t \int M(x,-z) f(x+t z) d z+U_{m}(a, x), \\
& g(x)=\int_{0}^{a} d_{*} t \int M(x+t z, z) g(x+t z) d z+V_{m}(a, x),
\end{aligned}
$$

where

$$
\begin{aligned}
& U_{m}(t, x)=\int \omega_{m}(x,-z) f(x+t z) d z \\
& V_{m}(t, x)=\int \omega_{m}(x+t z, z) g(x+t z) d z .
\end{aligned}
$$

Proof. It follows from the lemma that

$$
U_{m}(\varepsilon, x)-U_{m}(a, x)=-\int_{\varepsilon}^{a} t \frac{\partial U_{m}}{\partial t} d_{*} t=\int_{\varepsilon}^{a} d_{*} t \int M(x,-z) f(x+t z) d z .
$$

Letting $\varepsilon \rightarrow 0$, we obtain the first identity by Lemma 2.1 (II) (a), since $\int \omega_{m}(x, z) d z=1$. The second identity is proved analogously.

Corollary 2. Let $k$ and $m$ be fixed integers such that $0 \leqq k \leqq m \neq 0$, 
and let $\omega, U_{m}$, and $V_{m}$ be the same functions as in Corollary 1. Set

$$
M_{\beta}(x, z)=\sum_{|\alpha|=k} \sum_{|\gamma|=m-k}(-1)^{|\alpha-\beta|}\left(\begin{array}{l}
\alpha \\
\beta
\end{array}\right)\left(\begin{array}{l}
m \\
k
\end{array}\right)^{-1} \frac{m}{\alpha ! \gamma !} D_{x}^{\alpha-\beta} D_{z}^{\gamma} \omega_{\alpha+\gamma}(x, z)
$$

for $|\beta| \leqq k$, where

$$
\omega_{\alpha}(x, z)=z^{\alpha} \omega(-z-\Psi(x))
$$

Then, for $f \in \mathscr{D}^{\prime}(\Omega ; X)$ and $g \in \mathscr{D}_{\bar{\Omega}}^{\prime}\left(\mathbb{R}^{n} ; X\right)$

$$
\begin{gathered}
f(x)=\sum_{|\beta| \leqq k} D_{x}^{\beta} \int_{0}^{a} t^{k} d_{*} t \int M_{\beta}(x,-z) f(x+t z) d z+U_{m}(a, x) \\
g(x)=(-1)^{k} \sum_{|\alpha|=k} D_{x}^{\alpha} \int_{0}^{a} t^{k} d_{*} t \int M_{\alpha}(x+t z, z) g(x+t z) d z+V_{m}(a, x) .
\end{gathered}
$$

Proof. Since $M(x, z)=\sum_{|\alpha|=k} M_{\alpha}^{(0, \alpha)}(x, z)$, the identities follow from Corollary 1 and Lemma 2.1 Corollary 3.

Lemma 3.2. Let $m$ be a non-negative integer and let $\sigma$ be a real number. Assume that $K(t, x, z, y) \in \mathscr{K}_{m}$ and $\sigma+m>0$.

(a) If $f \in \mathscr{D}^{\prime}(\Omega ; X)$, then

$$
t^{\sigma} \int K(t, x,-z, x+t z) f(x+t z) d z \in \mathscr{M}^{1}(I \times \Omega ; X) .
$$

(b) If $g \in \mathscr{D}_{\bar{\Omega}}^{\prime}\left(\mathbb{R}^{n} ; X\right)$, then

$$
t^{\sigma} \int K(t, x+t z, z, x) g(x+t z) d z \in \mathscr{M}_{0}^{1}(I \times \bar{\Omega} ; X) .
$$

Proof. (a). Let $C$ be a compact set in $\Omega$, and let $A$ be a set defined by (2.1). Since $A$ is compact, by the theorem on local structure of distributions $([26]$ ) we obtain that $f$ is equal to a expression

$$
\sum_{|\alpha|=k} D^{\alpha} f_{\alpha}(x), \quad f_{\alpha} \in C^{0}(\Omega ; X),
$$

in a neighbourhood of $A$. Hence for $x \in C$

$$
t^{\sigma} \int K(t, x,-z, x+t z) f(x+t z) d z
$$




$$
\begin{aligned}
& =t^{\sigma-k} \sum_{|\alpha|=k} \int\left(-D_{z}\right)^{\alpha}\{K(t, x,-z, x+t z)\} f_{\alpha}(x+t z) d z, \\
& =\sum_{|\alpha|=k} \sum_{\beta}\left(\begin{array}{l}
\alpha \\
\beta
\end{array}\right)(-1)^{|\alpha-\beta|} t^{\sigma-|\beta|} \int K^{(0, \beta, a-\beta)}(t, x,-z, x+t z) f_{\alpha}(x+t z) d z .
\end{aligned}
$$

Since $K^{(0, \beta, \alpha-\beta)} \in K_{|\beta|+m}$, it is sufficient to consider the case where $f$ is continuous in a neighbourhood of $A$, and in this case the assertion follows from

$$
\begin{aligned}
t^{\sigma} \int K(t, x,-z, x & +t z) f(x+t z) d z \\
& =t^{\sigma+m} \sum_{|\beta| \leqq m} D_{x}^{\beta} \int \widehat{K}_{\beta}(t, x,-z, x+t z) f(x+t z) d z
\end{aligned}
$$

(Lemma 2.1 Corollary 3)

and

$$
\left\|\int \widehat{K}_{\beta}(t, x,-z, x+t z) f(x+t z) d z\right\|_{L^{\infty}(C ; X)} \leqq c \sup _{x \in A}\|f(x)\|_{X}
$$

The proof of part (b) goes the same way.

Lemma $3 . B_{\text {. }}$ Let $\theta>0, \sigma \geqq 0,1 \leqq p \leqq \infty$ and let $1 \leqq \xi \leqq \eta \leqq \infty$.

(i) If $U(t, x) \in \mathscr{M}^{\xi}(I \times \Omega ; X)\left(\right.$ or $\left.\mathscr{M}_{0}^{\xi}(I \times \bar{\Omega} ; X)\right)$, then

$$
\begin{array}{r}
u(t, x)=t^{\sigma} \int_{t}^{a} t^{\theta} s^{-\theta} U(s, x) d_{*} s \in \mathscr{M}^{\eta}(I \times \Omega ; X) \\
\left(\text { or } \in \mathscr{M}_{0}^{\eta}(I \times \bar{\Omega} ; X)\right) .
\end{array}
$$

In particular, the operator $U \rightarrow u$ is a bounded linear operator from $L_{*}^{\xi}(I$; $\left.L^{p}(\Omega ; X)\right)$ into $L_{*}^{\eta}\left(I ; L^{p}(\Omega ; X)\right)$, or from $L^{p}\left(\Omega ; L_{*}^{\xi}(I ; X)\right)$ into $L^{p}(\Omega$; $\left.L_{*}^{\eta}(I ; X)\right)$ with norm $\leqq a^{\sigma} / \theta$.

(ii) If $U(t, x) \in \mathscr{M}^{\xi}(I \times \Omega ; X)\left(\right.$ or $\left.\mathscr{M}_{0}^{\xi}(I \times \bar{\Omega} ; X)\right)$ then

$$
\begin{array}{r}
u(t, x)=t^{\sigma} \int_{0}^{t} t^{-\theta} s^{\theta} U(s, x) d_{*} s \in \mathscr{M}^{\eta}(I \times \Omega ; X) \\
\left(\text { or } \in \mathscr{M}_{0}^{\eta}(I \times \bar{\Omega} ; X)\right) .
\end{array}
$$

In particular, the operator $U \rightarrow u$ is a bounded linear operator from $L_{*}^{\xi}(I$; $\left.L^{p}(\Omega ; X)\right)$ into $L_{*}^{\eta}\left(I ; L^{p}(\Omega ; X)\right)$, or from $L^{p}\left(\Omega ; L_{*}^{\xi}(I ; X)\right)$ into $L^{p}\left(\Omega ; L_{*}^{\eta}\right.$ 
$(I ; X))$ with norm $\leqq a^{\sigma} / \theta$.

Proof. These facts follows from [20] Lemma 2.5, because

$$
\int_{t}^{a} t^{\theta} s^{-\theta} d_{*} s \leqq \frac{1}{\theta}, \int_{0}^{s} t^{\theta} s^{-\theta} d_{*} t=\frac{1}{\theta}, t^{\theta} s^{-\theta} \leqq 1 \text { for } t \leqq s
$$

Now we are in a position to state and to prove a new integral representation of generalized functions which is frequently used in the following discussions:

Theorem 1. (Integral Representation). Let $h, k, l, m$ be non-negative integers such that $0 \leqq h \leqq l, 0 \leqq k \leqq m, l \neq 0, m \neq 0$. Let $\omega(z)$ be a function such that $\omega(z) \in C_{0}^{\infty}$, supp $\omega \subset B, \int \omega(z) d z=1$. Set

$$
\begin{aligned}
& \omega_{\alpha}(x, z)=z^{\alpha} \omega(-z-\Psi(x)) \\
& \omega_{m}(x, z)=\sum_{|\alpha|<m} \frac{1}{\alpha !} D_{z}^{\alpha} \omega_{\alpha}(x, z) \\
& L_{\beta}(x, z)=\sum_{|\alpha|=h} \sum_{|\gamma|=l-h} \frac{l}{\alpha ! \gamma !}\left(\begin{array}{l}
l \\
h
\end{array}\right)^{-1}\left(\begin{array}{l}
\alpha \\
\beta
\end{array}\right)(-1)^{|\alpha-\beta|} D_{x}^{\alpha-\beta} D_{z}^{\gamma} \omega_{\alpha+\gamma}(x, z), \\
& M_{\beta}(x, z)=\sum_{|\alpha|=k} \sum_{|\gamma|=m-k} \frac{m}{\alpha ! \gamma !}\left(\begin{array}{l}
m \\
k
\end{array}\right)^{-1}\left(\begin{array}{l}
\alpha \\
\beta
\end{array}\right)(-1)^{|\alpha-\beta|} D_{x}^{\alpha-\beta} D_{z}^{\gamma} \omega_{\alpha+\gamma}(x, z) . \\
& L(x, z)=\sum_{|\alpha|=l} \frac{l}{\alpha !} D_{z}^{\alpha} \omega_{\alpha}(x, z)=\sum_{|\alpha|=h} D_{z}^{\alpha} L_{\alpha}(x, z), \\
& M(x, z)=\sum_{|\alpha|=m} \frac{m}{\alpha !} D_{z}^{\alpha} \omega_{\alpha}(x, z)=\sum_{|\alpha|=k} D_{z}^{\alpha} M_{\alpha}(x, z),
\end{aligned}
$$

(a) For $f \in \mathscr{D}^{\prime}(\Omega ; X),|\alpha| \leqq k,|\beta| \leqq h$, let us write

$$
\begin{aligned}
& U^{\alpha}(s, x)=\int_{\beta} \sum_{\beta}\left(\begin{array}{l}
\alpha \\
\beta
\end{array}\right) s^{k-|\beta|} L^{(\alpha-\beta, \beta)}(x,-w) f(x+s w) d w \\
& u^{\alpha}(t, x)=\int_{t}^{a} t^{k} s^{-k} U^{\alpha}(s, x) d_{*} s
\end{aligned}
$$




$$
\begin{aligned}
& U_{\beta}(s, x)=\int L_{\beta}(x,-w) f(x+s w) d w, \\
& u_{\beta}(t, x)=\int_{0}^{t} s^{h} t^{-h} U_{\beta}(s, x) d_{*}, \\
& f_{\infty}(x)=\int \omega_{l}(x,-z) f(x+a z) d z, \\
& f_{\infty}^{(\alpha)}(x)=D_{x}^{\alpha} f_{\infty}(x)=\sum_{\beta}\left(\begin{array}{l}
\alpha \\
\beta
\end{array}\right) \int \omega_{l}^{(\alpha-\beta, \beta)}(x,-z) f(x+a z) d z, \\
& f_{\beta}(x)=\int_{0}^{a} t^{h} U_{\beta}(t, x) d_{*} t,
\end{aligned}
$$

Then

$$
f(x)=F_{1}(x)+F_{2}(x)+F_{3}(x)+F_{4}(x),
$$

where

$$
\left\{\begin{array}{l}
F_{1}(x)=\sum_{|\alpha|=k} \int_{0}^{a} d_{*} t \int M_{\alpha}(x,-z) u^{\alpha}(t, x+t z) d z, \\
F_{2}(x)=\sum_{|\beta| \leqq h} \int_{0}^{a} d_{*} t \int M^{(0, \beta)}(x,-z) t^{h-|\beta|} u_{\beta}(t, x+t z) d z, \\
F_{3}(x)=\sum_{|\alpha|=k} \int_{0}^{a} d_{*} t \int M_{\alpha}(x,-z) t^{k} f_{\infty}^{(\alpha)}(x+t z) d z, \\
F_{4}(x)=\sum_{|\beta| \leqq h} a^{-|\beta|} \int \omega_{m}^{(0, \beta)}(x,-z) f_{\beta}(x+a z) d z \\
\quad+\int \omega_{m}(x,-z) f_{\infty}(x+a z) d z,
\end{array}\right.
$$

In particular,

$$
\begin{aligned}
& F_{1}(x)=\int_{0}^{a} d_{*} t \int M(x,-z) d z \int_{t}^{a} d_{*} s \int L(x+t z,-w) f(x+t z+s w) d w \\
& F_{2}(x)=\int_{0}^{a} d_{*} t \int M(x,-z) d z \int_{0}^{t} d_{*} s \int L(x+t z,-w) f(x+t z+s w) d w \\
& F_{3}(x)=\int_{0}^{a} d_{*} t \int M(x,-z) f_{\infty}(x+t z) d z
\end{aligned}
$$




$$
F_{4}(x)=\int \omega_{m}(x,-z) f(x+a z) d z
$$

(b) For $g \in \mathscr{D}_{\bar{\Omega}}^{\prime}\left(\mathbb{R}^{n} ; X\right),|\alpha| \leqq k,|\beta|=h$, let us write

$$
\begin{aligned}
& V^{\alpha}(s, x)=\int L^{(0, \alpha)}(x+s w, w) g(x+s w) d w, \\
& v^{\alpha}(t, x)=\int_{t}^{a} t^{h} s^{-|\alpha|} V^{\alpha}(s, x) d_{*} s, \\
& V_{\beta}(s, x)=\int L_{\beta}(x+s w, w) g(x+s w) d u, \\
& v_{\beta}(t, x)=\int_{0}^{t} t^{-h} s^{h} V_{\beta}(s, x) d_{*} s, \\
& g_{\infty}(x)=\int_{l} \omega_{l}(x+a z, z) g(x+a z) d z, \\
& g_{\infty}^{(\alpha)}(x)=(-1)^{|\alpha|} a^{-|\alpha|} \int_{l}{ }^{(0, \alpha)}(x+a z, z) g(x+a z) d z, \\
& g_{\beta}(x)=\int_{0}^{h} t^{h} V_{\beta}(t, x) d_{*} t .
\end{aligned}
$$

Then

$$
g(x)=G_{1}(x)+G_{2}(x)+G_{3}(x)+G_{4}(x),
$$

where

$$
\left\{\begin{array}{l}
G_{1}(x)=\sum_{|\alpha| \leqq h} \int_{0}^{a} d_{*} t \int M_{\alpha}(x+t z, z) v^{\alpha}(t, x+t z) d z, \\
G_{2}(x)=\sum_{|\beta|=h} \int_{0}^{a} d_{*} t \int D_{z}^{\beta}\{M(x+t z, z)\} v_{\beta}(t, x+t z) d z, \\
G_{3}(x)=\sum_{|\alpha| \leqq h} \int_{0}^{a} d_{* t} t M_{\alpha}(x+t z) t^{k}(-1)^{|\alpha|} g_{\infty}^{(\alpha)}(x+t z) d z, \\
G_{4}(x)=\sum_{|\beta|=h} s^{-h} \int D_{z}^{\beta}\left\{\omega_{m}(x+a z, z)\right\} g_{\beta}(x+a z) d z \\
\quad+\int \omega_{m}(x+a z, z) g_{\infty}(x+a z) d z .
\end{array}\right.
$$


In particular,

$$
\begin{aligned}
& G_{1}(x)=\int_{0}^{a} d_{*} t \int M(x+t z, z) d z \int_{t}^{a} d_{*} s \int L(x+t z+s w, w) g(x+t z+s w) d w, \\
& G_{2}(x)=\int_{0}^{a} d_{*} t \int M(x+t z, z) d z \int_{0}^{t} d_{*} s \int L(x+t z+s w, w) g(x+t z+s w) d w, \\
& G_{3}(x)=\int_{0}^{a} d_{*} t \int M(x+t z, z) g_{\infty}(x+t z) d z, \\
& G_{4}(x)=\int_{m}\left(\omega_{m}\left(x+u_{\tilde{*}}, z\right) g(x+a z) d z .\right.
\end{aligned}
$$

First we shall show the following.

\section{Lemma 3.4. Let}

$$
K(t, x, z, y)=\sum_{|\alpha|=k} K_{\alpha}^{(0, \alpha, 0)}(t, x, z, y), \quad K_{\alpha} \in \mathscr{K}_{0}
$$

(a) For $f \in \mathscr{D}^{\prime}(\Omega ; X)$ let $u^{\alpha}, u_{\beta}, f_{\infty}^{(\alpha)}$ be the functions given in Theorem 1 , and let $\tilde{K}_{\beta},|\beta| \leqq m$, be the functions defined by (2.4). Then

$$
\begin{aligned}
& \int K(t, x,-z, x+t z) f(x+t z) d z \\
= & \sum_{|\alpha| \leqq k} \int(-1)^{k-|\alpha|} \tilde{K}_{\alpha}(t, x,-z, x+t z)\left\{u^{\alpha}(t, x+t z)+t^{k} f_{\infty}^{(\alpha)}(x+t z)\right\} d z \\
& +\sum_{|\beta| \leqq h}(-1)^{|\beta|} \int t^{h-|\beta|} D_{z}^{\beta}\{K(t, x,-z, x+t z)\} u_{\beta}(t, x+t z) d z .
\end{aligned}
$$

(b) For $g \in \mathscr{D}_{\bar{\Omega}}^{\prime}\left(\mathbb{R}^{n} ; X\right)$ let $v^{\alpha}, v_{\beta}, g_{\infty}^{(\alpha)}$ be the functions given in Theorem 1 , and let $\hat{K}_{\alpha},|\alpha| \leqq m$ be functions defined by (2.3).

Then

$$
\begin{aligned}
& \int K(t, x+t z, z, x) g(x+t z) d z \\
= & \sum_{|\alpha| \leqq h} \int \hat{K}_{\alpha}(t, x+t z, z, x)\left\{v^{\alpha}(t, x+t z)+(-1)^{k} t^{k} g_{\infty}^{(\alpha)}(x+t z)\right\} d z \\
& +\sum_{|\beta|=h} \int D_{z}^{\beta}\{K(t, x+t z, z, x)\} v_{\beta}(t, x+t z) d z .
\end{aligned}
$$


Proof. From Lemma 3.1 Corollary 1 it follows that

$$
f(x)=\int_{0}^{a} d_{*} s \int L(x,-z) f(x+s z) d z+f_{\infty}(x)=\int_{0}^{a} U(s, x) d_{*} s+f_{\infty}(x) .
$$

Substituting this, we obtain

$$
\int K(t, x,-z, x+t z) f(x+t z) d z=u_{1}(t, x)+u_{2}(t, x)+u_{3}(t, x),
$$

where

$$
\begin{aligned}
& u_{1}(t, x)=\int K(t, x,-z, x+t z) d z \int_{t}^{a} U(s, x+t z) d_{*} s \\
& u_{2}(t, x)=\int K(t, x,-z, x+t z) d z \int_{0}^{t} U(s, x+t z) d_{*} s \\
& u_{3}(t, x)=\int K(t, x,-z, x+t z) f_{\infty}(x+t z) d z
\end{aligned}
$$

By Lemma 2.1 Corollary 2 we have

$$
\begin{aligned}
u_{1}(t, x) & =t^{k} \sum_{|\alpha|=k} \sum_{\beta}\left(\begin{array}{l}
\alpha \\
\beta
\end{array}\right) \int K_{\alpha}^{(0,0, \alpha-\beta)}(t, x,-z, x+t z) d z \int_{t}^{a} U^{(\beta)}(s, x+t z) d_{*} s, \\
& =t^{k} \sum_{|\beta| \leqq k} \int(-1)^{k-|\beta|} \tilde{K}_{\beta}(t, x,-z, x+t z) d z \int_{t}^{a} U^{(\beta)}(s, x+t z) d_{*} s
\end{aligned}
$$

Combining this with

$$
\begin{aligned}
U^{(\beta)}(s, x) & =\sum_{\gamma} s^{-|\gamma|}\left(\begin{array}{c}
\beta \\
\gamma
\end{array}\right) \int L^{(\beta-\gamma, \gamma)}(x,-w) f(x+s w) d w \\
& =s^{-k} U^{\beta}(s, x)
\end{aligned}
$$

which is a consequence of Lemma 2.1 Corollary 2, we obtain

$$
u_{1}(t, x)=\sum_{|\alpha| \leqq k} \int(-1)^{k-|\alpha|} \tilde{K}_{\alpha}(t, x,-z, x+t z) u^{\alpha}(t, x+t z) d z
$$

At the same time by Lemma 2.1 Corollary 3 we obtain

$$
\begin{aligned}
\sum_{|\beta| \leqq h} t^{h} D_{x}^{\beta} u_{\beta}(t, x) & =\int_{0}^{t} s^{h} d_{*} s \sum_{|\beta| \leqq h} D_{x}^{\beta} \int L_{\beta}(x,-w) f(x+s w) d w \\
& =\int_{0}^{t} U(s, x) d_{*} s
\end{aligned}
$$


so that, integrating by parts,

$$
\begin{aligned}
& \sum_{|\beta| \leqq h} \int(-1)^{|\beta|} D_{z}^{\beta}\{K(t, x,-z, x+t z)\} t^{h-|\beta|} u_{\beta}(t, x+t z) d z \\
= & \sum_{|\beta| \leqq h} \int K(t, x,-z, x+t z) t^{h} D_{x}^{\beta} u_{\beta}(t, x+t z) d z, \\
= & \int K(t, x,-z, x+t z) d z \int_{0}^{t} U(s, x+t z) d_{*} s, \\
= & u_{2}(t, x) .
\end{aligned}
$$

Also by Lemma 2.1 Corollary 2 we obtain

$$
\begin{aligned}
u_{3}(t, x) & =\sum_{|\alpha|=k} \int K_{\alpha}^{(0, a, 0)}(t, x,-z, x+\ell z) f_{\infty}(x+t z) d z, \\
& =\sum_{|\beta| \leqq k}(-1)^{k-|\beta|} \int \tilde{K}_{\beta}(t, x,-z, x+t z) t^{k} f_{\infty}^{(\beta)}(x+t z) d z ;
\end{aligned}
$$

and this completes the proof of part (a). Similarly, we can verify part (b).

Proof of Theorem 1. By Lemma 3.1 Corollary 1 we obtain

$$
f(x)=\int_{0}^{a} d_{*} t \int M(x,-z) f(x+t z) d z+\int \omega_{m}(x,-z) f(x+a z) d z .
$$

Applying Lemma 3.4 to the first term, we find that this term is equal to $F_{1}+F_{2}+F_{3}$ in the theorem. In order to complete the proof of (a) there only remains to obscrve that the second term is equal to $F_{4}(x)$ in the theorem. This fact is proved by substituting the identity

$$
f(x)=\sum_{|\beta| \leqq h} D^{\alpha} f_{\beta}(x)+f_{\infty}(x)
$$

(Lemma 3.1 Corollary 2) and by integrating by parts. Thus, the proof of part (a) is completed. The proof of part (b) is almost identical with that of part (a).

By our proof of the theorem we have

Corollary. (a) For $f \in \mathscr{D}^{\prime}(\Omega ; X)$ set 


$$
U_{m}(t, x)=\int \omega_{m}(x,-z) f(x+t z) d z
$$

Then

$$
U_{m}(\varepsilon, x)=F_{1, \varepsilon}(x)+F_{2, \varepsilon}(x)+F_{3, \varepsilon}(x)+F_{4}(x),
$$

where $F_{j, \varepsilon}(x), j=1,2,3$, are given by (3.6) with modification that the integration relative to $t$ is taken over $\varepsilon \leqq t \leqq a$.

(b) For $g \in \mathscr{D}_{\Omega}^{\prime}\left(\mathbb{I R}^{n} ; ; \mathrm{Y}\right)$ sct

$$
I_{m}(l, r)=\int(1)_{m}(x+t z, z) g(1+t z) d z
$$

Then

$$
V_{m}(\varepsilon, x)=G_{1, \varepsilon}(x)+G_{2, \varepsilon}(x)+G_{3, \varepsilon}(x)+G_{4}(x),
$$

where $G_{j, \varepsilon}(x), j=1,2,3$, are given by $(3,8)$ with the same modification as that of $F_{j, \varepsilon}$.

\section{§4. A Chlnaraterization of $\mathbb{D}$ inferemtialbility of Furmetions and Distrioibutions}

Lemma $\mathbb{4}_{0} \mathbb{1}_{\text {。 Suppose }} \mathbb{K}(t, x, z, y) \in \overline{\mathscr{K}}_{i}$ and $i$ is a non-negative integer. For $f \in \mathscr{D}^{\prime}(\Omega ; X)$ we write

$$
U(t, x)=\int K(t, x,-z, x+t z) f(x+t z) d z
$$

and for $g \in \mathscr{D}_{\bar{\Omega}}^{\prime}\left(\mathbb{R}^{n} ; X\right)$ we write

$$
V(t, x)=\int K(t, x+t z, z, x) g(x+\iota z) d z .
$$

(I) Assume that $\sigma<i$.

(a) If $1 \leqq p, q \leqq \infty$ and if $f \in B_{p, q}^{\sigma}(\Omega ; X)$, then

$$
t^{-\sigma} U(t, x) \in \mathbb{L}_{*}^{q}\left(\mathbb{I} ; \mathbb{L}^{p}(\Omega ; X)\right) .
$$

(b) If $1 \leqq p, q \leqq \infty$ and if $g \in \mathbb{B}_{p, q, \bar{\Omega}}^{\sigma}\left(\mathbb{R}^{n} ; X\right)$ then

$$
t^{-\sigma} V(t, x) \in L_{*}^{q}\left(I ; L^{p}(\Omega ; X)\right) .
$$


(II) Assume that $\sigma+1 \leqq i$, and that $X$ is a Hilbert space.

(a) If $1<p<\infty$ and if $f \in H_{p}^{\sigma}(\Omega ; X)$, then

$$
t^{-\sigma} U(t, x) \in L^{p}\left(\Omega ; L_{*}^{2}(I ; X)\right) \text {. }
$$

(b) If $1<p<\infty$ and if $g \in H_{p, \bar{\Omega}}^{\sigma}\left(\mathbb{F}^{n} ; X\right)$,

then $t^{-\sigma} V(\iota, x) \in L^{p}\left(\Omega ; L_{*}^{2}(I ; X)\right)$.

Pronf. (I) (a). Case (),$\sigma \cdots 1$. From the identity

$$
\Lambda(1, x,-z, 1+t z)-K(1,1,-\Sigma, 1)+K_{1}(1,1, z),
$$

where $\Lambda_{1}(l, r, z)-\int_{0}^{1} \sum_{j=1}^{n-1} z \frac{\partial R}{\partial \frac{\lambda}{y_{j}}}(I, r, \ldots z, 1+l s z) d s$,

we have

$$
\begin{aligned}
U(t, x) & =\int \mathbb{K}(t, x,-z, x) f(x+t z) d z+t \int K_{1}(t, x, z) \int(x+t z) d z \\
& =U_{0}(\iota, x)+\iota U_{1}(t, x)
\end{aligned}
$$

Since $K \in \overline{\mathscr{K}}_{1}$, it follows that

(4.3) $U_{0}(t, x)=\iint \omega_{1}(x,-w) K(t, x,-z, x)\{f(x+t z)-f(x+t w)\} d z d w$, where $\omega_{1}(x, z)$ is the same function as in Lemma 3.1 Corollary 1, and this gives us, with the aid of Jessen's inequality (or generalized Minkowski's inequality),

$$
\begin{aligned}
\left\|U_{0}(t, x)\right\|_{L^{n}(\Omega ; X)} & \leqq C_{1} \int_{b B} \int_{b B} F_{1}(t z-\iota w) d z d w \\
& =C_{1} b^{n} a_{n} \int_{2 b B} F_{1}(t z) d z
\end{aligned}
$$

where

$$
F_{j}(y)=\left\|\sum_{k=0}^{j}\left(\begin{array}{l}
j \\
k
\end{array}\right)(-1)^{k} f(x+k y)\right\|_{L^{p}\left(\Omega_{3, y} ; X\right)}, \Omega_{j, y}=\bigcap_{k=0}^{j}(\Omega-k y) .
$$

Therefore, we have

$$
\| t^{-\alpha}\left(J_{0}(l, x)\left\|_{L_{*}^{q}\left(I, L^{p}(\Omega ; X)\right)} \leqq C\right\| f \|_{B_{p, q}^{\sigma}(\Omega ; X)}(\Omega)\right.
$$


(see the proof of [18] Lemma 2.3). Since

$$
\left\|U_{1}(t, x)\right\|_{L^{p}(\Omega ; X)} \leqq C\|f\|_{L^{p}(\Omega ; X)},
$$

it follows that

$$
\left\|t^{1-\sigma} U_{1}(t, x)\right\|_{L_{*}^{q}\left(I ; L^{p}(\Omega ; X)\right)} \leqq C\|f\|_{L^{p}(\Omega ; X)},
$$

which, combining with the estimate for $U_{0}$, proves the assertion.

Case $\sigma=1$. From Taylor's expansion it follows that

$$
\begin{aligned}
U(t, x)= & \int K(t, x,-z, x) f(x+t z) d z \\
& +t \int K_{1}(t, x,-z, x) f(x+t z) d z \\
& +t^{2} \int K_{2}(t, x, z) f(x+t z) d z, \\
= & U_{0}(t, x)+t U_{1}(t, x)+t^{2} U_{2}(t, x),
\end{aligned}
$$

where

$$
\begin{aligned}
& K_{1}(t, x, z, y)=\sum_{j=1}^{n} z_{j} \frac{\partial K}{\partial y_{j}}(t, x, z, y), \\
& K_{2}(t, x, z)=\sum_{|\alpha|=2} \frac{2}{\alpha !} \int_{0}^{1}(1-s)^{\alpha} z^{\alpha} K^{(0,0, \alpha)}(t, x,-z, x+t s z) d s .
\end{aligned}
$$

It is easy to see that

$$
\left\|t^{-1} \iota^{2} U_{2}(t, x)\right\|_{L_{*}^{q}\left(I ; L^{p}(\Omega ; X)\right)} \leqq C\|f\|_{L^{p}(\Omega ; X)},
$$

Also, since

$$
\begin{aligned}
K_{1}(t, x, z, y) & =\sum_{j=1}^{n} z_{j} \sum_{k=1}^{n} \frac{\partial^{2} K_{k}}{\partial z_{k} \partial y_{j}}(t, x, z, y), \\
& =\sum_{j, k} \frac{\partial}{\partial z_{k}}\left\{z_{j} \frac{\partial K_{k}}{\partial y_{j}}\right\}-\sum_{j} \frac{\partial K_{j}}{\partial y_{j}} \in \overline{\mathscr{K}}_{1},
\end{aligned}
$$

and since $f \in B_{p, q}^{\varepsilon}(\Omega ; X), 0<\varepsilon<1$, ([18] Theorem 1.1), it follows that

$$
\left\|t^{-1} t U_{1}(t, x)\right\|_{L_{*}^{q}\left(I ; L^{p}(\Omega ; X)\right)}
$$




$$
\leqq a^{\varepsilon}\left\|t^{-\varepsilon} U_{1}(t, x)\right\|_{L_{*}^{q}\left(I ; L^{p}(\Omega ; X)\right)} \leqq C_{1}\|f\|_{B_{p, q}^{\epsilon}(\Omega ; X)} \leqq C\|f\|_{B_{p, q}^{1}(\Omega ; X)}
$$

Hence, to prove the assertion it suffices to estimate $U_{0}$. From the identity

$$
\begin{aligned}
U_{0}(t, x) & =\sum_{k=1}^{n} \int\left(-\frac{\partial}{\partial z_{k}}\right) K_{k}(t, x,-z, x) f(x+t z) d z \\
& =\sum_{k} t \int K_{k}(t, x,-z, x) f_{k}(x+t z) d z, \quad\left(f_{k}=D_{k} f\right) \\
& =\sum_{k} t \iint K_{k}(t, x,-z, x) \omega_{1}(x,-w)\left\{f_{k}(x+t z)\right. \\
& =\sum_{k} t \iint K_{k}(t, x,-z, x) \omega_{1}\left(x,-\frac{z+w}{2}\right)\left\{f_{k}(x+t z)\right\} d z d w \\
& =\iint \tilde{K}(t, x, z, w)\left\{f(x+t z)-2 f\left(x+\frac{t z+t w}{2}\right)\right\} d z d w,
\end{aligned}
$$

where

$$
\tilde{K}(t, x, z, w)=\sum_{k}\left(-\frac{\partial}{\partial z_{k}}\right)\left\{K_{k}(t, x,-z, x) \omega_{1}\left(x,-\frac{z+w}{2}\right)\right\}
$$

(the last identity is obtained by integrating by parts), and from $\iint \tilde{K}(t, x$, $z, w) d z=0$, it follows that $U_{0}(t, x)$ is equal to

$$
\iint \tilde{K}(t, x, z, w)\left\{f(x+t z)-2 f\left(x+\frac{t z+t w}{2}\right)+f(x+t w)\right\} d z d w .
$$

Thus we have

$$
\begin{aligned}
\left\|U_{0}(t, x)\right\|_{L^{p}(\Omega ; X)} & \leqq C \int_{3 b B} d x \int_{b B} F_{2}\left(\frac{t z-t w}{2}\right) d z \\
& =C a_{n} b^{n} 6^{n} \int_{2 b B} F_{2}(\iota z) d z .
\end{aligned}
$$

Using this and [18] Lemma 2.3 (ii), we obtain the estimate 


$$
\left\|t^{-1} U_{0}(t, x)\right\|_{L_{*}^{q}\left(I ; L^{p}(\Omega ; X)\right)} \leqq C\|f\|_{B_{p, q}^{1}(\Omega ; X)} .
$$

Case where $\sigma=k+\theta, 0<\theta \leqq 1$, and $k$ is a positive integer. Since $k<i$, there exists a representation

$$
K(t, x, z, y)=\sum_{|c|-k} K_{c x}^{(0, \alpha, 0)}(l, x, z, y)
$$

therefore, by Lemma 2.1 Corollary 2 we obtain

$$
U(t, x)=t^{k} \sum_{|\beta| \leqq k}(-1)^{k-|\beta|} \int \widetilde{K}_{\beta}(\iota, x,-z, x+\iota z) f^{(\beta)}(x+t z) d z .
$$

This, with the aid of the result for the case $0<0 \leqq 1$, which was already proved, gives the assertion.

Case where $\sigma \leqq 0$. By definition there exists a representation

$$
f(x)=\sum_{|\alpha| \leqq m} D^{\alpha} f_{\alpha}, f_{\alpha} \in B_{p, q}^{\theta}(\Omega ; X),
$$

where $\sigma=-m+\theta, 0<\theta \leqq 1, m$ is a positive integer. Since

$$
U(t, x)=\sum_{|\beta| \leqq m}(-1)^{-|\beta|} \int D_{z}^{\beta}\{K(t, x,-z, x+t z)\} f_{\beta}(x+t z) d z,
$$

the assertion for this case follows from that for the case $0<\sigma \leqq 1$.

(II) (a). First consider the case where $\sigma$ is a non-negative integer $k$. Since

$$
\mathbb{K}(t, x, z, y)=\sum_{|\alpha|=k} \mathbb{K}_{\alpha}^{(0, \alpha, 0)}(t, x, z, y), \quad \mathbb{K}_{\alpha} \in \overline{\mathscr{K}}_{1}
$$

we obtain

$$
t^{-\sigma} U(t, x)=\sum_{|\beta| \leqq k}(-1)^{k-|\beta|} \int \widetilde{K}_{\beta}(t, x,-z, x+t z) f^{(\beta)}(x+t z) d z
$$

Hence, by [21] Theorem 3 Corollary we obtain our assertion.

Next, consider the case where $\sigma$ is a negative integer $-m$. By definition we have

$$
f(x)=\sum_{|\alpha| \leqq m} \mathbb{D}^{\alpha} f_{\alpha}, \quad f_{\alpha} \in \mathbb{L}^{p}(\mathbb{S} ; X)
$$

Hence, it follows that 


$$
t^{-\sigma} U(t, x)=\sum_{|\beta| \leqq m} t^{m-|\beta|} \int D_{z}^{\beta}\{K(t, x,-z, x+t z)\} f_{\beta}(x+t z) d z,
$$

which, with the help of [21] Theorem 3 Corollary, gives us the estimate. Finally, consider the case where $\sigma$ is fractional. Take $m$ such that $m<\sigma<m+1$. Since $m+2 \leqq i$, the results just proved imply that the operator $f \rightarrow U$ is a bounded linear operator from $H_{p}^{m}(\Omega ; X)$ into $L^{p}(\Omega$; $L_{\mathfrak{*}^{\prime}}^{2}{ }^{m}\left(I ; K^{\prime}\right)$ ), and also from $H_{p}^{m+1}(\Omega ; X)$ into $L^{p}\left(\Omega ; L_{*}^{2}{ }^{\prime \prime \prime \prime 1}(I ; Y)\right)$, where

$$
L_{*}^{q, 0}(1 ; X)=L^{q}\left(1, t^{-4 v} 1 d t ; X\right)
$$

Therefore, from the interpolation of operators $([8],[15])$ it follows that this operator is bounded from $H_{p}^{\sigma}(\Omega)$ into $L^{p}\left(\Omega ; L_{*}^{2, \sigma}(I ; X)\right)$, which is our assertion (note that $\left[L^{p}\left(\Omega ; L_{*^{2}}^{2, m}\right), L^{p}\left(\Omega ; L_{*}^{2,{ }^{m+1}}\right)\right]_{\theta}=L^{p}\left(\Omega ; L_{*}^{2, \sigma}\right)$ ).

(I) (b), (II) (b). Since the support of $V(t, x)$ is contained in $\Omega$, we may consider only the case where $\Omega=\mathbb{R}^{n}$, and for that case part (b) is identical with part (a).

Thus the proof of the lemma is complete.

Lemma $\mathbb{H}_{2} \%$ Let $K(t, x, z, y) \in \mathscr{K}_{j}$, where $j$ is a non-negative integer.

(I) Assume that $-\sigma<j, 1 \leqq p, q \leqq \infty$, and that $u \in L_{\text {* }}^{q}\left(I ; L^{p}(\Omega ; X)\right)$.

Then

(a) $\quad\left\|\int_{0}^{a} t^{\sigma} d_{*} t \int \mathbb{K}(t, x,-z, x+t z) u(t, \lambda+t z) d z\right\|_{B p, q(\Omega ; X)}$

$$
\leqq C\|u\|_{L_{*}^{q}\left(I ; L^{p}(\Omega ; X)\right)}
$$

(b) $\quad\left\|\int_{0}^{a} t^{\sigma} d_{*} t \int K(t, x+t z, z, x) u(t, x+t z) d z\right\|_{B p, q\left(\mathbb{R}^{n} ; X\right)}$

$$
\leqq C\|u\|_{L_{*}^{q}\left(I ; L^{p}(\Omega ; X)\right)}
$$

(II) Assume that $j>1-\sigma, 1<p<\infty$, that $X$ is a Hilbert space, and that $u \in L^{p}\left(\Omega ; L_{*}^{2}(I ; X)\right)$.

Then

(a) $\quad\left\|\int_{0}^{a} t^{\sigma} d * t \int K(t, x,-z, x+t z) u(t, x+\iota, z) d z\right\|_{H^{\sigma}(\Omega ; X)}$

$$
\leqq C\left\|_{i} u\right\|_{L^{p}\left(\Omega ; L_{*}^{2}(I ; X)\right)}
$$


(b) $\quad\left\|\int_{0}^{a} t^{\sigma} d_{*} t \int K(t, x+t z, z, x) u(t, x+t z) d z\right\|_{H p\left(\mathbb{R}^{n} ; X\right)}$

$$
\leqq C\|u\|_{L^{p}\left(\Omega ; L_{*}^{2}(I ; X)\right)}
$$

Proof. (I) (a). Case $\sigma>0$. The proof is similar to that of [18] Lemma 4.2 (A) (ii). Case $\sigma \leqq 0$. Write $\sigma=-m+\theta$, where $0<\theta \leqq 1, m$ is a positive integer. Since $j \geqq m$,

$$
K(t, x, z, y)=\sum_{|\alpha|=m} K_{\alpha}^{(0, \alpha, 0)}(t, x, z, y),
$$

so that, using Lemma 2.1 Corollary 3,

$$
\begin{aligned}
& \int_{0}^{a} t^{\sigma} d_{*} t \int K(t, x,-z, x+t z) u(t, x+t z) d z \\
= & \sum_{|\beta| \leqq m} D_{x}^{\beta} \int_{0}^{a} t^{\sigma+m} d_{*} t \int \widehat{K}_{\beta}(t, x,-z, x+t z) u(t, x+t z) d z .
\end{aligned}
$$

From this and from the result for $\sigma>0$ the desired result follows.

(II) (a). Case where $\sigma=k$, a non-negative integer:

For any $|\alpha| \leqq k$, we have

$$
\begin{aligned}
& D_{x}^{\alpha} \int_{0}^{a} t^{k} d_{*} t \int K(t, x,-z, x+t z) u(t, x+t z) d z \\
= & \sum_{\beta}\left(\begin{array}{c}
\alpha \\
\beta
\end{array}\right) \int_{0}^{a} t^{k-|\beta|} d_{*} t \int K^{(\alpha-\beta, \beta, 0)}(t, x,-z, x+t z) u(t, x+t z) d z .
\end{aligned}
$$

Hence, by $[21]$ Theorem 3 Corollary we obtain the estimate.

Case where $\sigma=-m$; a negative integer. Since $1+m \leqq j$, it follows that

$$
K(t, x, z, y)=\sum_{|\alpha|=m} K_{\alpha}^{(0, \alpha, 0)}(t, x, z, y)
$$

with $K_{\alpha} \in \mathscr{K}_{j-m}$. From (4.4) and from [21] Theorem 3 Corollary our assertion follows. For the case where $\sigma$ is fractional the estimate is proved in virtue of the interpolation of operators.

(I) (b), (II) (b). The proof of part (b) is very easy, in view of the result of part (a). (See Proof of Lemma 4.1).

Now, we shall give a characterization of differentiability, which is one of the basic theorems of our investigation: 
rTheorem 2. (Characterization).

(I) Let $1 \leqq p, q \leqq \infty, \sigma$ be a real number, and let $i$ be a non-negative integer with $i>\sigma$.

(a) $f \in B_{p, q}^{\sigma}(\Omega ; X)$ if and only if $f \in \mathbb{W}_{p}^{-\infty}(\Omega ; X)$ and for any $K(x, z) \in \overline{\mathscr{K}}_{i}$

$$
t^{-\sigma} \int K(x,-z) f(x+t z) d z \in L_{*}^{q}\left(\mathbb{I} ; L^{p}(\Omega ; X)\right)
$$

(b) $g \in B_{p, q, \bar{\Omega}}^{\sigma}\left(\mathbb{R}^{n} ; X\right)$ if and only if $g \in W_{p, \bar{\Omega}}^{-\infty}\left(\mathbb{R}^{n} ; X\right)$ and for any $K(x, z) \in \overline{\mathscr{K}}_{i}$,

$$
t^{-\sigma} \int K(x+\iota z, z) g(x+t z) d z \in L_{*}^{q}\left(I ; L^{p}(\Omega ; X)\right) .
$$

(II) Let $1<p<\infty, \sigma$ be a real number and let $i$ be a non-negative integer with $1+\sigma \leqq i$. Assume that $X$ is a Hilbert space. Then

(a) $f \in H_{p}^{\sigma}(\Omega ; X)$ if and only if $f \in W_{p}^{-\infty}(\Omega ; X)$ and for any $K(x, z) \in \overline{\mathscr{K}}_{i}$,

$$
t^{-\sigma} \int K(x,-z) f(x+t z) d z \in L^{p}\left(\Omega ; L_{*}^{2}(I ; X)\right) ;
$$

(b) $g \in H_{p, \bar{\Omega}}^{\sigma}\left(\mathbb{R}^{n} ; X\right)$ if and only if $g \in W_{\bar{p}, \bar{\Omega}}^{-\infty}\left(\mathbb{R}^{n} ; X\right)$ and for any $K(x, z) \in \overline{\mathscr{K}}_{i}$

$$
t^{-\sigma} \int K(x+t z, z) g(x+t z) d z \in L^{p}\left(\Omega ; L_{*}^{2}(I ; X)\right) .
$$

Proof. Necessity: by Lemma 4.1.

Sufficiency: by Lemma 4.2, Lemma 3.2, and by Theorem 1. (choose $m, k$, $l, h$ so that $m-k \geqq j, l \geqq i, k-\sigma>0, h+\sigma>0$, where $j$ is the smallest non-negative integer such that $-\sigma<j$ in (I), or $1-\sigma \leqq j$ in (II).). Since $f \in W_{p}^{-\infty}(\Omega ; X)$, it follows that $f_{\infty}(x) \in W_{p}^{\infty}(\Omega ; X)$. This completes the proof.

Remark. Let $k, h, l, m$ be as in the proof of Theorem 2, and let $U^{\alpha}, U_{\beta}, f_{\beta}, f_{\infty}$ be the functions defined in Theorem 1 . Then $f \in B_{p, q}^{\sigma}(\Omega ; X)$ if and only if

$$
\begin{aligned}
& t^{-\sigma} U^{\alpha}(t, x), t^{-\sigma} U_{\beta}(t, x) \in \mathbb{L}_{*}^{q}\left(I ; L^{p}(\Omega ; X)\right) \text { for }|\alpha|=k,|\beta| \leqq h, \\
& f_{\beta}(x) \in L^{p}(\Omega ; X) \text { for }|\beta| \leqq h
\end{aligned}
$$


and $f_{\infty}(x) \in \mathbb{W}_{p}^{k}(\Omega ; X)$. And $B_{p, q}^{\sigma}(\Omega ; X)$-norm is equivalent with the norm

$$
\begin{aligned}
& \sum_{|\alpha|=k}\left\|t^{-\sigma} U^{\alpha}(t, x)\right\|_{L_{*}^{q}\left(I ; L^{p}(\Omega ; X)\right)}+\sum_{|\beta| \leqq h}\left\|t^{-\sigma} U_{\beta}(t, x)\right\|_{L_{*}^{q}\left(I ; L^{p}(\Omega ; X)\right)} \\
& +\sum_{|\beta| \leqq h}\left\|f_{\beta}(x)\right\|_{L^{\mu}(\Omega ; X)}+\left\|f_{\infty}(x)\right\|_{W_{p}^{k}(\Omega ; X)} .
\end{aligned}
$$

The analogous facts hold for $H_{p}^{\sigma}, B_{p, q, \bar{\Omega}}^{\sigma}$ or $H_{p, \Omega}^{\sigma}$.

Cormllmiry 1. Under the same assumptions as in the theorem, (I) (a) $f \in B_{p, q}^{\sigma}(\Omega ; X)$ if and only if $f \in W_{p}^{-\infty}(\Omega ; X)$ and for any $K(x, z) \in \mathscr{K}_{0}$ and for any $|\alpha| \geqq i$

$$
t^{|\alpha|-\sigma} D_{x}^{\alpha} \int K(x,-z) f(x+t z) d z \in L_{*}^{q}\left(I ; L^{p}(\Omega ; X)\right)
$$

(b) $g \in B_{p, q, \bar{\Omega}}^{\sigma}\left(\mathbb{R}^{n} ; X\right)$ if and only if $g \in W_{p, \bar{\Omega}}^{-\infty}\left(\mathbb{R}^{n} ; X\right)$ and for any $K(x, z) \in \mathscr{K}_{0}$ and for any $|\alpha| \geqq i$

$$
t^{|\alpha|-\sigma} D_{x}^{\alpha} \int \mathbb{K}(x+t z, z) g(x+t z) d z \in L_{*}^{q}\left(I ; L^{p}(\Omega ; X)\right)
$$

(II) (a) $f \in H_{p}^{\sigma}(\Omega ; X)$ if and only if $f \in W_{p}^{-\infty}(\Omega ; X)$ and for any $K(x, z) \in \mathscr{K}_{0}$ and any $|\alpha| \geqq i$

$$
t^{|a|-\sigma} D_{x}^{a} \int K(x,-z) f(x+t z) d z \in L^{p}\left(\Omega ; L_{*}^{2}(I ; X)\right)
$$

(b) $g \in H_{p, \bar{\Omega}}^{\sigma}\left(\mathbb{R}^{n} ; X\right)$ if and only if $f \in W_{p, \bar{\Omega}}^{-\infty}\left(\mathbb{R}^{n} ; X\right)$ and for any $K(x, z) \in \mathscr{K}_{0}$ and for any $|\alpha| \geqq i$

$$
t^{|\alpha|-\sigma} D_{x}^{\alpha} \int K(x+t z, z) g(x+t z) d z \in L^{p}\left(\Omega ; L_{*}^{2}(I ; X) .\right.
$$

Proof. (I) (a), (II) (a). From Lemma 2.1 Corollary 2 it follows that

$$
\begin{aligned}
D_{x}^{\alpha} \int K(x,-z) f(x+t z) d z & =\sum_{\beta}\left(\begin{array}{l}
\alpha \\
\beta
\end{array}\right) t^{-|\beta|} \int K^{(\alpha-\beta, \beta)}(x,-z) f(x+t z) d z \\
& =\sum_{\beta}\left(\begin{array}{l}
\alpha \\
\beta
\end{array}\right) t^{-|\beta|} U_{\alpha, \beta}(t, x) .
\end{aligned}
$$

On the other hand if $f \in B_{p, q}^{\sigma}(\Omega ; X)$ (or $\in H_{p}^{\sigma}(\Omega ; X)$ ), then $f \in B_{p, q}^{\sigma-|\alpha|+|\beta|}$ (or $\in H_{p}^{\sigma-|\alpha|+|\beta|}$ ), but $K^{(\alpha-\beta, \beta)} \in \overline{\mathscr{K}}_{|\beta|},|\beta|>\sigma-|\alpha|+|\beta|$ when $|\alpha| \geqq i$. 
Therefore, by the theorem we obtain

$$
t^{|\alpha|-|\beta|} \sigma U_{\alpha, \beta}(t, x) \in L_{*}^{q}\left(I ; L^{p}(\Omega ; X)\right)\left(\text { or } \in L^{p}\left(\Omega ; L_{*}^{2}(I ; X)\right)\right),
$$

which gives

$$
f^{|\alpha|} \quad \sigma \mid J_{x}^{a} \int K(x,-z) f(x+t z) d z \in L_{*}^{q}\left(I ; L^{p}(\Omega ; \lambda)\right)\left(\text { or } \in L^{p}\left(\Omega ; L_{*}^{2}(I ; X)\right)\right) \text {. }
$$

Conversely, assume that this holds for any $K \in \mathscr{K}_{0}$ and $|\alpha| \geqq i$.

Let $K(x, z)$ be

$$
K(x, z)=\sum_{|\alpha|=i} K_{\alpha}^{(0, \alpha)}(x, z)
$$

Then, we obtain by Lemma 2.1 Corollary 3 that

$$
\begin{array}{r}
t^{-\sigma} \int K(x,-z) f(x+t z) d z=t^{i-\sigma} \sum_{|\beta| \leqq i} D_{x}^{\beta} \int \hat{K}_{\beta}(x,-z) f(x+t z) d z \\
\in L_{*}^{q}\left(I ; L^{p}(\Omega ; X)\right)\left(\text { or } \in L^{p}\left(\Omega ; L_{*}^{2}(I ; X)\right)\right) .
\end{array}
$$

(I) (b), (II) (b). Using the identities given in Lemma 2.1 Corollary 2 and in its Corollary 3 , we can verify part (b).

Corollimin 2. Let $m$ be a non-negative integer.

(I) Assume that $1 \leqq p, q \leqq \infty$. Then $f \in B_{p, q}^{\sigma}(\Omega ; X)$ if and only if $f \in W_{p}^{-\infty}(\Omega ; X)$ and $D^{a} f \in B_{p, q}^{\sigma-m}(\Omega ; X)$ for any $|\alpha|=m$;

(II) Assume that $X$ is a Hilbert space and that $1<p<\infty$. Then, $f \in H_{p}^{\sigma}(\Omega ; X)$ if and only if $f \in W_{p}^{-\infty}(\Omega ; X)$ and $D^{\alpha} f \in H_{p}^{\sigma-m}(\Omega ; X)$ for any $|\alpha|=m$;

Proof. (I) It suffices to prove "if" part. Let $i$ be a non-negative integer such that $i>\sigma, m$, and let

$$
K(x, z)=\sum_{|\alpha|=m} K_{\alpha}^{(0, \alpha)}(x, z), K_{\alpha} \in \overline{\mathscr{K}}_{i-m}
$$

Then, using the identity

$$
t^{-\sigma} \int K(x,-z) f(x+t z) d z=\sum_{\mid \alpha !=m} t^{m-\sigma} \int \mathbb{K}_{\alpha}(x,-z) f^{(\alpha)}(x+t z) d z
$$

and the theorem, we obtain the assertion. 
Corøllary 3. If $\sigma<\tau$ then $B_{p, q}^{\sigma}(\Omega ; X) \supset B_{p, q}^{\tau}(\Omega ; X)$.

\section{$\S 5$. Fractional Derivatives}

Let $f \in \mathscr{D}^{\prime}(\Omega ; X), g \in \mathscr{D}_{\bar{\Omega}}^{\prime}\left(\mathbb{R}^{n} ; X\right)$, and let $\lambda$ be a complex number. Then by Lemma 2.2 Corollary 1 and by Example 2.1 the integrals

$$
F_{\lambda}(x)=\int_{0}^{a} t^{\lambda} d_{*} t K(t, x,-z, x+t z) f(x+t z) d z
$$

and

$$
G_{\lambda}(x)=\int_{0}^{a} t^{\lambda} d_{*} t \int K(t, x+t z, z, x) g(x+t z) d z
$$

exist when $K(t, x, z, y) \in \mathscr{K}_{i}, i+\operatorname{Re} \lambda>0$.

In this section we shall prove a theorem which shows us that $F_{\lambda}$ and $G_{\lambda}$ are, in a sense, generalization of the fractional derivative and the fractional integral.

Theorem 3. Let $i, j$ be non-negative integers and let $K(t, x, z, y)$ $\in \mathscr{K}_{i}$.

(I) Assume that $j>\sigma, \sigma+\operatorname{Re} \lambda>j-i$ and $1 \leqq p, q \leqq \infty$. Then,

(a) the operator $f \rightarrow F_{\lambda}$ is a bounded linear operator from $B_{p, q}^{\sigma}(\Omega ; X)$ into $B_{p, q}^{\sigma+R e \lambda}(\Omega ; X)$;

(b) the operator $g \rightarrow G_{\lambda}$ is a bounded linear operator from $B_{p, q, \bar{\Omega}}^{\sigma}\left(\mathbb{R}^{n} ; X\right)$ into $B_{p, q, \bar{\Omega}}^{\sigma+R} \lambda\left(\mathbb{R}^{n} X\right)$.

(II) Assume that $j>\sigma, \sigma+\operatorname{Re} \lambda>j-i+1, X$ is a Hilbert space, and that $1<p<\infty$. Then, (a) the operator $f \rightarrow F_{\lambda}$ is a bounded linear operator from $H_{p}^{\sigma}(\Omega ; X)$ into $H_{p}^{\sigma+R e \lambda}(\Omega ; X)$; (b) the operator $g \rightarrow G_{\lambda}$ is a bounded linear operator from $H_{p, \bar{\Omega}}^{\sigma}\left(\mathbb{R}^{n} ; X\right)$ into $H_{p, \bar{\Omega}}^{\sigma+R e \lambda}\left(\mathbb{R}^{n} ; X\right)$.

Proof. We shall prove only part (I) (a). The other parts are proved similarly.

(i) Case $j \leqq i$. From Lemma 3.4 it follows that 


$$
\begin{aligned}
& \int K(t, x,-z, x+t z) f(x+t z) d z \\
= & \sum_{|\alpha| \leqq k} \int(-1)^{k-|\alpha|} \tilde{K}_{\alpha}(t, x,-z, x+t z)\left\{u^{\alpha}(t, x+t z)+t^{k} f_{\infty}^{(\alpha)}(x+t z)\right\} d z \\
& +\sum_{|\beta| \leqq h}(-1)^{|\beta|} \int t^{h-|\beta|} D_{z}^{\beta}\{K(t, x,-z, x+t z)\} u_{\beta}(t, x+t z) d z,
\end{aligned}
$$

where $K(t, x, z, y)=\Sigma K_{\alpha}^{(0, \alpha, 0)}(t, x, z, y)$, and $u^{\alpha}, u_{\beta}, f_{\infty}$ are the functions defined in Theorem 1 with $h+\sigma>0, l-h>\sigma, k>\sigma, i-k>-\sigma-\operatorname{Re} \lambda$.

With the aid of Theorem 2 and Lemma 4.2 this shows our assertion.

(ii) Case $j>i$. Let $|\alpha|=j-i$. Then, from Lemma 2.1 Corollary 2 it follows that

$$
D^{\kappa} F_{\lambda}(x)=\sum_{\beta}\left(\begin{array}{l}
\alpha \\
\beta
\end{array}\right) \int_{0}^{a} t^{\lambda-|\beta|} d_{*} t \int K^{(\alpha-\beta, \beta, 0)}(t, x,-z, x+t z) f(x+t z) d z
$$

Since $B_{p, q}^{\sigma} \subset B_{p, q}^{\sigma-j+i+|\beta|}(|\beta| \leqq j-i)$, and since $K^{(\alpha-\beta, \beta, 0)} \in K_{i+|\beta|}$, with the aid of the result in (i), this gives $D^{\alpha} F_{\lambda}(x) \in B_{p, q}^{\sigma-j+R e \lambda}$. Similarly, we have $F_{\lambda} \in B_{p, q}^{\sigma-j+i+\mathrm{Re} \lambda}$, and by Theorem 2 Corollary 2 we obtain $F_{\lambda} \in B_{p, q}^{\sigma+\mathrm{Re}}(\Omega ; X)$. This completes the proof.

Corollary $\mathbf{1}$. Let $m$ be a positive integer.

(I) Assume that $1 \leqq p, q \leqq \infty$. Then,

(a) for any $f \in B_{p, q}^{\sigma}(\Omega ; X)$ there exist $f_{\alpha} \in B_{p, q}^{m+\sigma}(\Omega ; X),|\alpha| \leqq m$, such that $f=\sum_{|\alpha| \leqq m} D^{\alpha} f$

(b) for any $g \in B_{p, q, \bar{\Omega}}^{\sigma}\left(\mathbb{R}^{n} ; X\right)$ there exist $g_{\alpha} \in B_{p, q, \bar{\Omega}}^{m+\sigma}\left(\mathbb{R}^{n} ; X\right),|\alpha| \leqq m$, such that $g=\sum_{|\alpha| \leqq m} D^{\alpha} f_{\alpha}$.

(II) Assume that $X$ is a Hilbert space and that $1<p<\infty$. Then, (a) for any $f \in H_{p}^{\sigma}(\Omega ; X)$ there exist $f_{\alpha} \in H_{p}^{m+\sigma}(\Omega ; X),|\alpha| \leqq m$, such that $f=\sum_{|\alpha| \leqq m} D^{\alpha} f_{\alpha} ;(b)$ for any $g \in H_{p, \bar{\Omega}}^{\sigma}\left(\mathbb{R}^{n} ; X\right)$ there exist $g_{\alpha} \in H_{p, \bar{\Omega}}^{m+\sigma}\left(\mathbb{R}^{n} ; X\right)$, $|\alpha| \leqq m$, such that $g=\sum_{|\alpha| \leqq m} D^{\alpha} g_{\alpha}$.

Proof. These are direct consequences of the theorem and Lemma 3.1 Corollary 2.

\section{Cørollary 2。}

$$
\bigcup_{-\infty<\sigma<\infty} B_{p, q}^{\sigma}(\Omega ; X)=W_{p}^{-\infty}(\Omega ; X) .
$$


If $p \leqq q$ then $W_{p}^{-\infty}(\Omega ; X) \subset W_{q}^{-\infty}(\Omega ; X)$.

Proof. By Corollary 1 and by $[20]$ Theorem 1 (this theorem is also valid for $X$-valued functions, cf. $\S 7$ ).

Example. Let $0<\operatorname{Re} \lambda<1, m \neq 0, x \in \mathbb{R}^{1}$, and $\varphi \in C_{0}^{\infty}\left(\mathbb{R}^{1}\right)$ with support contained in $-2<z<-1$. Then

$$
\begin{aligned}
& \int_{0}^{1} t^{\lambda} d_{*} t \varphi(-z) e^{i m(x+t z)} d z \\
= & e^{i m x} \int_{0}^{\infty} t^{\lambda} d_{*} t \int_{-\infty}^{\infty} e^{i m t z} \varphi(z) d z+c_{\infty}(\lambda, m) e^{t m x}
\end{aligned}
$$

where

$$
c_{\infty}(\lambda, m)=\int_{1}^{\infty} t^{\lambda} d_{*} t \int_{\mathbb{R}} e^{i m t z} \varphi(-z) d z .
$$

A simple calculation shows that

$$
\int_{0}^{\infty} t^{\lambda} d_{*} t \int_{-\infty}^{\infty} e^{-i m t z} \varphi(z) d z \begin{cases}=m^{\lambda} \int_{0}^{\infty} \hat{\varphi}(t) t^{\lambda-1} d t & \text { for } m>0, \\ =-m^{\lambda} \int_{-\infty}^{0} \hat{\varphi}(t) t^{\lambda-1} d t & \text { for } m<0,\end{cases}
$$

where

$$
\hat{\varphi}(t)=\int_{-\infty}^{\infty} \varphi(z) e^{-i t z} d z .
$$

Since $\hat{\varphi}(t)$ is an entire function such that $|\hat{\varphi}(t)| \leqq C e^{-\operatorname{Im} t}$ for $\operatorname{Im} t \geqq 0$, we obtain

$$
\int_{0}^{\infty} \hat{\varphi}(t) t^{\lambda-1} d t=-\int_{-\infty}^{0} \hat{\varphi}(t) t^{\lambda-1} d t=c(\lambda) i^{\lambda} .
$$

Therefore, for $f(x)=\sum_{-\infty}^{\infty} c_{m} e^{i m x}$ we have

$$
\int_{0}^{1} t^{\lambda} d * t \int \varphi(-z) f(x+t z) d z=c(\lambda) \sum_{-\infty}^{\infty}(i m)^{\lambda} c_{m} e^{i m x}+f_{\infty}(x),
$$

where 


$$
f_{\infty}(x)=\sum_{-\infty}^{\infty} c_{\infty}(\lambda, m) c_{m} e^{i m x} \in C^{\infty}\left(\mathbb{R}^{1}\right),
$$

since

$$
\left|c_{\infty}(\lambda, m)\right| \leqq C_{\lambda, k}|m|^{-k}
$$

for any $k>0$. Note that

$$
\sum_{-\infty}^{\infty}(i m)^{\lambda} c_{m} c^{i m x}
$$

is the fractional derivative in the sense of Harly-Littlewood [11].

Next, we shall discuss the product of the operators defined by (5.1) or $(5.2)$.

Theorem 4. Let $i$ and $j$ be non-negative integers, $\mathbb{K}(t, x, z, y) \in \mathscr{K}_{i}$, $L(t, x, z, y) \in \mathscr{K}_{j}$, and let $\lambda$ and $\mu$ be numbers with $i+\operatorname{Re} \lambda>0, j+\operatorname{Re} \mu>0$. Then there exist $M_{k}(t, x, z, y) \in \mathscr{K}_{i+j-k}, k=0, \ldots, i$, and $\tilde{M}(t, x, z, y) \in \mathscr{K}_{0}$ such that

$$
\begin{gathered}
\int_{0}^{a} s^{\lambda} d_{*} \int K(s, x,-w, x+s w) d w \int_{0}^{a} t^{\mu} d{ }_{*} t \\
\int L(x, x+s w,-z, x+s w+t z) f(x+s w+t z) d z \\
=\sum_{k=0}^{i} \int_{0}^{a} t^{\lambda+\mu+k} d{ }_{*} t \int M_{k}(t, x,-z, x+t z) f(x+t z) d z \\
+\int_{a}^{2 a} d_{*} \int \tilde{M}(t, x,-z, x+t z) f(x+t z) d z
\end{gathered}
$$

holds for any $f \in \mathscr{D}^{\prime}(\Omega ; X)$; and there exist $N_{k}(t, x, z, y) \in \mathscr{K}_{j+i-k}, k=0, \ldots$, $i$, and $\tilde{N}(t, x, z, y) \in \mathscr{K}_{0}$ such that for $g \in \mathscr{D}_{\bar{\Omega}}^{\prime}\left(\mathbb{R}^{n} ; X\right)$

$$
\begin{aligned}
\int_{0}^{a} s^{\lambda} d_{*} s \int K(s, x+s w, w, x) d w \int_{0}^{a} t^{\mu} d d_{*} i \\
\qquad L(x, x+s w+t z, z, x+s w) g(x+s w+t z) d z \\
=\sum_{k=0}^{i} \int_{0}^{a} t^{\lambda+\mu+k} d_{*} t \int N_{k}(t, x+t z, z, x) g(x+t z) d z \\
+\int_{a}^{2 a} d_{*} \int \tilde{N}(t, x+t z, z, x) g(x+i z) d z .
\end{aligned}
$$


To prove the theorem we shall need the following lemma.

Lemma 5.I. Let $K(t, x, z, y), L(t, x, z, y) \in \mathscr{K}_{0}$, and let $\operatorname{Re} \lambda$, $\operatorname{Re} \mu>0$. Then

$$
\begin{aligned}
& \int_{0}^{\infty} \frac{s^{\mu-n} d_{*} s}{(1+s)^{\lambda+\mu-2 n}} \int K\left(\frac{t}{1+s},\right.x,-(1+s) w, x+t w) \\
& L\left(\frac{s t}{1+s}, x+t w, \frac{1+s}{s}(z+w), y\right) d w
\end{aligned}
$$

belongs to $\mathscr{K}_{0}$.

Proof. Let

$$
\begin{array}{r}
M(t, s, x, z, y)=\int K\left(\frac{t}{1+s}, x,-(1+s) w, x+t w\right) \\
L\left(\frac{s t}{1+s}, x+t w, \frac{1+s}{s}(z+w), y\right) d w .
\end{array}
$$

Then

$$
\begin{aligned}
& |M(t, s, x, z, y)| \leqq C_{1} \int_{\frac{b}{1+s} B} d w=C_{1} a_{n} b^{n}(1+s)^{-n} \\
& |M(t, s, x, z, y)| \leqq C_{1} \int_{\frac{b s}{1+s} B-z} d w=C_{1} a_{n} b^{n}\left(\frac{s}{1+s}\right)^{n}
\end{aligned}
$$

where $a_{n}$ is the volume of the unit ball $B$ in $\mathbb{R}^{n}$, so the integral

$$
\int_{0}^{\infty} M(t, s, x, z, y) \frac{s^{\mu-n}}{(1+s)^{\lambda+\mu-2 n}} d_{*} s=M(t, x, z, y)
$$

is uniformly convergent and a bounded function of $t, x, z, y$. Similarly, for any $\alpha, \beta, \gamma$, the integral

$$
\int_{0}^{\infty} D_{x}^{\alpha} D_{y}^{\beta} D_{z}^{\gamma} M(t, s, x, z, y) \frac{s^{\mu-n}}{(1+s)^{\lambda+\mu-2 n}} d_{*} s
$$

is uniformly convergent and a bounded function of $t, x, z, y$. Therefore, $\mathbb{M}(t, x, z, y)$ is a $\mathscr{B}^{\infty}\left(\mathbb{R}^{3 n}\right)$-valued continuous function of $0 \leqq t<t_{0}$.

Next consider the support of $M(t, x, z, y)$. Let us assume that for some $s M(t, s, x, z, y) \neq 0$. 
Then for some $w$

$$
\begin{aligned}
& -(1+s) w \in-A\left(\frac{t}{1+s}, x\right), \\
& \frac{1+s}{s}(z+w) \in-A\left(\frac{t s}{1+s}, x+t w\right),
\end{aligned}
$$

so that, putting $v=(1+s) w$ and $t_{1}=\frac{t}{1+s}$, we have

$$
v \in A\left(t_{1}, x\right),-t z-t_{1} v \in\left(t-t_{1}\right) A\left(t-t_{1}, x+t_{1} v\right),
$$

and, therefore, $-z \in A(t, x)$. Thus $M \in \mathscr{K}_{0}$.

Proof of Theorcm 4. Let $f \in \mathscr{D}^{\prime}(\Omega ; Y)$, and assume that $K=\sum_{|\alpha|=1} K_{\beta}^{(0, \beta, 0)}, L=\sum_{|\beta|=j} L_{\beta}^{(0, \beta, 0)}$. By Lemma 2.2 we have

$$
\begin{gathered}
\int K(s, x,-w, x+s w) d w \int_{0}^{a} t^{\mu} d * t \\
\int L(t, x+s w,-z, x+s w+t z) f(x+s w+t z) d z \\
=\int_{0}^{a} t^{\mu} U(s, t, x) d_{*} t_{9}
\end{gathered}
$$

where

$$
\begin{aligned}
& U(s, t, x)=\int K(s, x,-w, x+s w) d w \\
& \quad \int L(i, x+s w,-z, x+s w+t z) f(x+s w+t z) d z
\end{aligned}
$$

Since by Lemma 2.1 Corollary 2 we find that

$$
\begin{aligned}
& D_{x}^{\gamma} \int L(t, x,-z, x+t z) f(x+t z) d z \\
= & \sum_{|\beta|=j} t^{j} \sum_{\delta} \sum_{\varepsilon}\left(\begin{array}{l}
\beta \\
\delta
\end{array}\right)\left(\begin{array}{l}
\gamma \\
\varepsilon
\end{array}\right) \int t^{-|\varepsilon|} L_{\beta}^{(\gamma-\varepsilon, \varepsilon, \beta-\delta)}(t, x,-z, x+t z) f^{(\delta)}(x+t z) d z, \\
= & t^{j} \sum_{|\beta|=j} \sum_{\varepsilon} \sum_{\eta}\left(\begin{array}{l}
\varepsilon \\
\eta
\end{array}\right)\left(\begin{array}{c}
\gamma \\
\varepsilon-\beta
\end{array}\right) \int L_{\beta}^{(\gamma+\beta-\varepsilon, 0, \varepsilon-\eta)}(t, x,-z, x+t z) f^{(\eta)}(x+t z) d z,
\end{aligned}
$$


we obtain that $U(s, t, x)$ is equal to

$$
t^{i+j} \sum_{\varepsilon} \sum_{\eta}\left(\begin{array}{l}
\varepsilon \\
\eta
\end{array}\right) \sum_{|\gamma| \leqq i} \sum_{|\beta|=j}\left(\begin{array}{c}
\gamma \\
\varepsilon-\beta
\end{array}\right) U_{\beta \gamma \varepsilon \eta}(s, t, x),
$$

with

$$
\begin{aligned}
& U_{\beta \gamma \varepsilon \eta}(s, l, x)=\sum_{|\alpha|=j}\left(\begin{array}{l}
\alpha \\
\gamma
\end{array}\right) \int K_{\alpha}^{(0,0, \alpha-\beta)}(s, x,-w, x+s w) d w \\
& \times \int L_{\beta}^{\left(\gamma \mid \beta-\varepsilon, 0, \varepsilon-\eta^{\prime}\right.}(t, .++s w,-z, . x+s w+l z) f^{(\eta)}(x+s w+t z) d z .
\end{aligned}
$$

Hence, by the fact that

$$
\begin{aligned}
& \int_{0}^{a} s^{\lambda} d_{*} s \int_{0}^{a} t^{\mu} U(s, t, x) d_{*} t \\
= & \left\{\int_{0}^{a} t^{\lambda \mid \mu} d_{*} t \int_{0}^{\infty} \frac{s^{\mu} d_{*} s}{(1+s)^{\lambda+\mu}}+\int_{a}^{2 a} t^{\lambda ! \mu} d_{*} t \int_{(t-a) / a}^{u /(t-a)} \frac{s^{\mu} d_{*} s}{(1+s)^{\lambda+\mu}}\right\} U\left(\frac{t}{1+s}, \frac{t s}{1+s}, x\right), \\
= & F(x)+F_{0}(x)
\end{aligned}
$$

and by interchanging the variables

$$
\begin{aligned}
& \frac{t}{1+s} w=t \tilde{w}, \frac{t}{1+s} w+\frac{t s}{1+s} z=t \tilde{z}, \\
& d w d z=(1+s)^{2 n} s^{-n} d \tilde{w} d \tilde{z}
\end{aligned}
$$

we have

$$
F(x)=\sum_{i+j \geqq|\varepsilon| \geqq j} \int_{0}^{a} t^{\lambda+\mu+i+j} d_{*} t \int \sum_{\gamma}\left(\begin{array}{l}
\varepsilon \\
\gamma
\end{array}\right) M_{\varepsilon}^{(0,0, \varepsilon-\eta)}(t, x,-z, x+t z) f^{(\eta)}(x+\iota z) d z,
$$

(by Lemma 2.1 Corollary 2)

$$
\begin{aligned}
& =\sum_{k=0}^{i} \int_{0}^{a} t^{\lambda+\mu+k} d d_{*} \int_{|\varepsilon|=i+j-k} M_{\varepsilon}^{(0, \varepsilon, 0)}(t, x,-z, x+t z) f(x+t z) d z, \\
& =\sum_{k=0}^{i} \int_{0}^{a} t^{\lambda+\mu+k} d_{*} t M_{k}(t, x,-z, x+t z) f(x+t z) d z,
\end{aligned}
$$

where 


$$
\begin{aligned}
& M_{\varepsilon}(t, x, z, y) \\
& =\sum_{|a|=i} \sum_{\gamma}\left(\begin{array}{l}
\alpha \\
\gamma
\end{array}\right) \sum_{|\beta|=j}\left(\begin{array}{c}
\gamma \\
\varepsilon-\beta
\end{array}\right) \int_{0}^{\infty} \frac{s^{\mu+j-n} d_{*} S}{(1+s)^{\lambda+\mu+i+j-2 n}} \\
& \times \int K_{\alpha}^{(0,0, a-\gamma)}\left(\frac{t}{1+s}, x,-(1+s) w, x+t w\right) \\
& L_{i j}^{(y+j s, n, 0)}\left(\frac{s l}{1+s}, x+\left(w, \frac{1+s}{s}(w+z), y\right) d w,\right. \\
& M_{k}(t, 1, z, y)=\sum_{|c|-i+1, k} M_{\varepsilon}^{(0, \ldots u)}(t, 1, \therefore, r)
\end{aligned}
$$

The fact that $M_{k} \in \mathscr{K}_{i \mid j-k}$ is given by lemma 5.1. The term $H_{0}(x)$ can be computed similarly. The second part of the theorem can be argued in the same way and the proof of the theorem completes.

\section{§6 Appraximation and Extension}

In this section we shall consider two problems. The first is the approximation problem: Can any element in a given space (Besov or Sobolev) be approximated by an infinitely differentiable function? The second is the extension problem: How can elements in a given space of distributions defined on $\Omega$ be extended to $\mathbb{F}^{\prime \prime}$ " with preservation of their differentiability properties? In the following of this section we shall assume that $X$ is a Banach space, $\sigma$ a real number, $1 \leqq p \leqq \infty, 1 \leqq q \leqq \infty$.

Theorem 5. (Approximation) Let $m$ be a posilive integer such that $m>\sigma+1$. Assume that $\omega(z) \in \mathbb{C}_{0}^{\infty}\left(\mathbb{R}^{n}{ }^{n}\right), \operatorname{supp}(\omega) \subset B$,

$$
\int \omega(z) d z=1
$$

For $f \in \mathscr{D}^{\prime}(\Omega ; X)$ and $g \in \mathscr{D}_{\bar{\Omega}}^{\prime}\left(\mathbb{R}^{n} ; X\right)$ sel

$$
\begin{aligned}
& U_{m}(t, x)=\int \omega_{m}(x,-z) f(x+i z) d z, \\
& V_{m}(t, x)=\int \omega_{m}(x+i, z, z) g(x+i, z) d ;,
\end{aligned}
$$


where

$$
\omega_{m}(x, z)=\sum_{|\alpha|<m} \frac{1}{\alpha !} D_{z}^{\alpha}\left\{z^{\alpha} \omega(-z-\Psi(x))\right\} .
$$

(I) If $f \in B_{p, q}^{\sigma}(\Omega ; X)$, then

$$
U_{m}(t, x) \rightarrow f(x) \quad \text { in } B_{p, q}^{\sigma}(\Omega ; X) \text { as } t \rightarrow 0 .
$$

If $g \in B_{p, q, \bar{\Omega}}^{\sigma}\left(\boldsymbol{R}^{n} ; X\right)$, then $\operatorname{supp} V_{m}(t, x) \subset \Omega$ and

$$
V_{m}(t, x) \rightarrow g(x) \quad \text { in } B_{p, q}^{\sigma}\left(\mathbb{R}^{n} ; X\right) \text { as } t \rightarrow 0 .
$$

(II) Assume moreover that $X$ is a Hilbert space and $1<p<\infty$.

If $f \in H_{p}^{\sigma}(\Omega ; X)$, then

$$
U_{m}(t, x) \rightarrow f(x) \quad \text { in } \quad H_{p}^{\sigma}(\Omega ; X) \text { as } t \rightarrow 0 .
$$

If $g \in H_{p, \bar{\Omega}}^{\sigma}\left(\mathbb{R}^{n} ; X\right)$ then $\operatorname{supp} V_{m}(t, x) \subset \Omega$ and

$$
V_{m}(t, x) \rightarrow g(x) \quad \text { in } \quad H_{p}^{\sigma}\left(\mathbb{R}^{n} ; X\right) \text { as } t \rightarrow 0 .
$$

Proof. From Theorem 1 Corollary it follows that

$$
U_{m}(\varepsilon, x)=F_{1, \varepsilon}(x)+F_{2, \varepsilon}(x)+F_{3, \varepsilon}(x)+F_{4}(x),
$$

(Here we make use of the same notations as in Theorem 1) And from Lemma 4.1 and Lemma 3.3 it follows that, taking $k=m$,

$$
t^{-\sigma} u^{\alpha}(t, x), t^{-\sigma} u_{\beta}(t, x), t^{-\sigma} t^{|\alpha|} f_{\infty}^{(\alpha)}(x) \in L_{*}^{q}\left(I ; L^{p}(\Omega ; X)\right),
$$

Therefore, $F_{1, \varepsilon}, F_{2, \varepsilon}$ and $F_{3, \varepsilon}$ converge in $B_{p, q}^{\sigma}(\Omega)$. Combining this with the fact that

$$
U_{m}(\varepsilon, x) \rightarrow f(x) \quad \text { in } \quad \mathscr{D}^{\prime}(\Omega ; X) \text { as } \varepsilon \rightarrow 0 \text {. }
$$

which follows from Lemma 2.1, we obtain the first part of the theorem. The remainder parts are verified in the same way.

Corollary. (I) $W_{p}^{\infty}(\Omega ; X)$ is dense in $B_{p, q}^{\sigma}(\Omega ; X), W_{p, \bar{\Omega}}^{\infty}\left(\mathbb{R}^{n} ; X\right)$ is dense in $B_{p, q, \bar{\Omega}}^{\sigma}\left(\mathbb{R}^{n} ; X\right)$, also $C_{0}^{\infty}(\Omega ; X)$ is dense in $B_{p, q, \bar{\Omega}}^{\sigma}\left(\mathbb{R}^{n} ; X\right)$ (II) Assume that $X$ is a Hilbert space and $1<p<\infty$. Then, $W_{p}^{\infty}(\Omega ; X)$ 
is dense in $H_{p}^{\sigma}(\Omega ; X) . \quad \mathbb{W}_{p, \bar{\Omega}}^{\infty}(\Omega ; X)$ is dense in $H_{p, \Omega}^{\sigma}\left(\mathbb{P}^{n} ; X\right)$, also $C_{0}^{\infty}(\Omega ; X)$ is dense in $H_{p, \Omega}^{\sigma}\left(\mathbb{R}^{n} ; X\right)$. From the imbedding theorem (cf. $\left.\S 8\right)$, we have

$$
\begin{aligned}
& W_{p}^{\infty}(\Omega ; X) \subset C^{\infty}(\Omega ; X), \\
& W_{p, \bar{\Omega}}^{\infty}\left(\mathbb{R}_{\mathbb{R}^{n}}^{n} ; X\right) \subset C_{\bar{\Omega}}^{\infty}\left(\mathbb{R}^{n} ; X\right) .
\end{aligned}
$$

Proof. It suffices to prove the denseness of $C_{0}^{\infty}(\Omega ; X)$. Since $V_{m}(t, x)$ is a $W_{p, \bar{\Omega}}^{\infty}\left(\mathbb{R}^{n} ; X\right)$-function with support contained in $\Omega$, taking $\varphi \in C_{0}^{\infty}\left(\boldsymbol{R}^{n}\right)$ such that

$$
\begin{array}{ll}
\varphi(x)=1 & \text { on }|x| \leqq 1, \\
\varphi(x)=0 & \text { in }|x|>2, \\
0 \leqq \varphi(x) \leqq 1, &
\end{array}
$$

it follows that

$$
g_{m \varepsilon}(x)=\varphi\left(\frac{x}{m}\right) V(\varepsilon, x) \rightarrow V(\varepsilon, x) \quad \text { in } B_{p, q}^{\sigma}\left(\mathbb{R}^{n} ; X\right)
$$

and that $g_{m \varepsilon} \in C_{0}^{\infty}(\Omega ; X)$.

Next, consider

Theorem 6. (Extension) By $R$ we shall denote the restriction to $\Omega$ of generalized functions defined on $\mathbb{R}^{n}$.

(I) There exist a bounded linear operator $E$ from $B_{p, q}^{\sigma}(\Omega ; X)$ into $B_{p, q}^{\sigma}\left(\mathrm{p}^{n} ; X\right)$ such that $R E f=f$ for any $f \in B_{p, q}^{\sigma}(\Omega ; X)$. More precisely, for any positive integers $i$ and $j$ there exists a linear operator $E$ :

$$
\bigcup_{-j<\sigma<i} B_{p, q}^{o}(\Omega ; X) \rightarrow \underset{-j \leq \sigma<i}{\bigcup} B_{p, q}^{\sigma}\left(\mathbb{R}^{n} ; X\right),
$$

whose restriction to $B_{p, q}^{\sigma}(\Omega ; X),-j<\sigma<i$, has the above properties.

(II) The same fact also holds for Sobolev spaces $H_{p}^{\sigma}(\Omega ; X)$ if we assume that $X$ is a Hilbert space and $1<p<\infty$.

Proof. (I) We shall use the integral representation given in Theorem 1 , in which we shall take $m, k, l, h$ so that $m-k \geqq j, l+k \geqq i, k \geqq i$, $l-h \geqq i, h+m \geqq j, h \geqq j(e . g . m=l=j+i, k=i, h=j)$. Let $u^{\alpha}, u_{\beta}, u_{\infty}^{\alpha}=$ 
$t^{|\alpha|} f_{\infty}^{(\alpha)}, f_{\beta}$ be as in Theorem 1 , and define

$$
\tilde{u}^{(\alpha)}(t, x)= \begin{cases}u^{\alpha}(t, x) & \text { for } x \in \Omega, \\ 0 & \text { for } x \notin \Omega .\end{cases}
$$

also define $\tilde{u}_{\beta}, \tilde{u}_{\infty}^{\alpha}, \tilde{f}_{\beta}, \tilde{f}_{\infty}$ analogously. If $f \in B_{p, q}^{\sigma}(\Omega: A),-j<\sigma<i$, then

$$
t^{-\sigma} \tilde{u}^{\alpha}, t^{-\sigma} \tilde{u}_{\beta}, t^{-\sigma} \tilde{u}_{\infty}^{\beta} \in L_{*}^{q}\left(I ; L^{p}\left(\underline{l}_{L^{\prime \prime}} ; X\right)\right)
$$

and $\tilde{f}_{\beta}, \tilde{f}_{\infty} \in L^{p}\left(\mathbb{R}^{p n} ; X\right)$. Hence the distribution $f(x)$ on $\mathbb{R}^{n}$ given by the formula for $f$ in Theorem 1 with $u^{\alpha}, u_{\beta}, t^{k} f_{\infty}^{(a)}, f_{\beta}$ and $f_{\infty}$ replaced by $\tilde{u}^{\alpha}, \tilde{u}_{\beta}, \tilde{u}_{\infty}^{\alpha}, \tilde{f}_{\beta}$ and $\tilde{f}_{\infty}$, respectively, belongs to $B_{p, q}^{\sigma}\left(\mathbb{R}^{n} ; X\right)$ and its restriction to $\Omega$ is equal to $f$. Moreover, it follows from Lemma 4.1, Lemma 3.3 and Lemma 4.2 that the mapping $f \rightarrow \tilde{f}$ is continuous form $B_{p, q}^{\sigma}(\Omega ; X)$ into $B_{p, q}^{\sigma}\left(\mathbb{R}^{n} ; X\right)$. This completes the proof of part (I). Part (II) can be proved similarly.

\section{§7 Imterpolation Theorems}

In this section we assume that $\sigma, \tau$ are real numbers, $1 \leqq p, q, \xi$, $\eta \leqq \infty$, and that $X$ is a Banach space.

To discuss the relation between Besov space $B_{p, q}^{\sigma}$ and Sobolev space $H_{p}^{\sigma}$, we first consider the following:

Lemma 7.1. Let $j$ be a positive integer such that $j \geqq 1+\sigma$, and let $1 \leqq \xi \leqq \eta \leqq \infty$. Assume that $1<p<\infty$ and that $X$ is reflexive.

(a) If $f \in W_{p}^{-\infty}(\Omega ; X)$ and if for any $\mathbb{K}(x, z) \in \mathscr{K}_{j}$

$$
\begin{aligned}
& t^{-\sigma} \int K(x,-z) f(x+t z) d z \in L^{p}\left(\Omega ; L_{*}^{\xi}(I ; X)\right), \\
&\left(o r \quad \in L_{*}^{\xi}\left(I ; L^{p}(\Omega ; X)\right)\right)
\end{aligned}
$$

then for any $\mathbb{K}(t, x, z, y) \in \mathscr{K}_{j}$

$$
\begin{array}{r}
t^{-\sigma} \int K(t, x,-z, x+t z) f(x+t z) d z \in L^{p}\left(\Omega ; L_{*}^{\eta}(I ; X)\right) . \\
\left(\text { or } \in \mathbb{L}_{*}^{\eta}\left(\mathbb{I} ; \mathbb{L}^{p}(\Omega ; X)\right)\right)
\end{array}
$$

(b) If $g \in W_{p, \bar{\Omega}}^{-\infty}\left(\mathbb{R}^{n} ; X\right)$ and if for any $K(x, z) \in \mathscr{K}_{j}$ 


$$
\begin{array}{r}
t^{-\sigma} \int K(x+t z, z) g(x+t z) d z \\
\in L^{p}\left(\Omega ; L_{*}^{\xi}(I ; X)\right), \\
\left(\text { or } \quad L_{*}^{\xi}\left(I ; L^{p}(\Omega ; X)\right)\right),
\end{array}
$$

then for any $K(t, x, z, y) \in \mathscr{K}_{j}$

$$
\begin{array}{r}
t^{-\sigma} \int K(t, x+t z, z, x) g(x+t z) d z \in L^{p}\left(\Omega ; L_{*}^{\prime \prime}(I ; X)\right) . \\
\left(o r \quad \in L_{*}^{\prime \prime}\left(I ; L^{p}(\Omega ; X)\right)\right) .
\end{array}
$$

Proof. By Lemma 3.4 we have

$$
\int K(t, x,-z, x+t z) f(x+t z) d z=U_{1}(t, x)+U_{2}(t, x)+U_{3}(t, x),
$$

where

$$
\begin{aligned}
& U_{1}(t, x)=\sum_{|\alpha| \leqq h}(-1)^{k-|\alpha|} \int \tilde{K}_{\alpha}(t, x,-z, x+t z) u^{\alpha}(t, x+t z) d z \\
& U_{2}(t, x)=\sum_{|\beta| \leqq h}(-1)^{|\beta|} \int t^{h-|\beta|} D_{z}^{\beta}\{K(t, x,-z, x+t z)\} u_{\beta}(t, x+t z) d z \\
& U_{3}(t, x)=\sum_{|\alpha| \leqq k}(-1)^{k-|\alpha|} \int \tilde{K}_{\alpha}(t, x,-z, x+t z) t^{k} f_{\infty}^{(\alpha)}(x+t z) d z .
\end{aligned}
$$

Here $u^{\alpha}, u_{\beta}, f_{\infty}$ are functions defined in Theorem 1 with $m=k=h, l=2 k$. Since $L^{(\alpha-\beta, \beta)} \in \mathscr{K}_{j}$, by the assumption we obtain

$$
s^{-\sigma} s^{k-|\beta|} \int L^{(a-\beta, \beta)}(x,-w) f(x+s w) d w \in L^{p}\left(\Omega ; L_{*}^{\xi}\right),
$$

so, with the aid of Lemma 3.3, we have $t^{-\sigma} u^{\alpha}(t, x) \in L^{p}\left(\Omega ; L_{*}^{\eta}\right)$. This and the following Lemma 7.3 gives

$$
t^{-\sigma} U_{1}(t, x) \in L^{p}\left(\Omega ; L_{*}^{\eta}\right)
$$

In the same way we obtain $t^{-\sigma} U_{2}(t, x) \in L^{p}\left(\Omega ; L_{*}^{\eta}\right)$. And $t^{-\sigma} U_{3}(t, x)$ $\in L^{p}\left(\Omega ; L_{*}^{\eta}\right)$ is obvious. Thus the lemma is proved.

Corolliary. If $1 \leqq \xi \leqq \eta \leqq \infty$, then

$$
B_{p, \xi}^{o}(\Omega ; X) \subset B_{p, \eta}^{\sigma}(\Omega ; X) .
$$


Proof. By Theorem 2 and the lemma we obtain the assertion.

Lemma 7.2. Let $X, Y$ be Banach spaces, $1<p$, and let $K(x, z, y)$ be an $\mathscr{L}(X, Y)$-valued strongly measurable function of $x, z, y \in \mathbb{R}^{n}$ which satisfies the inequality $(\mathscr{L}(X, Y)$ is the space of bounded linear operators $X \rightarrow Y)$

$$
\left\||z|^{n+1} D_{y}^{\alpha} D_{z}^{\beta} K(x, z, y)\right\|_{\mathscr{L}(X, Y)} \leqq C_{\alpha \beta}<\infty, \text { for }|\alpha|+|\beta|=1
$$

Then for all $f \in L^{p}\left(\mathbb{R}^{n} ; X\right) T f(x)=\int K(x, x-y, y) f(y) d y$ is convergent in $L^{p}\left(\mathbb{R}^{n} ; X\right)$ and

$$
\|T f\|_{L^{p}\left(\mathbb{R}^{n} ; X\right)} \leqq C_{p}\|f\|_{L^{p}\left(\mathbb{R}^{n} ; X\right)}
$$

if these facts hold for some $q$ with $1<p<q$.

Proof. By the same argument as in part (ii) of the proof of [21] Theorem 3 we have for any $f \in L^{1}\left(\mathbb{R}^{n} ; X\right)$

$$
\text { measure }\left\{x \mid\|T f(x)\|_{Y}>\lambda\right\} \leqq C \lambda^{-1}\|f\|_{L^{1}\left(\mathbb{R}^{n} ; X\right)} \text {. }
$$

From this and the Marcinkiewicz theorem (cf. [21] Theorem 2) the result follows.

Lemma 7.3. Let $K(t, x, z, y) \in \mathscr{K}_{0}$, and $1 \leqq p \leqq \infty, 1 \leqq \xi \leqq \infty$.

(I) If $u(t, x) \in L_{*}^{\xi}\left(I ; L^{p}(\Omega ; X)\right)$, then

$$
\begin{aligned}
& U(t, x)=\int K(t, x,-z, x+t z) u(t, x+t z) d z \in L_{*}^{\xi}\left(I ; L^{p}(\Omega ; X)\right), \\
& \widetilde{U}(t, x)=\int K(t, x+t z, z, x) u(t, x+t z) d z \in L_{*}^{\xi}\left(I ; L^{p}(\Omega ; X)\right) .
\end{aligned}
$$

(II) Assume one of the following conditions:

(i) $p=\xi$, (ii) $1<p \leqq \xi$, and (iii) $1<\xi \leqq p<\infty, X$ is reflexive. Then $U(t, x), \widetilde{U}(t, x) \in L^{p}\left(\Omega ; L_{*}^{\xi}(I ; X)\right)$ for any $u(t, x) \in L^{p}\left(\Omega ; L L_{*}^{\xi}(I ; X)\right)$.

Proof. It suffices to consider the case $\Omega=\mathbb{R}^{n}$.

(I).

$$
\left\|\int K(t, x,-z, x+t z) u(t, x+t z) d z\right\|_{L_{*}^{\xi}\left(I ; L^{p}\right)}
$$




$$
\begin{aligned}
& \leqq \int\|K(t, x,-z, x+t z) u(t, x+t z)\|_{L_{*}^{\xi}\left(I ; L^{p}\right)} d z, \\
& \leqq C \int_{b B} d z\left[\int_{0}^{a} d_{*} t\left\{\int\|u(t, x+t z)\|_{X}^{p} d x\right\}^{\xi / p}\right]^{1 / \xi}, \\
& =C a_{n} b^{n}\|u\|_{L_{*}^{\xi}\left(I ; L^{p}(\Omega ; X) .\right.}
\end{aligned}
$$

(II). (i) Case $p=\xi$. By (I), since $L_{*}^{\xi}\left(I ; L^{p}\right)=L^{p}\left(\Omega ; L_{*}^{p}\right)$.

(ii) Case $1<p \leqq \xi$. Since

$$
\int K(t, x,-z, x+t z) u(t, x+t z) d z=\int t^{-n} K\left(t, x, \frac{x-y}{t}, y\right) u(t, y) d y,
$$

the lemma in this case follows from Lemma 7.2 and the inequality

$$
\begin{aligned}
& \left\||z|^{n+1} D_{y}^{\alpha} D_{z}^{\beta} t^{-n} K\left(t, x, \frac{z}{t}, y\right)\right\|_{\mathscr{L}\left(L_{*}^{\xi}, L_{*}^{\xi}\right)} \\
& \leqq \sup _{a \geqq t>0}|z|^{n+1}\left|D_{y}^{\alpha} D_{z}^{\beta} t^{-n} K\left(t, x, \frac{z}{t}, y\right)\right|, \\
& \leqq a^{|\alpha|} \sup _{a \geqq t>0}|z|^{n+1} t^{-n-1}\left|K^{(0, \alpha, \beta)}\left(t, x, \frac{z}{t}, y\right)\right| \leqq C_{\alpha \beta} b^{n+1}<\infty,
\end{aligned}
$$

for $|\alpha|+|\beta|=1$. Here we have make use of the fact that $K^{(0, \alpha, \beta)}(t, x$, $z / t, y)=0$ for $|z|>t b$.

(iii) Case $1<\xi \leqq p<\infty$. Let $p^{\prime}$ and $\xi^{\prime}$ be the conjugate exponents of $p$ and $\xi$, respectivaly, and let $X^{\prime}$ be the dual space of $X$. For any $v(t, x)$ $\in L^{p^{\prime}}\left(\Omega ; L_{*}^{\xi^{\prime}}\left(I ; X^{\prime}\right)\right)$ set

$$
V(t, x)=\int K(t, x+t z, z, x) v(t, x) d x
$$

Notice that for $\varepsilon>0,0<\sigma<n$,

$$
\begin{aligned}
& \left\|\left\{\int_{\varepsilon}^{a}\|U(t, x)\| \xi_{X}^{\xi} d_{*} t\right\}^{1 / \xi}\right\| \|_{L^{p}\left(\mathbb{R}^{n}\right)} \\
& \quad \leqq C_{1} \varepsilon^{-\sigma}\left\|\int_{|x-y| \leqq b a} b^{n-\sigma}|x-y|^{\sigma-n}\right\| u(t, y)\left\|_{L_{*}^{\xi}} d y\right\|_{L^{p}\left(\mathbb{R}^{n}\right)}, \\
& \leqq C_{2} \varepsilon^{-\sigma} a^{\sigma}\|u\|_{L^{p}\left(\mathbb{R}^{n} ; L_{*}^{\xi}\right)}<\infty
\end{aligned}
$$


since

$$
\int_{|x-y| \leqq b a}|x-y|^{\sigma-n} d x=\int_{|x-y| \leqq b a}|x-y|^{\sigma-n} d y=\frac{n a_{n}}{\sigma} b^{\sigma} a^{\sigma}
$$

Thus we have by Fubini's theorem and Hölder's inequality

$$
\begin{aligned}
& \left|\int d x \int_{\varepsilon}^{a} U(t, x) v(t, x) d_{*} t\right| \\
& =\left|\int_{\varepsilon}^{a} d_{*} t \iint^{-n} K\left(t, x, \frac{x-y}{t}, y\right) u(t, y) v(t, x) d x d y\right| \\
& =\left|\int d y \int_{\varepsilon}^{a} V(t, y) u(t, y) d_{*} t\right|
\end{aligned}
$$

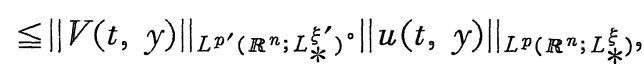

$$
\begin{aligned}
& \leqq C_{p^{\prime} \xi^{\prime}}\|v(t, x)\|_{\left.L^{p^{\prime}\left(\mathbb{R}^{n}\right.} ; L_{*}^{\xi^{\prime}}\right)^{\circ}}\|u(t, y)\|_{L^{p}\left(\mathbb{R}^{n} ; L_{*}^{\xi}\right)},
\end{aligned}
$$

here we have make use of the result in part (ii). From this and the duality (see [25])

$$
\left[L^{p}\left(\mathbb{R}^{n} ; L \stackrel{\xi}{\xi}(I ; X)\right)\right]^{\prime}=L^{p^{\prime}}\left(\mathbb{R}^{n} ; L_{*}^{\xi^{\prime}}\left(\mathbb{I} ; X^{\prime}\right)\right)
$$

the desired inequality follows, and the proof of the lemma completes.

We are now in a position to state the result concerning the comparison between the Besov spaces and Sobolev spaces:

Theomerm 7. Let $X$ be a Hilbert space. Then

(i) for $1<p \leqq 2$

$$
B_{p, p}^{\sigma}(\Omega ; X) \subset H_{p}^{\sigma}(\Omega ; X) \subset B_{p, 2}^{\sigma}(\Omega ; X)
$$

with continuous injections;

(ii) for $2 \leqq p<\infty$

$$
B_{p, 2}^{\sigma}(\Omega ; X) \subset H_{p}^{\sigma}(\Omega ; X) \subset B_{p, p}^{\sigma}(\Omega ; X)
$$

with continuous injections.

Proof. Let $j$ be a positive integer such that $j \geqq 1+\sigma$. Assume that $1<p \leqq 2, f \in B_{p, p}^{\sigma}(\Omega ; X)$. Then for any $K(x, z) \in \mathscr{K}_{j}$ 


$$
\begin{aligned}
t^{-\sigma} \int K(x,-z) f(x+t z) d z=t^{-\sigma} U(t, x) & \in L_{*}^{p}\left(I ; L^{p}(\Omega ; X)\right), \\
& =L^{p}\left(\Omega ; L_{*}^{p}(I ; X)\right),
\end{aligned}
$$

in view of Theorem 2. Therefore, from Lemma 7.1 it follows that $\iota^{-\sigma} U(t, x) \in L^{p}\left(\Omega ; L_{*}^{2}(I ; X)\right)$. From this and Theorem 2 it follows that $f \in H_{p}^{\sigma}(\Omega ; X)$.

Next assume that $f \in H_{p}^{\sigma}(\Omega ; X)$. Then we have $t^{-\sigma} U(t, x) \in L^{p}\left(\Omega ; L_{*}^{2}\right)$, which, with the help of Jessen's inequality, gives $t^{-\rho} U(t, x) \in L_{*}^{2}\left(I ; L^{p}(\Omega\right.$; $X))$. Thus, by Theoren 2 we obtain $f \in B_{p, 2}^{\sigma}(\Omega ; X)$. This gives the assertion of part (i).

The second assertion can be proved in the same way. If $2 \leqq p<\infty$, then by Lemma 7.1 and Jessen's inequality we have the fact that

$$
t^{-\sigma} U(t, x) \in L_{*}^{2}\left(I, L^{p}(\Omega ; X)\right)
$$

for any $K(x, z) \in \mathscr{K}_{j}$ implies

$$
t^{-\sigma} U(t, x) \in L^{p}\left(\Omega ; L_{*}^{2}(I ; X)\right)
$$

for any $K(x, z) \in \mathscr{K}_{j}$ and this implies

$$
t^{-\sigma} U(t, x) \in L^{p}\left(\Omega ; L_{*}^{p}\right)=L_{*}^{p}\left(I ; L^{p}(\Omega ; X)\right)
$$

for any $K \in \mathscr{K}_{j}$. From these facts and Theorem 2 we obtain the first assertion of part (ii).

This completes the proof of the theorem.

Lemma 7.4. Let $\sigma, \tau$ be real numbers with $\sigma \neq \tau$, and let $0<\theta<1$. Set $\ell=(1-\theta) \sigma+\theta \tau$. Then

$$
\left(L_{*^{\prime}}^{\infty, \sigma}(I ; X), L_{*}^{\infty, \tau}(I ; X)\right)_{\theta, q} \subset L_{*}^{q, \mu}(I ; X) \subset\left(L_{*}^{1, \sigma}(I ; X), L_{*}^{1, \tau}(I ; X)\right)_{\theta, q},
$$

with continuous injections, where

$$
L_{*}^{p, \sigma}(\mathbb{I} ; X)=L^{p}\left(I, t^{-\sigma p-1} d t ; X\right) .
$$

Proof. Let $f \in\left(L_{*}^{\infty, \sigma}, L_{*}^{\infty, \tau}\right)_{\theta, q}$. Then

$$
f(s)=\int_{0}^{\infty} u(t, s) d_{*} t
$$


with

$$
t^{\theta \lambda} u(t, s) \in L_{*}^{q}\left(\mathbb{R}^{+} ; L_{*^{\prime}, \sigma}^{\infty}\right), t^{\lambda(\theta-1)} u(t, s) \in L_{*}^{q}\left(\mathbb{R}^{+} ; L_{*^{*}, \tau}^{\infty}\right),
$$

where $\lambda=\sigma-\tau$. Consider the case $\lambda>0$. Setting

$$
f_{1}(s)=\int_{s}^{\infty} u(t, s) d_{*} t, f_{2}(s)=\int_{0}^{s} u(t, s) d_{*} t
$$

we have, by $[20]$ Lemma 2.5,

$$
\begin{aligned}
\left\|s^{-\mu} f_{1}(s)\right\|_{L_{*}^{q}(I ; X)} & \leqq \|\left[\int_{s}^{\infty} t^{-\lambda \theta} s^{\lambda \theta}\left\|t^{\lambda \theta} s^{-\sigma} u\left(t, s \|_{L_{*}^{\infty}(I ; X)} d_{*} t\right]\right\|_{L_{*}^{q}(I)},\right. \\
& \leqq \frac{1}{\lambda \theta}\left\|t^{\lambda \theta} u(t, s)\right\|_{L_{*}^{q}\left(\boldsymbol{R}^{+} ; L_{*}^{\infty}, \sigma\right)}
\end{aligned}
$$

since

$$
\int_{s}^{\infty} t^{-\lambda \theta} s^{\lambda \theta} d_{*} t=\frac{1}{\lambda \theta}, \quad \int_{0}^{t} t^{-\lambda \theta} s^{\lambda \theta} d_{*} s=\frac{1}{\lambda \theta} .
$$

Similarly, we obtain

$$
\left\|s^{-\mu} f_{2}(s)\right\|_{L_{*}^{q}(I ; X)} \leqq \frac{1}{\lambda(1-\theta)}\left\|t^{\lambda(\theta-1)} u(t, s)\right\|_{L_{*}^{q}\left(\boldsymbol{R}^{+} ; L_{*^{\prime}}^{\infty} \tau\right)}
$$

and, therefore, we have $f \in L_{*}^{q, \mu}(I ; X)$. The proof for the case $\lambda<0$ goes the same way.

Conversely let $f \in L_{*}^{q, \mu}(I ; X)$ and assume that $\lambda>0$. Set

$$
\begin{aligned}
& v(t, s)= \begin{cases}f(s) & \text { for } s>t \\
0 & \text { otherwise }\end{cases} \\
& w(t, s)=f(s)-v(t, s) .
\end{aligned}
$$

Then, by $[20]$ lemma 2.5 we obtain

$$
\begin{aligned}
& \left\|t^{\lambda \theta}\right\| s^{-\sigma} v(t, s)\left\|_{L_{*}^{1}(I ; X)}\right\|_{L_{*}^{q}\left(\boldsymbol{R}^{+}\right)} \\
\leqq & \left\|\int_{t}^{a}\left\{s^{-\mu}\|f(s)\|_{X}\right\} s^{-\lambda \theta} t^{\lambda \theta} d_{*} s\right\|_{L_{*}^{q}\left(\boldsymbol{R}^{+}\right)} \leqq \frac{1}{\lambda \theta}\|f\|_{L_{*}^{q}, \mu(I ; X)}
\end{aligned}
$$


and

$$
\left\|t^{\lambda(\theta-1)}\right\| s^{-\tau} w(t, s)\left\|_{L_{*}^{1}(I ; X)}\right\|_{L_{*}^{q}\left(\boldsymbol{R}^{+}\right)} \leqq \frac{1}{\lambda(1-\theta)}\|f\|_{L_{*}^{q, \mu}(I ; X)}
$$

Hence $f \in\left(L_{*}^{1}, \sigma, L_{*}^{1}, \tau\right)_{\theta, q}$, and the proof of the lemma completes.

\section{Lemma 7.5.}

$$
B_{p, 1}^{\sigma}(\Omega ; X) \subset H_{p}^{\sigma}(\Omega ; X) \subset B_{p, \infty}^{\sigma}(\Omega ; X)
$$

with continuous injections.

Proof. First consider the case where $\sigma$ is a non-negative integer $k$. It follows from Lemma 3.1 Corollary 1 that

$$
\begin{aligned}
f(x) & =\int_{0}^{a} d_{*} t \int M(x,-z) f(x+t z) d z+f_{\infty}(x), \\
f_{\infty}(x) & =\int \omega_{m}(x,-z) f(x+a z) d z .
\end{aligned}
$$

Assume that $f \in B_{p, 1}^{k}(\Omega ; X)$. Then, by Theorem 2 we have

$$
\begin{aligned}
& D_{x}^{\alpha} \int M(x,-z) f(x+t z) d z \\
& \quad=\sum_{\beta}\left(\begin{array}{c}
\alpha \\
\beta
\end{array}\right) t^{-|\beta|} \int M^{(\alpha-\beta, \beta)}(x,-z) f(x+t z) d z \in L_{*}^{1}\left(I ; L^{p}\right)
\end{aligned}
$$

for any $|\alpha| \leqq k$. Therefore $f \in W_{p}^{k}$. Second assume that $f \in W_{p}^{k}$. Then, for any $K \in \mathscr{K}_{k+1}$ we have

$$
t^{-k} \int K(x,-z) f(x+t z) d z=\sum_{|\alpha|=k} \int K_{\alpha}(x,-z) f^{(\alpha)}(x+t z) d z \in L_{*}^{\infty}\left(I ; L^{p}\right)
$$

so we obtain $f \in B_{p, \infty}^{k}$ by Theorem 2 .

Next consider the case where $\sigma$ is a negative integer $-k$. Assume that $f \in B_{p, 1}^{-k}$. Then

$$
f=\sum_{|\alpha| \leqq k} D^{\alpha} f_{\alpha}, f_{\alpha} \in B_{p, 1}^{0}
$$

Since $B_{p, 1}^{0} \subset L^{p}$, we obtain $f \in W_{p}^{-k}$. In the same way we have $W_{p}^{-k} \subset B_{p, \infty}^{-k}$. 
Finally consider the case where $\sigma$ is a fractional number. From the first identity of Theorem 8 below and Lions-Peetre [17] Theorem IV 1.4 it follows that

$$
\begin{aligned}
B_{p, 1}^{\sigma}=\left(B_{p, 1}^{k}, B_{p, 1}^{k+1}\right)_{\theta, 1} & \subset\left(W_{p}^{k}, W_{p}^{k+1}\right)_{\theta, 1} \subset\left[W_{p}^{k}, W_{p}^{k+1}\right]_{\theta} \\
= & H_{p}^{\sigma} \subset\left(W_{p}^{k}, W_{p}^{k+1}\right)_{\theta, \infty} \subset\left(B_{p, \infty}^{k}, B_{p, \infty}^{k+1}\right)_{\theta, \infty}=B_{p, \infty}^{\sigma} .
\end{aligned}
$$

Now, we shall discuss the interpolation space of the Besov spaces and Sobolev spaces.

Theorem 8. (Interpolation spaces). Let $0<\theta<1$ and set $\mu=(1-\theta) \sigma+\theta \tau, \frac{1}{r}=\frac{1-\theta}{p}+\frac{\theta}{q}, \frac{1}{\zeta}=\frac{1-\theta}{\xi}+\frac{\theta}{\eta}$.

$$
\begin{aligned}
& \left(B_{p, \xi}^{\sigma}(\Omega ; X), B_{p, \eta}^{\tau}(\Omega ; X)\right)_{\theta, q}=B_{p, q}^{\mu}(\Omega ; X), \\
& \left(H_{p}^{\sigma}(\Omega ; X), H_{p}^{\tau}(\Omega ; X)\right)_{\theta, q}=B_{p, q}^{\mu}(\Omega ; X),
\end{aligned}
$$

with equivalent norms. Here we assume that $\sigma \neq \tau$.

$$
\begin{aligned}
& {\left[B_{p, \xi}^{\sigma}(\Omega), B_{q, \eta}^{\tau}(\Omega)\right]_{\theta}=B_{r, \zeta}^{\mu}(\Omega),} \\
& {\left[H_{p}^{\sigma}(\Omega), H_{q}^{\tau}(\Omega)\right]_{\theta}=H_{r}^{\mu}(\Omega),}
\end{aligned}
$$

with continuous injections. In the second assertion we assume that $1<r<\infty$.

Proof. Let $j>\sigma, \tau$ be a non-negative integer, and let $K(x, z) \in \mathscr{K}_{j}$. Then

$$
U(t, x)=\int K(x,-z) f(x+t z) d z
$$

is a bounded linear operator from $B_{p, \infty}^{\sigma}$ into $L_{*^{\prime}}^{\infty} \sigma\left(I ; L^{p}(\Omega ; X)\right.$ ), also from $B_{p, \infty}^{\tau}$ into $L_{*}^{\infty, \tau}\left(I ; L^{p}(\Omega ; X)\right)$. Therefore, by the interpolation of operators we obtain it is a bounded linear operator from $\left(B_{p, \infty}^{\sigma}, B_{p, \infty}^{\tau}\right)_{\theta, q}$ into $\left(L_{*}^{\infty, \sigma}(I\right.$; $\left.L^{p}(\Omega ; X)\right), L_{*}^{\infty}{ }^{\top}\left(I ; L^{p}(\Omega ; X)\right)_{\theta, q}$. This, with the help of Lemma 7.4 , gives $t^{-\mu} U(t, x) \in \mathbb{L}_{*}^{q}\left(I ; L^{p}(\Omega)\right)$ for every $f \in\left(B_{p, \infty}^{\sigma}, B_{p, \infty}^{\tau}\right)_{\theta, q}$. Thus, using Theorem 2, we have $\left(B_{p, \infty}^{\sigma}, B_{p, \infty}^{\tau}\right)_{\theta, q} \subset B_{p, q}^{\mu}$. In the same way by Lemma 7.4, Theorem 1, Theorem 2, Lemma 4.2 and the interpolation of operators we have $\left(B_{p, 1}^{\sigma}, B_{p, 1}^{\tau}\right)_{\theta, q} \supset B_{p, q}^{\mu}$. Combining these facts with Lemma 7.1 Corollary 
we obtain the first assertion.

The second identity follows from the first and Lemma 7.5.

(ii). By the facts that

$$
\begin{aligned}
& \left.\left[L_{*}^{\xi}, \sigma\left(I ; L^{p}(\Omega)\right), L_{*}^{\eta}{ }^{\top}\left(\mathbb{I}, L^{q}\right)\right)\right]_{\theta}=L_{*}^{\xi}{ }^{\mu}\left(\mathbb{I} ; L^{r}(\Omega)\right), \\
& {\left[L^{p}\left(\Omega ; L_{*}^{\xi}, \sigma\right), L^{q}\left(\Omega ; L_{*}^{\eta, \tau}\right)\right]_{\theta}=\mathbb{L}^{r}\left(\Omega ; L_{*^{\zeta}}^{\zeta} \mu\right),}
\end{aligned}
$$

(cf. Calderón [8]) and by Theorem 2 we obtain the assertions, with the aid of the interpolation of operators.

Remark. we may discuss the interpolation space in another situations, but shall not develop here. See [18], [20].

By Theorem 8, Theorem 6 and the fact that $H_{p}^{\sigma}\left(\mathbb{R}^{n}\right)$ coincides with the space of generalized functions such that

$$
F^{-1}\left\{(1+|\xi|)^{\sigma} F f(\xi)\right\} \in L^{p}\left(\mathbb{R}^{n}\right),
$$

where $F$ denotes Fourier transform, (see Calderóns [8], Schechter [27], Lions [15]), we obtain.

Corrollary. The space $H_{p}^{\sigma}(\Omega), 1<p<\infty$, coincides with the space of generalized functions $f$ such that there exists a generalized function $\tilde{f}$ satisfying (7.1) with $\tilde{f}=f$ in $\Omega$. The norm of $H_{p}^{\sigma}(\Omega)$ is equivalent with

$$
\inf \left\|F^{-1}\left((1+|\xi|)^{\sigma} F \tilde{f}(\xi)\right)\right\|_{L^{p}\left(\mathbb{R}^{n}\right)},
$$

where the infimum is taken over all such $\tilde{f}$.

\section{§o Imbedding 'Thherrerms.}

We shal] start with

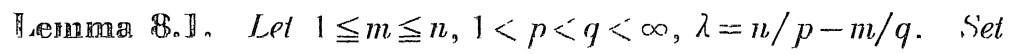

$$
F(x)=\int|x-y|^{-n+\lambda} f(y) d y
$$

for $f \in \mathbb{L}^{p}\left(\mathbb{R}^{n}\right)$. Then

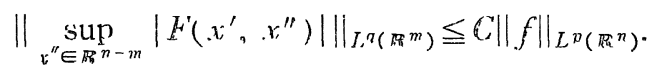


Proof. Since

$$
|F(x)| \leqq \int d y^{\prime}\left\{\int|x-y|^{(-n+\lambda) p^{\prime}} d y^{\prime \prime}\right\}^{1 / p^{\prime}}\left\|f\left(y^{\prime}, y^{\prime \prime}\right)\right\|_{L^{p}\left(\boldsymbol{R}^{n-m}\right)},
$$

where $p^{\prime}$ is the conjugate exponent of $p$. Write $\rho=\left|x^{\prime}-y^{\prime}\right|, \rho t=\left|x^{\prime \prime}-y^{\prime \prime}\right|$, $2 \sigma=(\lambda-n) p^{\prime}$. Then

$$
\begin{array}{r}
\int|x-y|^{p^{\prime}(-n+\lambda)} d y^{\prime \prime}=(n-m) a_{n-m} \rho^{2 \sigma+n-m} \int_{0}^{\infty}\left(1+t^{2}\right)^{\sigma} t^{n-m} d_{*} t \\
\leqq C_{\sigma, n, m} \rho^{2 \sigma+n-m},
\end{array}
$$

where $a_{n}$ is the volume of the unit ball in $\mathbb{R}^{n}$. Hence we have

$$
\sup _{x^{\prime \prime}}\left|F\left(x^{\prime}, x^{\prime \prime}\right)\right| \leqq C \int\left|x^{\prime}-y^{\prime}\right|^{\lambda^{\prime}-m} g\left(y^{\prime}\right) d y^{\prime}
$$

where

$$
g\left(y^{\prime}\right)=\left\|f\left(y^{\prime}, y^{\prime \prime}\right)\right\|_{L^{p}\left(\boldsymbol{R}^{n-m}\right)}, \lambda^{\prime}=\frac{m}{p}-\frac{m}{q} .
$$

From this and Hardy-Littlewood-Sobolev's inequality the desired inequality follows.

Lemma 8.2. Let $1 \leqq m \leqq n, 1 \leqq p \leqq q \leqq \infty, \lambda=\frac{n}{p}-\frac{m}{q}, \varepsilon>0$. Set

$$
F(x)=\int_{|x-y| \leqq d}|x-y|^{-n+\lambda+\varepsilon} f(y) d y
$$

for $f \in L^{p}\left(\mathbb{R}^{n}\right)$. Then

$$
\left\|\sup _{x^{\prime \prime} \in \mathbb{R}^{n-m}} \mid F\left(x^{\prime}, x^{\prime \prime}\right)\right\|\left\|_{L^{q}\left(\boldsymbol{R}^{m}\right)} \leqq C\right\| f \|_{L^{p}\left(\mathbb{R}^{n}\right)} .
$$

Proof. In the same way as in the proof of Lemma 8.1 we have

$$
\sup _{x^{\prime \prime}}\left|F\left(x^{\prime}, x^{\prime \prime}\right)\right| \leqq C \int_{\left|x^{\prime}-y^{\prime}\right| \leqq d}\left|x^{\prime}-y^{\prime}\right|^{-m+\lambda^{\prime}+\varepsilon} g\left(y^{\prime}\right) d y^{\prime}
$$

Let $r$ be a real number such that $1 / p-1 / q=1-1 / r$. Then

$$
\begin{aligned}
\int_{\left|x^{\prime}-y^{\prime}\right| \leqq d}\left|x^{\prime}-y^{\prime}\right|^{\left(-m+\lambda^{\prime}+\varepsilon\right) r} d y^{\prime} & =\int_{\left|x^{\prime}-y^{\prime}\right| \leqq d}\left|x^{\prime}-y^{\prime}\right|^{\left(-m+\lambda^{\prime}+\varepsilon\right) r} d x^{\prime} \\
& =m a_{m} d^{\varepsilon r} / \varepsilon r
\end{aligned}
$$


so we have the assertion with the aid of [20] Lemma 2.5.

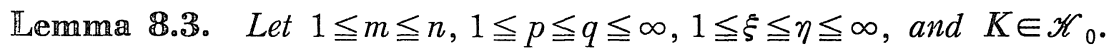
Assume that $u \in L^{p}\left(\Omega ; L_{*}^{\eta}(I)\right)$.

(i) If $1<p<q<\infty$, then

$$
\begin{gathered}
\sup _{x^{\prime \prime} \in \mathbb{R}^{n-m}}\left\|\left[\left\|t^{\lambda} \int K(x,-z) u(t, x+t z) d z\right\|_{L_{*}^{\xi}}\right]\right\|_{L^{q}\left(\Omega^{\prime}\left(x^{\prime \prime}\right)\right)} \\
\leqq C\|u(t, x)\|_{L^{p}\left(\Omega ; L_{*}^{\eta}\right)},
\end{gathered}
$$

where $\Omega^{\prime}\left(x^{\prime \prime}\right)=\left\{x^{\prime} \mid\left(x^{\prime}, x^{\prime \prime}\right) \in \Omega\right\}$.

(ii) If $\varepsilon>0$, then

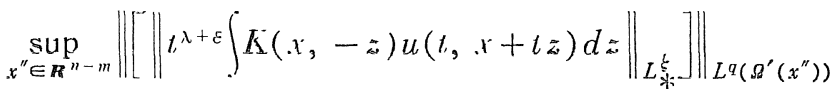

$$
\begin{aligned}
& \leqq C\|u(t, x)\|_{L^{p}\left(\Omega ; L_{*}^{\eta}\right)} .
\end{aligned}
$$

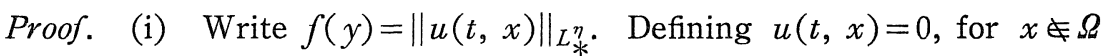
we may consider only the case $\Omega=\mathbb{R}^{n}$. Let $r=\eta / \xi$, and let $r^{\prime}$ be the conjuagte exponent of $r$. Then, from Jessen's inequality and Hölder's inequality, it follows that

$$
\begin{aligned}
\left\|t^{\lambda} \int K(x,-z) u(t, x+t z) d z\right\| L_{*}^{\xi} & \leqq C \int\left\|t^{\lambda-n} K\left(x, \frac{x-y}{t}\right)\right\| L_{*}^{\xi} r^{\prime} f(y) d y \\
& \leqq C \int_{|x-y| \leqq a b}|x-y|^{\lambda-n} f(y) d y,
\end{aligned}
$$

which, combined with Lemma 8.1, gives the conclusion.

Similarly for part (ii).

Theorem 9. (Imbedding) Let $1 \leqq p \leqq q \leqq \infty, 1 \leqq \xi, \eta \leqq \infty, \lambda=\frac{n}{p}-\frac{n}{q}$, $\varepsilon>0$, and let $\sigma$ be a real number. Then the following imbedding operators exist under the additional assumptions:

$$
\begin{array}{ll}
\text { B-B (i) } & B_{p, \xi}^{\sigma+\lambda+\varepsilon}(\Omega) \rightarrow B_{q, \eta}^{\sigma}(\Omega), \\
\text { B-B (ii) } & B_{p, \xi}^{\sigma+\lambda}(\Omega) \rightarrow B_{q, \eta}^{\sigma}(\Omega),(\xi \leqq \eta), \\
\text { B-H (i) } & B_{p, \xi}^{\sigma+\lambda+\varepsilon}(\Omega) \rightarrow H_{q}^{\sigma}(\Omega),
\end{array}
$$


$\mathrm{B}-\mathrm{H}(\mathrm{ii}) \quad B_{p, \xi}^{\sigma+\lambda}(\Omega) \rightarrow H_{q}^{\sigma}(\Omega), \quad(p<q, \xi \leqq q<\infty)$,

$\mathrm{H}-\mathrm{B}(\mathrm{i}) \quad H_{p}^{\sigma+\lambda+\varepsilon}(\Omega) \rightarrow B_{q, \eta}^{\sigma}(\Omega)$,

$\mathrm{H}-\mathrm{B}(\mathrm{ii}) \quad H_{p}^{\sigma+\lambda}(\Omega) \rightarrow B_{q, \eta}^{\sigma}(\Omega), \quad(1<p<q, p \leqq \eta)$

$\mathrm{H}-\mathrm{H}(\mathrm{j}) \quad H_{p}^{\sigma+\lambda+\varepsilon}(\Omega) \rightarrow H_{p}^{\sigma}(\Omega)$,

$\mathrm{H}-\mathrm{H}(\mathrm{ii}) \quad H_{p}^{\sigma+\lambda}(\Omega) \rightarrow H_{q}^{\sigma}(\Omega) . \quad(1<p<q<\infty)$.

Proof. B-B. By $[20]$ Theorem 2, and Theorem 3 Corollary 1.

$\mathrm{B}-\mathrm{H}$. For the case where $\sigma$ is an integer the assertion follows from [20] Theorem 2 and Theorem 3 Corollary 1 . The proof of the case where $\sigma$ is fractional follows from the above result and the interpolation.

$\mathrm{H}-\mathrm{B}$. In the same way as for $\mathrm{B}-\mathrm{H}$.

$\mathrm{H}-\mathrm{H}$ (i). This follows from $\mathrm{B}-\mathrm{H}$ (i) and $\mathrm{H}-\mathrm{B}$ (i).

$\mathrm{H}-\mathrm{H}$ (ii). Let $i$ be a non-negative integer with $1+\sigma<i$. Assume that $K(x, z)=\sum_{|\alpha|=i} K_{a}^{(0, \alpha)}(x, z) \in \mathscr{K}_{i}$. Then, from Lemma 3.4 it follows that

$$
\int K(x,-z) f(x+t z) d z=U(t, x)=U_{1}(t, x)+U_{2}(t, x)+U_{3}(t, x),
$$

where

$$
\begin{aligned}
& U_{1}(t, x)=\sum_{|\alpha|=i} \int K_{\alpha}(x,-z) u^{\alpha}(t, x+t z) d z \\
& U_{2}(t, x)=\sum_{|\beta| \leqq h} t^{h-|\beta|} \int K^{(0, \beta)}(x,-z) u_{\beta}(t, x+t z) d z \\
& U_{3}(t, x)=\sum_{|\alpha|=i} l^{i} \int K_{\alpha}(x,-z) f_{\infty}^{(a)}(x+t) d z .
\end{aligned}
$$

Since by $\mathrm{H}-\mathrm{B}$ (i) we have $f_{\infty} \in \mathbb{W}_{p}^{\infty} \subset \mathbb{W}_{q}^{\infty}$, we obtain

$$
\iota^{-\sigma} U_{3}(t, x) \in L^{q}\left(\Omega ; L_{*}^{2}(I)\right) .
$$

Also we have, by Theorem 2 and Lemma 3.3,

$$
t^{-\lambda-\sigma} u^{\alpha}, t^{-\lambda-\sigma} u_{\beta} \in L^{p}\left(\Omega ; L_{*}^{2}\right)
$$

so that by Lemma 8.3 we obtain 


$$
t^{-\sigma}\left(U_{1}(t, x)+U_{2}(t, x)\right) \in L^{q}\left(\Omega ; L_{*}^{2}\right)
$$

Thus, combining this with the fact $f \in W_{p}^{-\infty} \subset W_{q}^{-\infty}$, which follows from $\mathrm{B}-\mathrm{H}$ (i), we obtain $f \in H_{q}^{\sigma}$.

Next we shall study the trace operator to any hyperplane. To do this we need the following:

Lemmant \&.4. Let $1 \leqq m<n, 1<p \leqq \eta, p \leqq \eta$, and lot $u(l, x) \in L^{p}(\Omega$; $\left.L_{*}^{\infty}(\mathbb{I})\right) . \quad$ Definc

$$
U(t, x)=\int_{b B} x(x+t z)|u(t, x+t \tilde{w})| d \approx
$$

where $x$ is the characteristic function of $\Omega$. Then

$$
\sup _{x^{\prime \prime} \in \mathbb{R}^{n-m}}\left\|t^{(n-m) / p} U\left(t, x^{\prime}, x^{\prime \prime}\right)\right\|_{L_{*}^{\eta}\left(I ; L^{q}\left(\Omega^{\prime}\left(x^{\prime \prime}\right)\right)\right.} \leqq C\|u\|_{L^{p}\left(\Omega ; L_{*}^{\infty}\right)},
$$

where $\Omega^{\prime}\left(x^{\prime \prime}\right)=\left\{x^{\prime} \mid\left(x^{\prime}, x^{\prime \prime}\right) \in \Omega\right\}$.

Proof. Write $f(x)=\|u(t, x)\|_{L_{*}^{\infty} \cdot}$ Define $f(x)=0$ for $x \notin \Omega$. Then

$$
\begin{aligned}
|U(t, x)| & \leqq C_{1} \int_{|z| \leqq b}|f(x+t z)| d z, \\
& =C_{1} \int_{\left|z^{\prime \prime}\right| \leqq b} d z^{\prime \prime} \int_{\left|x^{\prime}-y^{\prime}\right| \leqq t b} t^{-m} \mid f\left(y^{\prime}, x^{\prime \prime}+t z^{\prime \prime}\right) d y^{\prime},
\end{aligned}
$$

From $\left[20^{-}\right]$Lemma 2.5 and

$$
\int_{1^{\prime} v^{\prime} \mid \leq t b} d y^{\prime}=\int_{\left.\right|^{\prime}-y^{\prime} \leq t b} d x^{\prime}=a_{m} b^{m} t^{m}
$$

it follows that

$$
\begin{aligned}
\left\|U\left(t, x^{\prime}, x^{\prime \prime}\right)\right\|_{L^{\prime \prime}\left(\Omega^{\prime}\left(x^{\prime \prime}\right)\right)} & =C_{i} \int_{\left|z^{\prime \prime}\right| \leq b} g\left(.1^{\prime \prime}+t z^{\prime \prime}\right) d z_{z^{\prime \prime}}, \\
& =C_{c_{2}} \iota^{m} n \int_{\left|u^{\prime \prime}\right| \leqq t b} g\left(x^{\prime \prime}+u^{\prime \prime}\right) d u^{\prime \prime},
\end{aligned}
$$

where

$$
g\left(x^{\prime \prime}\right)=\left\|f\left(1^{\prime}, x^{\prime \prime}\right)\right\|_{L^{\prime \prime}\left(m^{\prime \prime \prime}\right)} .
$$


This gives

$$
\begin{aligned}
& \left\|t^{\frac{n-m}{p}} U(t, x)\right\|_{L_{*}^{\eta}\left(I ; L^{q}\left(\Omega^{\prime}\left(x^{\prime \prime}\right)\right)\right)} \\
& \quad \leqq\left\|\int_{\left|u^{\prime \prime}\right| \leqq t b} t^{-\sigma}\left|u^{\prime \prime}\right| \sigma\left\{g\left(x^{\prime \prime}+u^{\prime \prime}\right)\left|u^{\prime \prime}\right|^{\frac{n-m}{p}}\right\} \frac{d u^{\prime \prime}}{\left|u^{\prime \prime}\right|^{n-m}}\right\|_{L_{*}^{\eta}(I)} \\
& \quad \leqq C\|u(t, x)\|_{L^{p}\left(\Omega ; L_{*}^{\infty}(I)\right)},
\end{aligned}
$$

where $\sigma=(n-m)(1-1 / p)$, since

$$
\begin{aligned}
& \int_{\left|u^{\prime \prime}\right| \leqq b t} t^{-\sigma}\left|u^{\prime \prime}\right| \sigma \frac{d u^{\prime \prime}}{\left|u^{\prime \prime}\right|^{n-m}}=\frac{n-m}{\sigma} a_{n-m} b^{n-m}, \\
& \int_{\frac{\mid u^{\prime \prime}}{b} t^{-\sigma}\left|u^{\prime \prime}\right|^{\sigma} d_{*} t=\frac{b^{\sigma}}{\sigma}}^{\infty} \\
& \left|u^{\prime \prime}\right|{ }^{\sigma} t^{-\sigma} \leqq b^{\sigma} \text { for } \quad\left|u^{\prime \prime}\right| \leqq b t .
\end{aligned}
$$

This establishes the lemma.

Lemma 8.5. Let $1 \leqq m<n, \Omega \subset \mathbb{R}^{n}, 1<p \leqq q, p \leqq \eta$, and $\lambda=n / p-m / q$. Assume that $\sigma>0, K(x, z) \in \mathscr{K}_{0}$,

$$
t^{-\sigma-\lambda} u(t, x) \in L^{p}\left(\Omega ; L_{*}^{\infty}(I)\right),
$$

and define

$$
F(x)=\int_{0}^{a} d_{*} t \int K(x,-z) u(t, x+t z) d z
$$

Then

$$
\sup _{x^{\prime \prime} \in \mathbb{R}^{n-m}}\left\|F\left(x^{\prime}, x^{\prime \prime}\right)\right\|_{B_{q, \eta}^{\sigma}\left(\Omega^{\prime}\left(x^{\prime \prime}\right)\right)} \leqq C\left\|t^{-\sigma-\lambda} u\right\|_{L^{p}\left(\Omega ; L_{*}^{\infty}(I)\right)}
$$

Proof. Let $\sigma=k+\theta$, where $0<\theta \leqq 1, k$ is a non-negative integer. First consider the case $k=0$.

For any $x \in \Omega_{2, y}=(\Omega-2 y) \cap(\Omega-y) \cap \Omega$ we get

$$
\begin{aligned}
& |F(x+2 y)-2 F(x+y)+F(y)| \\
= & \mid \int_{0}^{a} t^{-n} d_{*} t\left\{\left\{\sum_{j=0}^{2}(-1)^{j}\left(\begin{array}{l}
2 \\
j
\end{array}\right) K\left(x+j y, \frac{x+j y-w}{t}\right)\right\} u(t, w) d w \mid,\right.
\end{aligned}
$$




$$
\begin{aligned}
& \leqq C_{1} \sum_{j=0}^{2} \int_{0}^{a} t^{-n} d_{*} t \int_{C(t, x+j y)}\left\{h\left(\frac{|y|}{t}\right)^{2}+h(|y|)^{2}\right\}|u(t, w)| d w, \\
& =C_{1} \sum_{j=0}^{2} \int_{0}^{a}\left\{h\left(\frac{|y|}{t}\right)^{2}+h(|y|)^{2}\right\} U(t, x+j y) d_{*} t,
\end{aligned}
$$

where $h(t)=\min \{t, 1\}, C(t, x)=\{y=x+t z \mid z \in A(t, x)\} \subset \Omega \cap\{x+t b B\}$,

$$
U(t, x)=\int_{b B \cap t^{-1}(\Omega-x)}|u(t, x+t z)| d z
$$

Therefore, for $y^{\prime} \in \mathbb{R}^{m}$ we obtain

$$
\begin{aligned}
\varphi\left(y^{\prime}\right) & =\left\|F\left(x^{\prime}+2 y^{\prime}, x^{\prime \prime}\right)-2 F\left(x^{\prime}+y^{\prime}, x^{\prime \prime}\right)+F\left(x^{\prime}, x^{\prime \prime}\right)\right\|_{L^{q}\left(\Omega^{\prime} 2, y^{\prime}\left(x^{\prime \prime}\right)\right)} \\
& \leqq 3 C_{1} \int_{0}^{a}\left\{h\left(\frac{\left|y^{\prime}\right|}{t}\right)^{2}+h\left(\left|y^{\prime}\right|\right)^{2}\right\}\left\|U\left(t, x^{\prime}, x^{\prime \prime}\right)\right\|_{L^{q}\left(\Omega^{\prime}\left(x^{\prime \prime}\right)\right)} d_{*} t .
\end{aligned}
$$

But

$$
\begin{aligned}
& \int_{0}^{a} h\left(\frac{\left|y^{\prime}\right|}{t}\right)^{2} t^{\theta}\left|y^{\prime}\right|^{-\theta} d_{*} t \leqq \frac{2}{(2-\theta) \theta}, \\
& \int h\left(\frac{\left|y^{\prime}\right|}{t}\right)^{2} t^{\theta}\left|y^{\prime}\right|^{-\theta} d_{*} y^{\prime}=\frac{2 m a_{m}}{(2-\theta) \theta}, \\
& \int_{0}^{a} h\left(\left|y^{\prime}\right|\right)^{2} t^{\theta}\left|y^{\prime}\right|^{-\theta} d_{*} t \leqq \frac{a^{\theta}}{\theta} \\
& \int h\left(\left|y^{\prime}\right|\right)^{2} t^{\theta}\left|y^{\prime}\right|^{-\theta} d_{*} y^{\prime}=\frac{2 m a_{m} t^{\theta}}{(2-\theta) \theta} \leqq \frac{2 m a_{m} a^{\theta}}{(2-\theta) \theta} \quad \text { for } t \leqq a .
\end{aligned}
$$

From this and $[20]$ Lemma 2.5 it follows that

$$
\left\|F\left(x^{\prime}, x^{\prime \prime}\right)\right\|_{B_{q, \eta}^{\theta}} \leqq C_{2}\left\|t^{-\theta} U\left(t, x^{\prime}, x^{\prime \prime}\right)\right\|_{L_{*}^{\eta}\left(I ; L^{q}\left(\Omega^{\prime}\left(x^{\prime \prime}\right)\right)\right.},
$$

where $C_{2}$ is a constant independent of $f$ and $x^{\prime \prime}$, and the desired estimate follows from this and Lemma 8.4.

Case $k>0$. Let $|\alpha| \leqq k$. Then

$$
\begin{aligned}
D_{x}^{\alpha} F(x) & =D_{x}^{\alpha} \int_{0}^{a} d_{*} t \int K(x,-z) u(t, x+t z) d z, \\
& =\sum_{\beta}\left(\begin{array}{c}
\alpha \\
\beta
\end{array}\right) \int_{0}^{a} t^{-|\beta|} d_{*} t \int K^{(\alpha-\beta, \beta)}(x,-z) u(t, x+t z) d z,
\end{aligned}
$$


so that the assertions for the present case follows from this and the result for the case $k=0$.

This completes the proof of the lemma.

The result for the existence of the trace operator (the restriction mapping) to any hyperplane is as follows;

The

$$
\Omega \subset \mathbb{R}^{n}, 1 \leqq m<n, x^{\prime \prime} \in \mathbb{R}^{n-m}, \Omega^{\prime}=\Omega^{\prime}\left(x^{\prime \prime}\right)=\left\{x^{\prime} \mid\left(x^{\prime}, x^{\prime \prime}\right) \in \Omega\right\}
$$

$\sigma \geqq 0,1 \leqq p \leqq q \leqq \infty, 1 \leqq \xi, \eta \leqq \infty, \varepsilon>0$, and $\lambda=\frac{n}{p}-\frac{m}{q}$.

Then there exist the following trace operators under the additional conditions.

$\mathrm{B}-\mathrm{B} \quad(\mathrm{i}) \quad B_{p, \xi}^{\sigma+\lambda+\varepsilon}(\Omega) \rightarrow B_{q, \eta}^{\sigma}\left(\Omega^{\prime}\right)$,

(ii) $\quad B_{p, \xi}^{\sigma+\lambda}(\Omega) \quad \rightarrow B_{q, \eta}^{\sigma}\left(\Omega^{\prime}\right) \quad(\sigma>0, \xi \leqq \eta)$,

$\mathrm{H}-\mathrm{B}(\mathrm{i}) \quad H_{p}^{\sigma+\lambda+\varepsilon}(\Omega) \rightarrow B_{q, \eta}^{\sigma}\left(\Omega^{\prime}\right)$,

(ii) $\quad H_{p}^{\sigma+\lambda}(\Omega) \quad \rightarrow B_{q, \eta}^{\sigma}\left(\Omega^{\prime}\right) \quad(\sigma>0,1<p \leqq \eta)$,

$\mathrm{B}-\mathrm{H}(\mathrm{i}) \quad B_{p, \xi}^{\sigma+\lambda+\varepsilon}(\Omega) \rightarrow H_{p}^{\sigma}\left(\Omega^{\prime}\right)$,

(ii) $\quad B_{p, \xi}^{\sigma+\lambda}(\Omega) \quad \rightarrow H_{q}^{\sigma}\left(\Omega^{\prime}\right) \quad(\xi \leqq q<\infty, p<q)$,

$\mathrm{H}-\mathrm{H}(\mathrm{i}) \quad H_{p}^{\sigma+\lambda+\varepsilon}(\Omega) \rightarrow H_{q}^{\sigma}\left(\Omega^{\prime}\right)$,

(ii) $H_{p}^{\sigma+\lambda}(\Omega) \rightarrow H_{q}^{\sigma}\left(\Omega^{\prime}\right) \quad(1<p<q<\infty)$.

Proof. Part B-B follows from [20] Theorem 2. Part B-H for the case where $\sigma$ is a non-negative integer also follows from that theorem. Part $\mathrm{B}-\mathrm{H}$ for the case where $\sigma$ is fractional follows from this and the interpolation of operators. Part $\mathrm{H}-\mathrm{B}$ (i) is obtained by

$$
H_{p}^{\sigma+\lambda+\varepsilon}(\Omega) \rightarrow B_{p, \infty}^{\sigma+\lambda+\varepsilon}(\Omega) \rightarrow B_{q, \eta}^{\sigma}\left(\Omega^{\prime}\right)
$$

Part $\mathrm{H}-\mathrm{B}$ (ii). For $f \in H_{p}^{\sigma+\lambda}(\Omega)$ let us use the integral representation given in Theorem 1. By Lemma 4.1 and Lemma 3.3 we see

$$
t^{-\sigma-\lambda} u^{\alpha}, t^{-\sigma-\lambda} u_{\beta}, t^{-\sigma-\lambda} t^{k} f_{\infty}^{(\alpha)} \in L^{p}\left(\Omega ; L_{*}^{\infty}(I)\right)
$$


therefore, in view of Lemma 8.5 , we get

$$
F_{1}\left(x^{\prime}, x^{\prime \prime}\right)+F_{2}\left(x^{\prime}, x^{\prime \prime}\right)+F_{3}\left(x^{\prime}, x^{\prime \prime}\right) \in B_{q, \eta}^{\sigma}\left(\Omega^{\prime}\right) .
$$

Since $F_{4}(x) \in W_{p}^{\infty}, F_{4}\left(x^{\prime}, x^{\prime \prime}\right) \in B_{q, \eta}^{\sigma}\left(\Omega^{\prime}\right)$ is obvious.

Part $\mathrm{H}-\mathrm{H}$ (i) is obtained by considering

$$
H_{p}^{\sigma+\lambda} \mid \varepsilon(\Omega) \rightarrow B_{p, \infty}^{\sigma+\lambda+\varepsilon}(\Omega) \rightarrow H_{q}^{\sigma}\left(\Omega^{\prime}\right)
$$

Part H-H(ii). Case $\sigma=0$. Let $f \in H_{p}^{\lambda}(\Omega)$. Then, in the integral representation given in Theorem 1 ,

$$
t^{-\lambda} u^{\alpha}, t^{-\lambda} u_{\beta}, t^{-\lambda} t^{k} f_{\infty}^{(\alpha)} \in L^{p}\left(\Omega ; L_{\infty}^{2}\right)
$$

Hence by Lemma 8.3 we have

$$
\begin{aligned}
\left\|\int_{0}^{a}\left|\int M_{\alpha}(x,-z) u^{\alpha}(t, x+t z) d z\right| d_{*} t\right\|_{L^{q}\left(\Omega^{\prime}\right)} & \leqq C_{1}\left\|u^{\alpha}\right\|_{L^{p}\left(\Omega ; L_{*}^{2}\right)} \\
& \leqq C_{2}\|f\|_{H_{p}^{\lambda}(\Omega),}
\end{aligned}
$$

which gives

$$
\left\|F_{1}\left(x^{\prime}, x^{\prime \prime}\right)\right\|_{L^{q}\left(\Omega^{\prime}\right)} \leqq C_{3}\|f\|_{H p(\Omega)}^{\lambda} .
$$

The same fact holds for $F_{2}$ and $F_{3}$, and we have shown the assersion for the case $\sigma=0$. For the case where $\sigma$ is a positive integer the result follows from the same argument applied to the derivaties of $f$. Again an application of the interpolation of operators gives the result for the fractional case.

\section{9) Duality}

To discuss duality we shall need a lemma as follows:

Lemma 9.1. Let $\sigma$ be a real number, $i, j$ be positive integrers,

$$
H_{h}(x, z) \in \mathscr{K}_{i}, K_{k}(x, z) \in \mathscr{K}_{0}, L_{l}(x, z) \in \mathscr{K}_{j}, M_{m}(x, z) \in \mathscr{K}_{0},
$$

and $1<p<\infty$.

(I) Assume that $1+\sigma \leqq j, 1-\sigma \leqq i$, and $t^{-\sigma} u_{h} \in L^{p}\left(\Omega ; L_{*}^{2}(I)\right), \quad t^{\sigma} v_{l} \in$ 
$L^{p^{\prime}}\left(\Omega ; L_{*}^{2}(I)\right), \varphi_{k} \in L^{p}(\Omega), \phi_{m} \in L^{p^{\prime}}(\Omega)$, where $p^{\prime}$ is the conjugate exponent of $p$. We define

$$
\begin{array}{r}
\begin{array}{r}
f(x)=\sum_{h} \int_{0}^{a} d_{*} t \int H_{h}(x,-z) u_{h}(t, x+t z) d z \\
\quad+\sum_{k} \int K_{k}(x,-z) \varphi_{k}(x+a z) d z \\
g(x)=\sum_{l} \int_{0}^{a} d_{*} \int L_{l}(x+t z, z) v_{l}(t, x+t z) d z \\
\quad+\sum_{m} \int M_{m}(x+a z, z) \psi_{m}(x+a z) d z
\end{array}
\end{array}
$$

(then $f \in H_{p}^{\sigma}(\Omega)$ and $g \in H_{p^{\prime}, \bar{\Omega}}^{-\sigma}\left(\mathbb{R}^{n}\right)$ ), and set

$$
\begin{aligned}
U_{l}(t, x) & =\int L_{l}(x,-z) f(x+t z) d z, \\
V_{h}(t, x) & =\int H_{h}(x+t z, z) g(x+t z) d z, \\
F_{m}(x) & =\int M_{m}(x,-z) f(x+a z) d z, \\
G_{k}(x) & =\int K_{k}(x+a z, z) g(x+a z) d z .
\end{aligned}
$$

Then $t^{-\sigma} U_{l}, t^{\sigma} V_{h} \in L^{p}\left(\Omega, L_{*}^{2}(I)\right)$, and

$$
\begin{aligned}
& \sum_{l} \int_{0}^{a} d_{*} t \int U_{l}(t, x) v_{l}(t, x) d x+\sum_{m} \int F_{m}(x) \psi_{m}(x) d x \\
= & \sum_{h} \int_{0}^{a} d_{*} t \int u_{h}(t, x) V_{h}(t, x) d x+\sum_{k} \int \varphi_{k}(x) G_{k}(x) d x, \\
= & \lim _{\varepsilon \rightarrow 0} \int f_{\varepsilon}(x) g_{\varepsilon}(x) d x,
\end{aligned}
$$

where $f_{\varepsilon}$ and $g_{\varepsilon}$ are defined by integrating over $\varepsilon \leqq t \leqq a$ in the formulas (9.1) and (9.2), respectively.

(II) Assume that $\sigma<j,-\sigma<i, 1<q<\infty$, and that $t^{-\sigma} u_{h} \in L_{*}^{q}\left(I ; L^{p}(\Omega)\right)$, $t^{\sigma} v_{l} \in L_{*}^{q^{\prime}}\left(I ; L^{p^{\prime}}(\Omega)\right), \varphi_{k} \in L^{p}(\Omega), \phi_{m} \in L^{p^{\prime}}(\Omega)$, where $q^{\prime}$ is the conjugate exponent of $q$. Then, defining $f, g, U_{l}, V_{h}, F_{m}, G_{k}, f_{\varepsilon}$ and $g_{\varepsilon}$ as in Part (I), 
(9.3) holds.

Proof. (l). Let $u_{h, \varepsilon}(t, x)=u_{h}(t, x)$, for $\varepsilon \leqq i \leqq a$, and $u_{h, \varepsilon}(t, x)=0$ otherwise, and let $v_{l, \varepsilon}(t, x)=v_{l}(t, x)$, for $\varepsilon \leqq t \leqq a$, and $v_{l, \varepsilon}(t, x)=0$ otherwise, where $\varepsilon>0$. $U_{l, \varepsilon}, F_{m, \varepsilon}$ and $V_{h, \varepsilon}, G_{k, \varepsilon}$ are the functions defined by $f_{\varepsilon}$ and $g_{\varepsilon}$, respectively. It is known that the dual space of $\mathbb{L}^{p}\left(\Omega ; \mathbb{L}_{*}^{2}(\mathbb{I})\right)$ is the space $L^{p^{\prime}}\left(\mathscr{S} ; L_{*}^{2}(\mathbb{I})\right)$ (cf. [4], [25]), and by the definition of Lebesgue integral we have

$$
\begin{aligned}
& t^{-\sigma} u_{h, \varepsilon} \rightarrow t^{-\sigma} u_{h} \text { in } \mathbb{L}^{p}\left(\Omega ; L_{*}^{2}\right) \quad \text { as } \varepsilon \rightarrow 0, \\
& t^{\sigma} v_{l, \varepsilon} \rightarrow t^{\sigma} v_{l} \quad \text { in } \quad L^{p^{\prime}}\left(\Omega ; L_{*}^{2}\right) \text { as } \varepsilon \rightarrow 0,
\end{aligned}
$$

and there fore by Lemma 4.2

$$
\begin{aligned}
& f_{\varepsilon} \rightarrow f \text { in } H_{p}^{\sigma}(\Omega), \\
& g_{\varepsilon} \rightarrow g \text { in } H_{p^{\prime}}^{-\sigma}\left(\mathbb{F}^{2} n\right),
\end{aligned}
$$

so by Lemma 4.1

$$
\begin{aligned}
& t^{-\sigma} U_{l, \varepsilon} \rightarrow t^{-\sigma} U_{l} \text { in } \quad L^{p}\left(\Omega ; L_{*}^{2}\right), \\
& F_{m, \varepsilon} \rightarrow F_{m} \quad \text { in } \quad L^{p}(\Omega), \\
& t^{\sigma} V_{h, \varepsilon} \rightarrow t^{\sigma} V_{h} \quad \text { in } \quad L^{p^{\prime}}\left(\Omega ; L_{*}^{2}\right), \\
& G_{k, \varepsilon} \rightarrow G_{k} \quad \text { in } \quad L^{p^{\prime}}(\Omega) .
\end{aligned}
$$

Since every term in (9.3) is a continuous bilinear form, it suffices to prove (9.3) for $f_{\varepsilon}$ and $g_{\varepsilon}$.

Now, from Fubini's theorem it follows

$$
\begin{aligned}
& \sum_{l} \int_{0}^{a} d_{* i} i \int U_{l, \varepsilon}(t, x) v_{l, \varepsilon}(t, x) d x+\sum_{m} \int F_{m, \varepsilon}(x) \psi_{m}(x) d x \\
& =\sum_{l} \int_{0}^{a} d_{*} t \iint_{l}(x,-z) f_{\varepsilon}(x+t z) v_{l, \varepsilon}(t, x) d z d x \\
& \quad+\sum_{m} \iint M_{m}(x,-z) f_{\varepsilon}(x+a z) \psi_{m}(x) d z d x
\end{aligned}
$$




$$
\begin{aligned}
= & \int f_{\varepsilon}(y) d y\left\{\sum_{l} \int_{0}^{a} d_{*} t \int L_{l}(y+t z, z) v_{l, \varepsilon}(t, y+t z) d z\right. \\
& \left.+\sum_{m} \int M_{m}(y+a z, z) \phi_{m}(y+a z) d z\right\}, \\
= & \int f_{\varepsilon}(x) g_{\varepsilon}(x) d z,
\end{aligned}
$$

which gives (9.3). The proof of part (II) is analogous.

Combining the lemma with the integral representation (Theorem 1) and Theorem 2, we obtain the following

Corollary。 Let $\sigma$ be a real number and $1<p, q<\infty$.

(I) Define $\omega_{m}(x, z)$ as in Lemma 3.1 Corollary 1 , where $m=i+j-1$, $1+\sigma \leqq j, 1-\sigma \leqq i$, and $j, i$ are positive integers. For $f \in H_{p}^{\sigma}(\Omega), g \in H_{p^{\prime} \bar{\Omega}}^{-\sigma}\left(\mathbb{R}^{n}\right)$ we define

$$
\begin{aligned}
& U_{m}(t, x)=\int \omega_{m}(x,-z) f(x+t z) d z, \\
& V_{m}(t, x)=\int \omega_{m}(x+t z, z) g(x+t z) d z,
\end{aligned}
$$

Then

$$
\lim _{\varepsilon \rightarrow 0} \int U_{m}(\varepsilon, x) V_{m}(\varepsilon, x) d x
$$

converges, and, denoting its value by $\langle f, g\rangle$,

$$
|<f, g>| \leqq C|| f\left\|_{H_{p}^{\sigma}(\Omega)}\right\| g \|_{H_{p^{\prime}}^{-\sigma}\left(\mathbb{R}^{n}\right)} .
$$

(II) For $f \in B_{p, q}^{\sigma}(\Omega), g \in B_{p^{\prime}, q^{\prime}, \bar{\Omega}}^{-\sigma}\left(\mathbb{R}^{n}\right)$ the same facts also hold. But in this case we should take $m=i+j$, where $j>\sigma, i>-\sigma$, and $j, i$ are nonnegative integers.

Hheorem $1 \mathbb{1}$. (Duality). Let $\sigma$ be a real number, and $1<p, q<\infty$. Then

$$
\begin{array}{ll}
{\left[H_{p}^{\sigma}(\Omega)\right]^{\prime}=H_{p^{\prime}, \bar{\Omega}}^{-\sigma}\left(\mathbb{R}^{n}\right),} & {\left[H_{p^{\prime}, \bar{\Omega}}^{-\sigma}\left(\mathbb{R}^{n}\right)\right]^{\prime}=H_{p}^{\sigma}(\Omega),} \\
{\left[B_{p, q}^{\sigma}(\Omega)\right]^{\prime}=B_{p^{\prime}, q^{\prime}, \bar{\Omega}}^{-\sigma}\left(\mathbb{R}^{n}\right),} & {\left[B_{p^{\prime}, q^{\prime}, \bar{\Omega}}^{-\sigma}\left(\mathbb{R}^{n}\right)\right]^{\prime}=B_{p, q}^{\sigma}(\Omega),}
\end{array}
$$


where prime denotes the dual space, $p^{\prime}$ and $q^{\prime}$ are conjugate exponent of $p$ and $q$, respectively.

In more detail, the duality is given by the bilinear form

$$
<f, g>=\lim _{\varepsilon \rightarrow 0} \int U_{m}(\varepsilon, x) V_{m}(\varepsilon, x) d x
$$

Here $U_{m}$ and $V_{m}$ are defined by (9.4) and (9.5), respectively.

Proof. (I). Let $j$ and $i$ be positive integers such that $1+\sigma \leqq j$, $1-\sigma \leqq i$. We shall make use of the integral representation (3.5), in which we take $l=m=i+j-1, k=j-1, h=i-1$. By the above corollary we have known that $\langle f, g\rangle$ is a continuous bilinear form on $H_{p}^{\sigma}(\Omega) \times H_{p^{\prime}, \bar{\Omega}}^{-\sigma}\left(\mathbb{R}^{n}\right)$.

To prove that $\langle f, g\rangle=0$ for every $g \in H_{p^{\prime}, \bar{\Omega}}^{-\sigma}\left(\mathbb{R}^{n}\right)$ implies $f=0$, let us take $\tilde{V}(t, x) \in L^{p^{\prime}}\left(\Omega ; L_{*}^{2}(I)\right)$. For $|\beta| \leqq h$ define

$$
\tilde{v}^{\beta}(t, x)=\int_{t}^{a} t^{h} s^{-|\beta|-\sigma} \tilde{V}(s, x) d_{*} s
$$

Then $t^{\sigma} \widetilde{v}^{\beta} \in L^{p^{\prime}}\left(\Omega ; L_{*}^{2}\right)$, so that

$$
g(x)=\int_{0}^{a} d_{*} t L_{\beta}(x+t z, z) \tilde{v}^{\beta}(t, x+t z) d z \in H_{\bar{p}^{\prime}, \bar{\Omega}}^{-\sigma}
$$

Hence, by Lemma 9.1 and Fubini's theorem we have

$$
\begin{aligned}
<f, g> & =\int_{0}^{a} d_{* t} \int U_{\beta}(t, x) \tilde{v}^{\beta}(t, x) d x \\
& =\int_{0}^{a} d_{*} t \int_{t}^{a} d_{*} s \int t^{h} s^{-|\beta|-\sigma} \tilde{V}(s, x) U_{\beta}(t, x) d x \\
& =\int_{0}^{a} d_{*} t \int t^{-\sigma+h-|\beta|} u_{\beta}(t, x) \tilde{V}(t, x) d x
\end{aligned}
$$

Thus, $\langle f, g\rangle=0$ for every $g \in H_{p, \bar{\Omega}}^{-\sigma}$ implies that the right hand side is equal to zero for every $\tilde{V} \in L^{p^{\prime}}\left(\Omega ; L_{*}^{2}\right)=\left[L^{p}\left(\Omega ; L_{*}^{2}\right)\right]^{\prime}$, which gives $u_{\beta}(t$, $x)=0$ for every $|\beta| \leqq h$, so that $F_{2}(x)=0$. Analogously we can obtain $F_{1}(x)=0$. Also, substituting

$$
g(x)=\int \omega_{m}(x+a z, z) \tilde{G}(x+a z) d z
$$


where $\tilde{G}$ is an arbitrary function in $L^{p^{\prime}}(\Omega)$, we get $f_{\infty}(x)=0$, and, hence, $F_{3}(x)=F_{4}(x)=0$. Thus we have $f(x)=0$.

Likewise, we can show that $\langle f, g\rangle=0$ for every $f \in H_{p}^{\sigma}$ implies $g=0$.

From the observations we have done it follows that $H_{p}^{\sigma}(\Omega) \subset\left[H_{p^{\prime}}^{-\sigma}, \bar{\Omega}\left(\mathbb{R}^{n}\right)\right]^{\prime}$, and $H_{p^{\prime}}^{-\sigma}, \bar{\Omega}\left(\mathbb{R}^{n}\right) \subset\left[H_{p}^{\sigma}(\Omega)\right]^{\prime}$. Thus the theorem is established if the converse inclusion is shown.

To do this, let $F \in\left[H_{p}^{\sigma}(\Omega)\right]^{\prime}$. Since for any $u \in L^{p}\left(\Omega ; L_{*}^{2}\right)$

$$
\int_{0}^{a} d_{*} t \int M_{\alpha}(x,-z) t^{\sigma} u(t, x+t z) d z \in H_{p}^{\sigma}
$$

the functional

$$
F\left(\int_{0}^{a} d_{*} t \int M_{\alpha}(x,-z) t^{\sigma} u(t, x+t z) d z\right)
$$

is continuous on $L^{p}\left(\Omega ; L_{*}^{2}\right)$, and, hence, this is equal to

$$
\int_{0}^{a} d_{*} t \int u(t, x) \tilde{V}_{\alpha}(t, x) d x
$$

for some $\tilde{V}_{\alpha}(t, x) \in L^{p^{\prime}}\left(\Omega ; L_{*}^{2}\right)$. Similarly, there exist $\tilde{V}^{\beta}(t, x) \in L^{p^{\prime}}\left(\Omega ; L_{*}^{2}\right)$, $\tilde{G}^{\beta} \in L^{p^{\prime}}(\Omega)$ such that

$$
F\left(\int_{0}^{a} d_{*} t \int M^{(0, \beta)}(x,-z) t^{\sigma} u(t, x+t z) d z\right)=\int_{0}^{a} d_{*} t \int u(t, x) \tilde{V}^{\beta}(t, x) d x,
$$

for every $u(\iota, x) \in L^{p}\left(\Omega ; L_{*}^{2}\right)$, and

$$
F\left(\int a^{-|\beta|} \omega_{m}{ }^{(0, \beta)}(x,-z) \varphi(x+a z) d z\right)=\int \varphi(x) \tilde{G}^{\beta}(x) d x
$$

for every $\varphi \in L^{p}(\Omega)$. Put

$$
\begin{gathered}
\tilde{v}_{\alpha}(t, x)=\int_{0}^{t} s^{-\sigma+k} t^{-k} \tilde{V}_{\alpha}^{i}(s, x) d_{*} s, \\
\tilde{v}^{\beta}(t, x)=\int_{t}^{a} s^{-\sigma-|\beta|} t^{h} \tilde{V}^{\beta}(s, x) d_{*} s, \\
\tilde{g}_{\alpha}(x)=\int_{0}^{a} t^{k-\sigma} \tilde{V}_{\alpha}(t, x) d_{*} t,
\end{gathered}
$$

and set 


$$
\begin{aligned}
g(x)= & \sum_{|\beta| \leqq h} \int_{0}^{a} d_{*} t \int L_{\beta}(x+t z, z) \tilde{v}^{\beta}(t, x+t z) d z \\
& +\sum_{|\alpha|=k} \int_{0}^{a} d_{*} t \int D_{z}^{\alpha}\{L(x+t z, z)\} \tilde{v}_{\alpha}(t, x+t z) d z \\
& +\sum_{|\alpha|=k} \sum_{\beta}\left(\begin{array}{l}
\alpha \\
\beta
\end{array}\right) \int a^{-|\beta|} \omega_{m}^{(\alpha-\beta, \beta)}(x+a z, z) \tilde{g}_{\alpha}(x+a z) d z \\
& +\sum_{|\beta| \leqq h} \int_{0}^{a} d_{*} t \int L_{\beta}(x+t z, z) t^{h} \tilde{G}^{\beta}(x+t z) d z \\
& +\int(\omega){ }_{m}(x+a z, \tilde{z}) \tilde{G}^{0}(x+a z) d z^{\circ}
\end{aligned}
$$

Then, by Lemma 9.1 we obtain, for any $f \in H_{p}^{\circ}(\Omega)$,

$$
\begin{aligned}
& \langle f, g\rangle=\sum_{|\beta| \leqq h} \int_{0}^{a} d_{*} t \int U_{\beta}(t, x) \tilde{v}^{\beta}(t, x) d x \\
& +\sum_{|\alpha|=k} \int_{0}^{a} d_{*} t \int U^{\alpha}(t, x) \tilde{v}_{\alpha}(t, x) d x \\
& +\sum_{\mid \alpha_{1}=k} \int f_{\infty}^{(\alpha)}(x) \tilde{g}_{\alpha}(x) d x \\
& +\int\left\{\sum_{|\beta| \leqq h} f_{\beta}(x) \tilde{G}^{\beta}(x)+f_{\infty}(x) \tilde{G}^{0}(x)\right\} d x \\
& =\sum_{|\beta| \leqq h} \int_{0}^{a} d_{*} s \int s^{h-|\beta|-\sigma} u_{\beta}(s, x) \tilde{V}^{\beta}(s, x) d x \\
& +\sum_{|\alpha|=k} \int_{0}^{a} d_{*} s \int s^{-\sigma} u^{\alpha}(s, x) \tilde{V}_{\alpha}(s, x) d x \\
& +\sum_{|\alpha|=k} \int_{0}^{a} d_{*} s \int s^{k-\sigma} f_{\infty}^{(\alpha)}(x) \tilde{V}_{\alpha}(s, x) d x \\
& +\int\left\{\sum_{|\beta| \leqq h} f_{\beta}(x) \tilde{G}^{\beta}(x)+f_{\infty}(x) \tilde{G}^{0}(x)\right\} d x \\
& =F\left(\sum_{|\alpha|=k} \int_{0}^{a} d_{*} s \int M_{\alpha}(x,-z) u^{\alpha}(s, x+s z) d z\right. \\
& +\sum_{|\beta| \leqq h} \int_{0}^{a} d_{*} s \int s^{h-|\beta|} M^{(0, \beta)}(x,-z) u_{\beta}(s, x+s z) d z
\end{aligned}
$$




$$
\begin{aligned}
& +\sum_{|\alpha|=k} \int_{0}^{a} d_{*} s \int M_{\alpha}(x,-z) s^{k} f_{\infty}^{(\alpha)}(x+s z) d z \\
& +\sum_{|\beta| \leqq h} \int^{-|\beta|} \omega_{m}^{(0, \beta)}(x,-z) f_{\beta}(x+a z) d z \\
& \left.+\int \omega_{m}(x,-z) f_{\infty}(x+a z) d z\right) \\
& =F(f),
\end{aligned}
$$

in view of Theorem 1, and the inclusion $\left(H_{p}^{\sigma}\right)^{\prime} \subset H_{p^{\prime}, \bar{\Omega}}^{-\sigma}$ is established.

By the same argument, we find that for $G \in\left(H_{p^{\prime}, \bar{\Omega}}^{-\sigma}\right)^{\prime}$ there exist $\tilde{U}_{\beta}, \tilde{U}^{\alpha} \in L^{p}\left(\Omega ; L_{*}^{2}\right), \tilde{F}^{0}, \tilde{F}^{\alpha} \in L^{p}(\Omega)$ such that

$$
\begin{aligned}
& G\left(\int_{0}^{a} d_{*} t \int L_{\beta}(x+t z, z) t^{-\sigma} v(t, x+t z) d z\right)=\int_{0}^{a} d_{*} t \int v(t, x) \tilde{U}_{\beta}(t, x) d x, \\
& G\left(\int_{0}^{a} d_{*} t \int D_{z}^{\alpha}\{L(x+t z, z)\} t^{-\sigma} v(t, x+t z) d z\right)=\int_{0}^{a} d_{*} t \int v(t, x) \tilde{U}^{\alpha}(t, x) d x, \\
& G\left(\int^{-|\alpha|} D_{z}^{\alpha}\left\{\omega_{m}(x+a z, z)\right\} \phi(x+a z) d z\right)=\int \phi(x) \tilde{F}^{\alpha}(x) d x
\end{aligned}
$$

for any $v \in \mathbb{L}^{p^{\prime}}\left(\Omega ; \mathbb{L}_{*}^{2}\right), \psi \in \mathbb{L}^{p^{\prime}}(\Omega)$, and, putting

$$
\begin{aligned}
f(x)= & \sum_{|\alpha|=k} \int_{0}^{a} d_{*} t \int M_{\alpha}(x,-z)\left\{\iint_{t}^{a} t^{k} s^{\sigma-k} \tilde{U}^{\alpha}(s, x+t z) d_{*} s\right\} d z \\
& +\sum_{|\beta| \leqq h} \int_{0}^{a} d_{*} t \int t^{h-|\beta|} M^{(0, \beta)}(x,-z)\left\{\int_{0}^{t} t^{-h} s^{h+\sigma} \widetilde{U}_{\beta}(s, x+t z) d_{*} s\right\} d z \\
& +\sum_{|\alpha|=k} \int_{0}^{a} d_{* t} \int M_{\alpha}(x,-z) t^{k} \tilde{F}^{\alpha}(x+t z) d z \\
& +\sum_{|\beta| \leqq h} \int^{-|\beta|} \omega_{m}^{(0, \beta)}(x,-z)\left\{\int_{0}^{a} t^{h+\sigma} \widetilde{U}_{\beta}(t, x+a z) d_{*} t\right\} d z \\
& +\int \omega_{m}(x,-z) \tilde{F}^{0}(x+a z) d z
\end{aligned}
$$

by Lemma 9.1 and Theorem 1 we obtain

$$
<f, g>=G(g)
$$

for any $g \in H_{p^{\prime}, \bar{\Omega}}^{-\sigma}\left(\mathbb{R}^{n}\right)$. 
This completes the proof of part (I).

The proof of part (II) is the same, except that we should remark

$$
\left[L_{*}^{q}\left(I ; L^{p}(\Omega)\right)\right]^{\prime}=L_{*}^{q^{\prime}}\left(I ; L^{p^{\prime}}(\Omega)\right) .
$$

\section{References}

[1] Adams, R., Aronszajn, N. and Smith, K.T., Theory of Bessel potentials. Part II. Ann. Inst. Fourier (Grenoble), 17 (1967), 1-135.

[2] Aronszajn, N. and Smith, K.T., Theory of Bessel potentials I. Ann. Inst. Fourier (Grenoble), 11 (1961), 385-475.

[3] Benedek, A., Calderón, A.P. and Panzone, R., Convolution operators on Banach space valued functions. Proc. Nat. Acd. Sci. U.S.A., 48 (1962), 356-365.

[4] Benedek, A. and Panzone, R., The spaces $\mathrm{L}^{\mathrm{p}}$ with mixed norm, Duke Math. J. 28 (1961), 301-324.

[5] Besov, O.V., On a certain family of functional spaces, imbedding and continuation, Dokl. Akad. Nauk SSSR, 126 (1959), 1163-1165. (Russian).

[6] - Investigation of a family of function spaces in connection with theorems of imbedding and extension, Trudy Mat. Inst. Steklov, 60 (1961), 42-81.

[7] Calderón, A.P., Lebesgue spaces of differentiable functions and distributions. Proc. Symp. in Pure Math. 5 (1961), 33-49.

[8] - Intermediate spaces and interpolation, the complex method. Studia Math. 24 (1964), 113-190.

[9] Gagliardo, E., Caratterizzazioni delle traces sulla frontiera ad alcune classi di funzioni in $n$ variabili. Rend. Sem. Mat. Univ. Padova, 27 (1957), 284-305.

[10] — Proprietà di alcune classi di funzioni in più variabili. Richerche di Mat. Napoli, 7 (1958), 102-137.

[11] Hardy, G.H. and Littlewood, J.E., Theorems concerning mean values of analytic or harmonic functions. Quart. J. Math. Oxford Ser., 12 (1942), 221-256.

[12] Komatsu, H., Fractional powers of operators. Pacific J. Math., 19 (1966), 285-346. II. ibid. 21 (1967), 89-111. III. J. Math. Soc. Japan, 21 (1969), 205-220. V. J. Fac. Sci. Univ. Tokyo, Sec. IA, 17 (1970), 373-396. VI. ibid. 19 (1972), 1-63.

[13] John, F. and Nirenberg, L., On functions of bounded mean oscillation, Comm. Pure Appl. Math. 14 (1961), 415-426.

[14] Lax, P.D., On Cauchy's problem for hyperbolic equations and the differetiability of solution of elliptic equations. Comm. Pure Appl. Math. \& (1955), 615-633.

[15] Lions, J.L., Une construction d'espaces d'interpolation. C.R. Acad. Sci. Paris Sér. A-B. 251 (1960), 1853-1855.

[16] Lions, J. L. et Magenes, E., Problemi ai limiti non omogenei, III, IV, Ann. Scuola Norm. Sup. Pisa, 15 (1961), 41-103, 311-326.

[17] Lions, J. L. et Peetre, J., Sur une classe d'esapces d'interpolation, Publ. Math. IHES, 19 (1964), 5-68.

[18] Muramatu, T., On Besov spaces of functions defined in general regions. Publ. RIMS, Kyoto Univ., 6 (1970), 515-543.

[19] — Products of fractional powers of operators. J. Fac. Sci. Univ. Tokyo, Sec. IA, 17 (1970), 581-590. 
[20] - On imbedding theorems for Besov spaces of functions defined in general regions. Publ. RIMS, Kyoto Univ., 7 (1971), 261-285.

[21] — On the boundedness of a class of operator-valued pseudo-differential operators in $\mathrm{L}^{\mathrm{p}}$-space, Proc Japan Acad. 4.9 1973, 94-99.

[22] Nikol'skii, S.M., On the imbedding, continuation and approximation theorems for differentiable functions in several variables, Uspehi Mat. Nauk, 16 (1961), 63114. Russian Math. Surveys, 16 (1961), 55-104.

[23] Nikolsky, S.M., Lions, J. and Lizorkin, L.I., Integral representation and isomorphism properties of some classes of functions, Ann. Scuola Norm. Sup. Pisa, 19 (1965), 127-178.

[24] Nirenberg, L., On elliptic partial differential equations, Ann. Scuola Norm. Sup. Pisa, 13 (1959), 115-161.

[25] Phillips, R. S., On weakly compart subsets of a Banach space. Amer. J. Math. 65 (1943), 108-136.

[26] Schwartz, L., Theorie des distributions à valeus vectorielles I. Amn. Inst. Fourier (Grenoble), 7 (1957), 1-139. II. ibid. \& (1958), 1-209.

[27] Schechter, M., Complex interpolation. Composito Math. 18 (1967), 117-147.

[28] Sobolev, S.L., On a theorem in functional analysis. Mat. Sb. (1938), 471-497. (Russian).

[29] Stein, E.M., On the functions of Littlewood-Paley, Lusin and Marcinkiewicz, Trans. Amer. Math. Soc. 88 (1958), 430-466.

[30] $\longrightarrow$, On some functions of Littlewood-Paley and Zygmund, Bull. Amer. Math. Soc., 67 (1961), 99-101.

[31] Singular Integrals and differentiability properties of functions. Princeton Univ. Press. 1970.

[32] Taibleson, M. H., On the theory of Lipschitz spaces of distributions on Euclidean n-space I. J. Math. Mech. 13 (1964), 407-479. II. ibid. 14 (1965), 821-839. III. ibid. 15 (1966), 973-981.

[33] Yoshikawa, A., Fractional powers of operators, interpolation theory and imbedding theorems, J. Fac. Sci. Univ. Tokyo Sec. IA, 18 (1971), 335-362.

[34] Yosida, K., Functional Analysis. Springer Verlag, (1966). 